\title{
An SPDE Model for Systemic Risk with Endogenous Contagion
}

\author{
Ben Hambly and Andreas Søjmark \\ Mathematical Institute, University of Oxford
}

September 28, 2018

\begin{abstract}
We propose a dynamic mean field model for 'systemic risk' in large financial systems, which we derive from a system of interacting diffusions on the positive half-line with an absorbing boundary at the origin. These diffusions represent the distancesto-default of financial institutions and absorption at zero corresponds to default. As a way of modelling correlated exposures and herd behaviour, we consider a common source of noise and a form of mean-reversion in the drift. Moreover, we introduce an endogenous contagion mechanism whereby the default of one institution can cause a drop in the distances-to-default of the other institutions. In this way, we aim to capture key 'system-wide' effects on risk. The resulting mean field limit is characterized uniquely by a nonlinear SPDE on the half-line with a Dirichlet boundary condition. The density of this SPDE gives the conditional law of a non-standard 'conditional' McKean-Vlasov diffusion, for which we provide a novel upper Dirichlet heat kernel type estimate that is essential to the proofs. Depending on the realizations of the common noise and the rate of mean reversion, the SPDE can exhibit rapid accelerations in the loss of mass at the boundary. In other words, the contagion mechanism can give rise to periods of significant systemic default clustering.
\end{abstract}

\section{Introduction}

One of the most important lessons of the 2007-2009 financial crisis is the imperative nature of system-wide perspectives on risk. That is, financial models need to take into account the interconnectedness of the financial system and they must incorporate sensible notions of financial contagion whereby the distress of one institution can lead to losses for the other members of the system. While such ideas have already had implications for macroprudential policies (Benoit et al. [5], Duffie [22]) and stress testing practices (Dees, Henry \& Martin [19]), there is still a pressing need for a better understanding of the dynamic feedback effects and amplification mechanisms that seem to have been the real drivers of the financial crisis.

Indeed, it is widely accepted that — up to an order of magnitude - the extent of the crisis cannot be explained by simple references to (linear) exogenous shocks such as the devaluation of mortgage-backed securities (Cochrane [13], Brunnermeier [7, Hellwig [37]). Instead, smaller scale shocks are understood to have unfolded into a spiral of events rooted within the financial system itself and amplified by a myriad of interactions between the 
individual institutions. Accordingly, there have been judicious calls for a better understanding of the endogenous (nonlinear) nature of systemic risk (Pedersen [47, Danielsson, Shin \& Zigrand [18]) and it has been emphasized that systemic risk is inherently dynamic with a gradual build-up typically taking place in the background before it materializes in a crisis (Brunnermeier, Gorton \& Krishnamurthy [8]).

\subsection{A system-wide perspective on risk}

Consider a 'representative' member of a large financial system and let $X_{t} \in[0, \infty)$ denote a measure of its financial health at time $t$, which we call its distance-to-default. Neglecting system-wide effects, it is a classical approach in structural credit risk theory to simply model $X_{t}$ in isolation by a Brownian motion with drift - where default occurs at the first hitting time of zero. If 'isolation' is taken to mean that all the actors are assumed independent, then this means that the overall health of the financial system is effectively described by a linear (deterministic) heat equation.

The aim of this paper is to introduce systemic risk into this picture by instead proposing a mean field model derived from an interacting particle system that incorporates simple notions of (i) common exposures, (ii) herd behaviour, and (iii) endogenous contagion. In turn, the health of the financial system will now be described by a nonlinear mean field type SPDE on the positive half-line (Theorems $2.4 \& 2.6$ ) and, in a suitable sense, the dynamics of a 'representative' financial institution is no longer a Brownian motion with drift but rather a conditional McKean-Vlasov type SDE with dependence on the conditional law of its paths given the noise of the common exposures (Theorem 2.7).

\subsection{Established literature on systemic risk}

A decade after the global financial crisis, there is by now a significant body of work dealing with the complex web of interactions in the financial system and the dual rôle of interconnectedness as a source of diversification or a channel for contagion.

In terms of mathematical modelling, it is possible to identify three main approaches to the challenges of systemic risk. First, there is the large literature on network-based models for clearing and contagion, which extend the early frameworks of Eisenberg \& Noe [24] and Allen \& Gale [2] (for a comprehensive review of this approach, see 32]). While these network models are principally static, a dynamic extension has recently been studied in Banerjee, Bernstein \& Feinstein [4. Next, there is the much smaller literature on dynamic mean field models in the spirit of Carmona, Fouque \& Sun [11] and Garnier, Papanicolaou \& Wei [27]. These models benefit from a richer dynamic and stochastic structure, but they tend to focus on quite simple interbank interactions that neglect defaults and contagion (see also [25, 26, 10, 28]). Lastly, there is the related reduced-form literature on intensitybased models for large portfolio credit risk (see e.g. [30, 31, [17]). These models seek to incorporate implicit notions of default contagion and they have been discussed in a systemic risk context by Spiliopoulos [50] and Giesecke, Schwenkler \& Sirignano [29].

The model we propose here belongs naturally to the mean field literature, however, we develop a more flexible framework that incorporates contagion endogenously via a structural mechanism for defaults. This approach differs markedly from the reduced-form literature, where contagion is in the form of self-exciting point processes, and it has the added benefit of being conceptually close to the network-based approaches. 


\subsection{The endogenous contagion mechanism}

Our starting point is inspired by recent dynamic frameworks for the structural modelling of large portfolio credit risk (see e.g. [35, 9]). Specifically, we identify each financial institution (henceforth: bank) with a notion of its distance-to-default given by

$$
Y_{t}^{i}=\log \left(A_{t}^{i}\right)-\log \left(D_{t}^{i}\right), \quad \text { for } i=1, \ldots, N,
$$

where $A_{t}^{i}$ is the market value of bank $i$ 's assets and $D_{t}^{i}$ denotes its default barrier. These distances-to-default will be modelled by suitable stochastic processes on $(0, \infty)$ with absorption at the origin corresponding to default. The precise dynamics will be specified in Section 1.4, but first we discuss how to incorporate the contagion mechanism.

For simplicity, we assume that the system can be described by assigning a weight $a_{i}^{N}=a_{i} / \sum_{n=1}^{N} a_{n}$ to each of the banks, where $c \leq a_{i} \leq C$ for fixed constants $C, c>0$. These weights can depend on the initial distances-to-default, and they reflect the relative importance of the banks in the sense that $a_{i}^{N}$ will determine the strength of bank $i$ 's impact on the others. Notice that $\sum_{i=1}^{N} a_{i}^{N}=1$ with $a_{i}^{N}=O(1 / N)$ as $N \rightarrow \infty$. In particular, no single bank can have a macroscopic effect on the system in the large population limit.

Remark 1.1 (Systemically important banks). Since our model will contain a common source of noise, the latter condition is not as restrictive as it may appear. Indeed, we could model a group of particularly influential banks by a separate diffusion and then treat this as a common input in the dynamics of the smaller banks.

Suppose bank $j$ is the first to default. Its contagious impact on any other bank $i$ will be determined by the weight $a_{j}^{N}$ and a parameter $\alpha_{t}^{i} \geq 0$ measuring how costly defaults are to bank $i$ at time $t$. Specifically, we model the resulting contagion by 'discounting' the asset values of the other banks according to the rule

$$
A_{.}^{i} \longmapsto \hat{A}^{i}:=\exp \left\{-a_{j}^{N} \int_{0}^{\cdot} \alpha_{s}^{i} d \mathfrak{L}_{s}^{j, N}\right\} A_{.}^{i}, \quad \text { for each } i \neq j,
$$

with $\mathfrak{L}_{t}^{j, N}:=\int_{0}^{t} \mathfrak{K}(t-r) \mathbf{1}_{r \geq \tau_{j}} d r$, where the impact kernel $\mathfrak{K} \in L^{1}\left(\mathbb{R}_{+}\right)$models the gradual realisation of the losses spurred by the default. We stress that these losses are not restricted to direct counterparty exposures, but may also arise from more indirect sources such as emerging liquidity shortages, fire sales, and drops in confidence. Observe that $\mathfrak{L}_{t}^{j, N}=0$ for $t \leq \tau_{j}$ and, by requiring that $\|\mathfrak{K}\|_{L^{1}}=1$, we have $\mathfrak{L}_{t}^{j, N}=1$ for all $t \geq \tau_{j}+\varepsilon$ whenever $\operatorname{supp} \mathfrak{K} \subseteq[0, \varepsilon]$, for some $\varepsilon>0$.

Remark 1.2 (Interpretations of $\alpha$ ). Consider the case where $\alpha$ is a fixed constant and $\operatorname{supp} \mathfrak{K} \subseteq[0, \varepsilon]$. At time $t=\tau_{j}+\varepsilon$, the discounting in 1.1 is then of the form

$$
\hat{A}_{t}^{i}=\exp \left\{-\alpha a_{j}^{N}\right\} A_{t}^{i} \simeq\left(1-\alpha a_{j}^{N}\right) A_{t}^{i},
$$

for large $N$, since $a_{j}^{N}=O(1 / N)$. In other words, by time $\tau_{j}+\varepsilon$, the default of bank $j$ has caused each bank $i \neq j$ to lose a proportion $\alpha a_{j}^{N}$ of their asset values, relative to what they would have been worth without the contagion. Note also that $\alpha$ can be related to the connectivity of the system: Suppose, for example, that each default only affects a randomly sampled proportion $\hat{p}$ of the banks, each losing $\alpha a_{j}^{N}$ times their asset values upon bank $j$ 's default. As $N$ gets large, this has a similar effect on the system as all the banks incurring the smaller loss of $\hat{\alpha} a_{j}^{N}$ times their asset values, where $\hat{\alpha}:=\hat{p} \alpha$. 
As more banks default, we continue to apply the rule from (1.1). Therefore, the actual (updated) asset values, $\hat{A}$, are given by

$$
\hat{A}_{t}^{i}:=\prod_{j \neq i} \exp \left\{-a_{j}^{N} \int_{0}^{t} \alpha_{s}^{i} d \mathfrak{L}_{s}^{j, N}\right\} A_{t}^{i}=\exp \left\{-\sum_{j \neq i} a_{j}^{N} \int_{0}^{t} \alpha_{s}^{i} d \mathfrak{L}_{s}^{j, N}\right\} A_{t}^{i},
$$

for $i=1, \ldots, N$, where $\mathfrak{L}_{t}^{j, N}:=\int_{0}^{t} \mathfrak{K}(t-r) \mathbf{1}_{r \geq \hat{\tau}_{j}} d r$ with $\hat{\tau}_{j}:=\inf \left\{t>0: \hat{Y}_{t}^{j} \leq 0\right\}$ and $\hat{Y}_{t}^{j}:=\log \left(\hat{A}_{t}^{j}\right)-\log \left(D_{t}^{j}\right)$. Summing over the terms $a_{j}^{N} \mathfrak{L}_{s}^{j, N}$, this simplifies to

$$
\hat{A}_{t}^{i}=\exp \left\{-\int_{0}^{t} \alpha_{s}^{i} d \mathfrak{L}_{s}^{N}\right\} A_{t}^{i}, \quad \text { for } \quad t<\hat{\tau}_{i}, \quad i=1, \ldots, N,
$$

where

$$
\mathfrak{L}_{t}^{N}:=\int_{0}^{t} \mathfrak{K}(t-s) L_{s}^{N} d s \quad \text { and } \quad L_{t}^{N}:=\sum_{j=1}^{N} a_{j}^{N} \mathbf{1}_{t \geq \hat{\tau}_{j}} .
$$

Taking logarithms in 1.2, it follows that the actual (updated) distances-to-default, $\hat{Y}$, have dynamics of the form

$$
d \hat{Y}_{t}^{i}=d Y_{t}^{i}-\alpha_{t}^{i} d \mathfrak{L}_{t}^{N} \quad \text { for } \quad t<\hat{\tau}_{i}, \quad i=1, \ldots, N .
$$

Here the first part, $Y_{t}^{i}$, is simply the original distance-to-default without contagion, while the latter part is a new contagion term driven by the contagion process, $\mathfrak{L}_{t}^{N}$, from $(1.3)$.

Remark 1.3 (The impact kernel $\mathfrak{K}$ ). Further to the above, we assume $\mathfrak{K} \in \mathcal{W}_{0}^{1,1}\left(\mathbb{R}_{+}\right.$), with $\|\mathfrak{K}\|_{1}=1$, where $\mathcal{W}_{0}^{1, p}\left(\mathbb{R}_{+}\right)$denotes the Sobolev space with one weak derivative in $L^{p}$ and zero trace. The benefits of this construction are: $\mathfrak{L}_{t}^{N}$ remains adapted, it inherits the monotonicity of $L_{t}^{N}$, and it has a weak derivative $\mathfrak{K}^{\prime} * L^{N} \in L^{\infty}$. The rôle of the kernel is to impose a continuous notion of latency whereby the impact of contagion is realised gradually as counterparty exposures are sorted out and indirect effects start to kick in.

Remark 1.4 (Capital structure). As may be expected, the default contagion alone cannot deplete the entire asset base. However, due in large part to the low volatility of banking assets in normal times, financial institutions tend to have leverage ratios as high as $85-95 \%$ (see e.g. [6, 33]). Thus, there is ample room for the contagion to be detrimental.

\subsection{A simple model for systemic risk}

In addition to contagion, we want our model to include common exposures and a notion of herding. The two latter effects have already been considered in Carmona, Fouque \& Sun [11], by means of a (constant-coefficient) particle system with a common Brownian motion and mean reversion in the drift. Inspired by this, we can now present a precise formulation of our 'base case' model for systemic risk: Letting $X^{j}$ denote the actual distances-to-default with default times $\tau_{j}:=\inf \left\{t>0: X_{t}^{j} \leq 0\right\}$, for $j=1, \ldots, N$, we propose to model a large financial system by an interacting particle system of the form

$$
\begin{aligned}
d X_{t}^{j}= & \mu\left(t, X_{t}^{j}\right) d t+\pi\left(t, \nu_{t}^{N}\right) \sum_{i=1}^{N} a_{i}^{N} \cdot\left\{\left(X_{t}^{i} \mathbf{1}_{t<\tau_{i}}+\gamma\left(t, \nu_{t}^{N}\right) \mathbf{1}_{t \geq \tau_{i}}\right)-X_{t}^{j}\right\} d t \\
& +\sigma\left(t, X_{t}^{j}\right)\left(\sqrt{1-\rho\left(t, \nu_{t}^{N}\right)^{2}} d W_{t}^{j}+\rho\left(t, \nu_{t}^{N}\right) d W_{t}^{0}\right)-\alpha\left(t, X_{t}^{j}, \nu_{t}^{N}\right) d \mathfrak{L}_{t}^{N},
\end{aligned}
$$


where $W^{0}$ and $W^{1}, \ldots, W^{N}$ are independent Brownian motions with

$$
\mathfrak{L}_{t}^{N}=\int_{0}^{t} \mathfrak{K}(t-s) L_{s}^{N} d s, \quad L_{t}^{N}=\sum_{i=1}^{N} a_{i}^{N} \mathbf{1}_{t \geq \tau_{i}}, \quad \text { and } \quad \nu_{t}^{N}=\sum_{i=1}^{N} a_{i}^{N} \mathbf{1}_{t<\tau_{i}} \delta_{X_{t}^{i}} .
$$

Notice that, after each default, we do not renormalize the mean reverting interaction in the drift as it is intended to reflect herding in investment decisions: if banks are defaulting, this suggests the investments are not performing and thus defaults should not suddenly adjust the drift upwards by a renormalization. Also, we emphasize that our model is intended for the study of short-run market imperfections rather than the long-run behaviour of the financial system. The parameters can be summarized as follows:

- $\sigma$ and $\mu$ model, respectively, the volatility of the banks and their core return net of the rate of change in the default barrier.

- $\rho$ is the correlation parameter which reflects the extent of common exposures.

- $\alpha$ is the contagion parameter which decides how costly defaults are to the system.

- $\pi$ determines the rate of mean reversion which adjusts the core return due to herding in investment decisions or other interbank interactions.

- $\gamma$ can capture what is left after defaults and could be influenced by actions of the central bank or government seeking to stabilize the drift of the system.

In order to capture system-wide influences on these variables, it is natural to allow them to depend on the empirical measure $\nu^{N}$. In particular, the correlation can then act as an indirect source of contagion, in line with the observation that correlations tend to increase in times of financial distress (see e.g. Cont \& Wagalath [16, 15]). Similarly, the rate of herding and the costliness of contagion can vary with the health of the system, which can capture potentially self-reinforcing amplification mechanisms. Furthermore, we will incorporate discontinuities in the dependence on the losses, $L_{t}^{N}$, which can allow for more abrupt adjustments of the herding, the correlation, or the costliness of defaults (see also Section 2.3.2.

\subsection{Outline of the paper}

In Section 2 we state our main results concerning the existence of a unique mean field limit for a general version of the systemic risk model from Section 1.4. Furthermore, we discuss some qualitative insights for systemic risk and consider closely related problems.

In Section 3 we study the regularity of the particle system, which is centred around the boundary and tail behaviour of the densities of the particles. The backbone of this is a novel family of upper Dirichlet heat kernel type estimates, whose proofs we postpone to Section 6 in order to make the presentation as clear as possible.

In Section 4 we proceed to establish tightness of the system and we show that the resulting limit points are solutions to a nonlinear SPDE on the positive half-line.

In Section 5 we rely on energy estimates to prove uniqueness of the SPDE and thus we deduce the full convergence in law to this limit. We present the uniqueness proof in Section 5.1, postponing the technical estimates to Sections 5.2 and 5.3. Therefore, the reader can get a complete picture of existence and uniqueness by only reading up to Section 5.1. 


\section{Main results}

To make our framework as flexible as possible, we will consider a more general version of the model introduced in Section 1.4. Specifically, we will focus on particle systems of the form

$$
\left\{\begin{aligned}
d X_{t}^{i}= & b\left(t, X_{t}^{i}, \nu_{t}^{N}\right) d t+\sigma\left(t, X_{t}^{i}\right) \sqrt{1-\rho\left(t, \nu_{t}^{N}\right)} d W_{t}^{i} \\
& +\sigma\left(t, X_{t}^{i}\right) \rho\left(t, \nu_{t}^{N}\right) d W_{t}^{0}-\alpha\left(t, X_{t}^{i}, \nu_{t}^{N}\right) d \mathfrak{L}_{t}^{N}, \\
\mathfrak{L}_{t}^{N}= & \left(\mathfrak{K} * L^{N}\right)_{t}, \quad L_{t}^{N}=1-\nu_{t}^{N}(0, \infty), \\
\nu_{t}^{N}= & \sum_{i=1}^{N} a_{i}^{N} \mathbf{1}_{t<\tau_{i}} \delta_{X_{t}^{i}}, \quad \tau_{i}=\inf \left\{t \geq 0: X_{t}^{i} \leq 0\right\},
\end{aligned}\right.
$$

where $\mathfrak{K} \in \mathcal{W}_{0}^{1,1}\left(\mathbb{R}_{+}\right)$and $W^{0}, \ldots, W^{N}$ are independent Brownian motions. Concerning the weights, we assume that there exist $C, c>0$ such that

$$
a_{i}^{N}=\frac{a_{i}\left(X_{0}^{i}\right)}{\sum_{j=1}^{N} a_{j}\left(X_{0}^{j}\right)} \quad \text { with } \quad c \leq a_{i}(\cdot) \leq C \quad \text { for each } \quad i=1, \ldots, N
$$

For the well-posedness of (2.1) we refer to the beginning remarks of Section 3. As regards the model from Section 1.4 , we stress that the drift $b$ depends explicitly on the mean process $M_{t}^{N}:=\left\langle\nu_{t}^{N}, \mathrm{Id}\right\rangle$ and similarly the loss process $L_{t}^{N}$ plays a vital rôle. More generally, these two mean field statistics can serve as useful indicators of systemic risk. However, their convergence as $N \rightarrow \infty$ does not follow directly from our notion of convergence for the empirical measures $\nu^{N}$, so they will require special attention.

\subsection{Assumptions}

In view of Section 1.4 , we need to consider local notions of Lipschitzness in $X_{t}^{i}$ and $\nu_{t}^{N}$, and crucially we must allow the drift to have linear growth in both $X_{t}^{i}$ and $M_{t}^{N}$. To this end, we need suitable notions of distance on the space of sub-probability measures $\mathbf{M}_{\leq 1}(\mathbb{R})$. This leads us to introduce the Kantorovich type distances

$$
\begin{gathered}
d_{0}(\mu, \tilde{\mu}):=\sup \left\{|\langle\mu-\tilde{\mu}, \psi\rangle|:\|\psi\|_{\text {Lip }} \leq 1,|\psi(0)| \leq 1\right\}, \text { and } \\
d_{1}(\mu, \tilde{\mu}):=\sup \left\{|\langle\mu-\tilde{\mu}, \psi\rangle|:\|\psi\|_{\text {Lip }} \leq 1,\|\psi\|_{\infty} \leq 1\right\} .
\end{gathered}
$$

Assumption 2.1 (Structural assumptions). Let the coefficients of (2.1) be of the form $b\left(t, x, \mu, \ell^{\mu}\right), \alpha\left(t, x, \mu, \ell^{\mu}\right), \sigma(t, x)$, and $\rho\left(t, \mu, \ell^{\mu}\right)$ with $\ell^{\mu}:=1-\mu(0, \infty)$. We assume:

(i). (Linear growth and space/time regularity). Let $g=b, \alpha$. The map $x \mapsto g(t, x, \mu, \ell)$ is $\mathcal{C}^{2}(\mathbb{R})$ and $(t, x) \mapsto \sigma(t, x)$ is $\mathcal{C}^{1,2}([0, T] \times \mathbb{R})$. Moreover, there exists $C>0$ s.t.

$$
\begin{aligned}
& |g(t, x, \mu, \ell)| \leq C(1+|x|+\langle\mu,|\cdot|\rangle), \quad\left|\partial_{x}^{(n)} g(t, x, \mu, \ell)\right| \leq C, \quad n=1,2, \\
& |\sigma(t, x)| \leq C, \quad\left|\partial_{t} \sigma(t, x)\right| \leq C, \quad \text { and } \quad\left|\partial_{x}^{(n)} \sigma(t, x)\right| \leq C, \quad n=1,2 .
\end{aligned}
$$

(ii). (Local $d_{0} / d_{1}$-Lipschitzness in $\mu$ ). Let $g=b, \alpha$. There exists $C>0$ s.t.

$$
\begin{aligned}
|g(t, x, \mu, \ell)-g(t, x, \tilde{\mu}, \ell)| & \leq C(1+|x|+\langle\mu,|\cdot|\rangle) d_{0}(\mu, \tilde{\mu}) \\
|\rho(t, \mu, \ell)-\rho(t, \tilde{\mu}, \ell)| & \leq C(1+\langle\mu,|\cdot|\rangle) d_{1}(\mu, \tilde{\mu})
\end{aligned}
$$


(iii). (Piecewise local Lipschitzness in $\ell$ ). There exist $0=\theta_{0}<\cdots<\theta_{k}=1$ s.t.

$$
|g(t, x, \mu, \ell)-g(t, x, \mu, \tilde{\ell})| \leq C(1+|x|+\langle\mu,|\cdot|\rangle)|\ell-\tilde{\ell}|
$$

whenever $\ell, \tilde{\ell} \in\left[\theta_{i-1}, \theta_{i}\right)$ for some $1 \leq i \leq k$, where $g$ is any of $b, \alpha$, or $\rho$.

(iv). (Non-degeneracy). There exists $\epsilon>0$ s.t. $0<\epsilon \leq \sigma(t, x)$ and $0 \leq \rho(t, \mu) \leq 1-\epsilon$.

(v). (Sub-Gaussian initial law). The sequence $X_{0}^{1}, \ldots, X_{0}^{N}$ is i.i.d and independent of the driving Brownian motions. Their common law, $\mu_{0}$, has a density in $L^{2}(0, \infty)$ and

$$
\exists \epsilon>0 \quad \text { s.t. } \quad \mu_{0}(\lambda, \infty)=O\left(\exp \left\{-\epsilon \lambda^{2}\right\}\right) \quad \text { as } \quad \lambda \rightarrow \infty .
$$

Letting $\nu_{0}^{N}:=\sum_{i=1}^{N} a_{i}^{N} \delta_{X_{0}^{i}}$, we assume $\nu_{0}^{N}$ converges weakly to some probability measure $\nu_{0}$ with a density $V_{0} \in L^{2}(0, \infty)$ s.t. $\left\|x V_{0}\right\|_{L^{2}}=\int_{0}^{\infty}\left|x V_{0}(x)\right|^{2} d x<\infty$.

Remark 2.2. In (i) it suffices for $\partial_{x x} b, \partial_{x x} \sigma$ and $\partial_{t} \sigma$ to exist as weak derivatives in $L^{\infty}$, and if $\sigma(t, x)=\sigma_{1}(t) \sigma_{2}(x)$ then no regularity in time is needed. Moreover, the assumption of a common law in (v) is not essential, but this is not the focus here.

For the uniqueness of the limit SPDE we must restrict attention to a class of processes with reasonable regularity and, naturally, we want the limit points of the particle system to be included in this class. As we will see in Section 4 (see also Theorem 2.7), the conditions we impose below are indeed weaker than those that hold for the limit points of $\nu^{N}$.

Assumption 2.3 (Conditions for uniqueness). In Theorem 2.6 we consider the class of càdlàg processes $\tilde{\nu}$ that take values in $\mathbf{M}_{\leq 1}(\mathbb{R})$ and satisfy the following conditions:

(i). (Support on $\left.\mathbb{R}_{+}\right)$. For every $t \in[0, T], \tilde{\nu}_{t}$ is supported on $\mathbb{R}_{+}=[0, \infty)$.

(ii). (Increasing loss). $\tilde{L}_{t}:=1-\tilde{\nu}_{t}(0, \infty)$ is strictly increasing when it is less than 1.

(iii). (Exponential tails). For every $\epsilon>0$, we have

$$
\mathbb{E} \int_{0}^{T} \tilde{\nu}_{t}(a, \infty) d t=o(\exp \{-\epsilon a\}) \quad \text { as } \quad a \rightarrow \infty .
$$

(iv). (Boundary decay). There exists $\beta>0$ such that

$$
\mathbb{E} \int_{0}^{T} \tilde{\nu}_{t}(0, \varepsilon) d t=O\left(\varepsilon^{1+\beta}\right) \quad \text { as } \quad \varepsilon \rightarrow 0 .
$$

(v). (Spatial concentration). There exist $C>0$ and $\delta>0$ such that

$$
\mathbb{E} \int_{0}^{T}\left(\tilde{\nu}_{t}(a, b)\right)^{2} d t \leq C|b-a|^{\delta} \quad \forall a, b \in \mathbb{R} .
$$

\subsection{The limit SPDE}

Let $\mathscr{S}$ denote the space of Schwartz functions on $\mathbb{R}$ and let $\mathscr{S}^{\prime}$ denote its dual, the space of tempered distributions. Then we can think of the empirical measures $\nu^{N}$ as living in the space $D_{\mathscr{S}^{\prime}}=D_{\mathscr{S}^{\prime}}[0, T]$ consisting of $\mathscr{S}^{\prime}$-valued càdlàg processes on $[0, T]$. With a view to exploiting the monotonicity of the loss process, $L^{N}$, it becomes natural to use weak convergence with respect to Skorohod's M1 topology on $D_{\mathscr{S}}$. The extension of the M1 topology to this setting was introduced in [43] and further details can be found there. 
It remains to mention that we consider the limit SPDE in its weak formulation with respect to the space of test functions $\mathscr{C}_{0}$ as defined by

$$
\mathscr{C}_{0}:=\{\phi \in \mathscr{S}: \phi(0)=0\} .
$$

This encodes the idea that the SPDE is posed as a Dirichlet problem on $\mathbb{R}_{+}$.

Theorem 2.4 (Limit SPDE). Suppose Assumption 2.1 is satisfied. Then $\left(\nu^{N}, W^{0}\right)_{N \geq 1}$ is tight on $\left(D_{\mathscr{S}^{\prime}}, \mathrm{M} 1\right)$ and each limit point $\left(\nu, W^{0}\right)$ is a continuous $\mathbf{M}_{\leq 1}\left(\mathbb{R}_{+}\right)$-valued process satisfying Assumption 2.3. Moreover, $\nu$ obeys, with probability 1, the limit SPDE

$$
\begin{aligned}
d\left\langle\nu_{t}, \phi\right\rangle= & \left\langle\nu_{t}, b\left(t, \cdot, \nu_{t}\right) \partial_{x} \phi\right\rangle d t+\frac{1}{2}\left\langle\nu_{t}, \sigma(t, \cdot)^{2} \partial_{x x} \phi\right\rangle d t \\
& +\left\langle\nu_{t}, \sigma(t, \cdot) \rho\left(t, \nu_{t}\right) \partial_{x} \phi\right\rangle d W_{t}^{0}-\left\langle\nu_{t}, \alpha\left(t, \cdot, \nu_{t}\right) \partial_{x} \phi\right\rangle d \mathfrak{L}_{t},
\end{aligned}
$$

for $\phi \in \mathscr{C}_{0}$ and $t \in[0, T]$, where $\mathfrak{L}_{t}:=\int_{0}^{t} \mathfrak{K}(t-s) L_{s} d s$ with $L_{t}:=1-\nu_{t}(0, \infty)$. Finally, if $\nu$ is attained along a subsequence $\left(\nu^{N_{k}}, W^{0}\right)_{k \geq 1}$, then $\left(L^{N_{k}}, W^{0}\right)$ and $\left(M^{N_{k}}, W^{0}\right)$ converge weakly to $\left(L, W^{0}\right)$ and $\left(M, W^{0}\right)$ on $\left(D_{\mathbb{R}}, \mathrm{M} 1\right) \times\left(C_{\mathbb{R}},\|\cdot\|_{\infty}\right)$, where $M_{t}:=\left\langle\nu_{t}, \mathrm{Id}\right\rangle$.

It is instructive to think of the measure-valued SPDE $(2.3)$ in terms of the equation for its density process, $V_{t}$, which is guaranteed to exist in $L^{2}$ by Corollary 5.4 . Based on the boundary decay from Theorem 2.7 below, a simple Borel-Cantelli argument implies $V_{t}(0)=0$ in the sense that $\lim _{\varepsilon \downarrow 0} \varepsilon^{-1} \nu_{t}(0, \varepsilon)=0$, see Lemma A.4. Therefore, integrating by parts in 2.3 leads to the following observation:

Remark 2.5 (SPDE for the density). If $\nu$ is a limit point from Theorem 2.4 then it has a density process $V_{t}$ in $L^{2}\left(\mathbb{R}_{+}\right)$which, in the weak sense, satisfies the Dirichlet problem

$$
\begin{aligned}
d V_{t}(x) & =\frac{1}{2} \partial_{x x}\left(\sigma(t, x)^{2} V_{t}(x)\right) d t-\partial_{x}\left(b\left(t, x, \nu_{t}\right) V_{t}(x)\right) d t \\
& -\rho\left(t, \nu_{t}\right) \partial_{x}\left(\sigma(t, x) V_{t}(x)\right) d W_{t}^{0}+\alpha\left(t, x, \nu_{t}\right) \partial_{x} V_{t}(x) d \mathfrak{L}_{t}, \quad V_{t}(0)=0
\end{aligned}
$$

with $\mathfrak{L}_{t}=(\mathfrak{K} * L)_{t}$ and $L_{t}=1-\int_{0}^{\infty} V_{t}(x) d x$. Assuming sufficient regularity, we can use this SPDE for $V$ and integrate by parts to obtain the formal expression

$$
\frac{d}{d t} \mathfrak{L}_{t}=\frac{1}{2} \int_{0}^{t} \mathfrak{K}(t-s) \sigma(s, 0) \partial_{x} V_{s}(0) d s .
$$

That is, the contagion term driven by $\mathfrak{L}$ acts like an extra transport term proportional to the flux across the boundary - smoothed in time according to $\mathfrak{K}$. In the case of constant coefficients and $\alpha \equiv 0$, sharp regularity results can be found in [42].

Since every limit point of the finite particle system obeys the SPDE (2.3), we can deduce the full weak convergence to this mean-field limit once we have uniqueness of the SPDE in the class of solutions satisfying Assumption 2.3 .

Theorem 2.6 (Uniqueness \& LLN). Let $\left(\nu, W^{0}\right)$ be as in Theorem 2.4 and suppose $\tilde{\nu}$ is another solution to the SPDE (2.3) satisfying Assumption 2.3. Then, with probability 1,

$$
\nu_{t}(A)=\tilde{\nu}_{t}(A) \quad \forall t \in[0, T] \quad \forall A \in \mathcal{B}(\mathbb{R}) .
$$

In particular, a solution to the SPDE 2.3) has a unique law on $\left(D_{\mathscr{S}^{\prime}}, \mathrm{M} 1\right) \times\left(C_{\mathbb{R}},\|\cdot\|_{\infty}\right)$ and $\left(\nu^{N}, W^{0}\right)$ converges weakly to this law. Furthermore, the loss process $L^{N}$ and the mean process $M^{N}$ converge weakly to $L$ and $M$ as defined in Theorem 2.4. 
In view of the pathwise uniqueness, the Yamada-Watanabe theorem ensures that the unique solution to the SPDE can be taken to be strong. Our final result shows that this solution can be recast as the conditional law, given the common Brownian motion $W^{0}$, of a 'conditional' McKean-Vlasov type diffusion with absorbing boundary. Furthermore, we establish an Aronson type upper bound on the density of this absorbed SDE and we show that it has power law decay near the boundary. This is analogous to the classical Dirichlet heat kernel estimates that are available for more standard diffusions (see [34, 12]).

Theorem 2.7 (Conditional McKean-Vlasov formulation). Let $\left(\nu, W^{0}\right)$ be the unique strong solution to the SPDE (2.3). Then, for any Brownian motion $W \perp\left(X_{0}, W^{0}\right)$, we have

$$
\nu_{t}=\mathbb{P}\left(X_{t} \in \cdot, t<\tau \mid W^{0}\right) \quad \text { for } \quad \tau:=\inf \left\{t>0: X_{t} \leq 0\right\},
$$

where $X_{t}$ is the unique solution to the conditional McKean-Vlasov diffusion

$$
\left\{\begin{array}{c}
d X_{t}=b\left(t, X_{t}, \nu_{t}\right) d t+\sigma\left(t, X_{t}\right) \sqrt{1-\rho\left(t, \nu_{t}\right)^{2}} d W_{t} \\
+\sigma\left(t, X_{t}\right) \rho\left(t, \nu_{t}\right) d W_{t}^{0}-\alpha\left(t, X_{t}, \nu_{t}\right) d \mathfrak{L}_{t} \\
\mathfrak{L}_{t}=(\mathfrak{K} * L)_{t}, \quad L_{t}=\mathbb{P}\left(\tau \leq t \mid W^{0}\right), \quad X_{0} \sim \nu_{0}
\end{array}\right.
$$

Moreover, the absorbed process has a density $\mathfrak{p}(t, x)=\mathbb{E} V_{t}(x)$ so that

$$
\mathbb{E} \nu_{t}(a, b)=\mathbb{P}\left(X_{t} \in(a, b), t<\tau\right)=\int_{a}^{b} \mathfrak{p}(t, x) d x,
$$

where, for any $\epsilon>0$, there exists $\kappa \in(0,1)$ and $C, c>0$ such that

$$
\mathfrak{p}(t, x) \leq C \int_{0}^{\infty}\left(\frac{1}{\sqrt{t}}\left(\frac{x}{\sqrt{t}} \wedge 1\right)\left(\frac{y}{\sqrt{t}} \wedge 1\right)+\left(\frac{x^{\kappa} y^{\kappa}}{t^{\kappa}} \wedge 1\right) e^{\epsilon y^{2}}\right)\left(1 \wedge e^{-\frac{(x-y)^{2}}{c t}+c_{x, y}}\right) d \nu_{0}(y)
$$

and

$$
\mathfrak{p}(t, x) \leq C \int_{0}^{\infty}\left(\frac{1}{\sqrt{t}}+e^{\epsilon y^{2}}\right) e^{-\frac{(x-y)^{2}}{c t}} d \nu_{0}(y),
$$

with $c_{x, y} \lesssim|x-y|(x \wedge y)$. Furthermore, if $\sigma(t, x)=\sigma_{1}(t) \sigma_{2}(x)$, then $c_{x, y} \equiv 0$.

Remark 2.8. The bound 2.5) also holds on the whole space. Note that the correction term $c_{x, y}$ in 2.4 is only relevant when $x, y \rightarrow \infty$ jointly (inside a cone determined by $c_{x, y}$ ) and in this case the tail is controlled by 2.5 . Note also that the factor $e^{\epsilon y^{2}}$ requires the initial law to be sub-Gaussian. This is a natural consequence of the linear growth. If e.g. the drift is bounded, then $e^{\epsilon y^{2}}$ can be dropped and $\kappa$ can be taken arbitrarily close to 1 . Similarly, the factor $e^{\epsilon y^{2}}$ drops if the linear growth is in terms of $\left|M_{t}-M_{0}\right|$ and $\left|X_{t}-X_{0}\right|$.

Proof of Theorem 2.7. Given Theorems 2.4 and 2.6. the first claim is straightforward. Indeed, treating $\nu_{t}$ as given, the SDE has a strong solution, $\tilde{X}$, by the standard theory and we can thus define $\tilde{\nu}_{t}:=\mathbb{P}\left(\tilde{X}_{t} \in \cdot, t<\tau \mid W^{0}\right)$. Arguing as in Section 9 of [35], with the obvious changes, we can apply Itô's formula to $\phi\left(\tilde{X}_{t}\right)$, for $\phi \in \mathscr{C}_{0}$, to show that $\tilde{\nu}$ solves a linear version of the SPDE (2.3) with the fixed $\nu$. But then $\tilde{\nu}=\nu$ by uniqueness (for the linear SPDE) which proves the claim. The density estimates (2.4) and (2.5) are more involved, but they will follow from Proposition 4.2 (based on the work in Section 6). 
For the proofs of Theorems 2.4 and 2.6, our techniques build upon and extend the methods of [35], which dealt with a similar SPDE problem, albeit without the contagion term (i.e. $\alpha \equiv 0$ ) and with a bounded drift as well as with coefficients that only depend on $\nu$ via the losses $L$. The main insight from [35] is that it can be fruitful to approach uniqueness via suitable energy estimates in $H^{-1}$ (the dual of the first Sobolev space) when combined with careful control over $\mathbb{E} \nu_{t}(0, \varepsilon)$ as $\varepsilon \downarrow 0$ and $\mathbb{E} \nu_{t}(\lambda, \infty)$ as $\lambda \uparrow \infty$. However, in order for this to work in our setting, several extensions of the arguments in [35] are needed and, crucially, we must rely on novel upper bounds for the densities of the absorbed particles (Proposition 3.3). Ultimately, we thus arrive at the essential $\mathrm{H}^{-1}$ energy estimate (Proposition 5.1 and Lemma 5.2, by establishing power law decay of $x \mapsto \mathbb{E} V_{t}(x)$ near the Dirichlet boundary and Gaussian tails towards infinity (Corollary 3.4).

The proofs of the density bounds are the subject of Section 6 and it is these efforts that lead to the absorbing density estimates in Theorem 2.7. As far as we are aware, these estimates are not available from results elsewhere in the literature, and we believe they are of independent interest. In particular, they have already proved useful in a separate paper [36] related to the problem discussed in Section 2.3.1.

\subsection{Financial contagion and default clustering}

Recall that the nonlinearities of the limit SPDE are of a non-local nature tied to the flux across the origin. This differentiates our setting from the existing theory for Zakai type SPDEs and it has striking consequences for the qualitative behaviour of the solution.

In particular, the health of the financial system - governed by the limit SPDE depends critically on the interplay between the common noise $W^{0}$ and the nonlinear effects of the contagion process $\mathfrak{L}$ (see Fig. 2.1). At least conceptually, this captures the main forces at work in the 2007-2009 financial crisis, where contagion ensued from the correlated corrections across the U.S. housing markets as "financial institutions had levered up on similar large portfolios of securities and loans that faced little idiosyncratic risk, but large amounts of systematic risk" (Acharya et al. [1]).
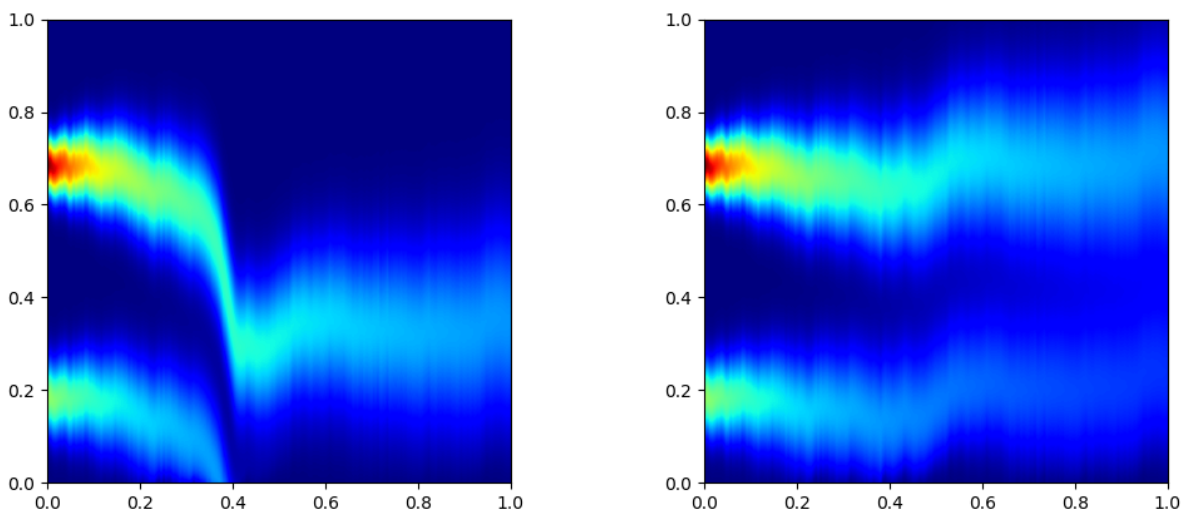

Figure 2.1: The figure shows two heat plots of the density $(t, x) \mapsto V_{t}(x)$ from Remark 2.5, based on a numerical simulation of the SPDE for a fixed realization of $W^{0}$. On the left $\alpha=1.5$, while on the right $\alpha=0$. The other parameters are: $\rho=0.1, \sigma=1, b=0$, and $\mathfrak{K}$ is an isosceles triangle on $[0,0.015]$ with height $2 / 0.015$. The realization of $W^{0}$ starts on a slightly negative trend, but then moves back up again. 
The left-hand plot in Figure 2.1 illustrates how contagion can cause a period of significant default clustering (from $t=0.2$ to $t=0.4$ ), with the right-hand plot confirming that the system would have done just fine in the absence of contagion. Indeed, the entire lower group of 'unhealthy' banks default in the left plot, while the right plot shows a comfortable recovery from the slight initial deterioration (caused by the common exposures).

Notice also that the demise of the lower 'unhealthy' part of the system causes a substantial drop in the distance-to-default of the 'healthier' upper part. However, these problems do not result in further defaults, so the contagious effects die out. As illustrated by Figure 2.2 below, a sharper decline of the common exposures can prompt much more severe contagion, which in turn can result in a default cascade that also wipes out the healthier upper part (over a very short period of time). Finally, we emphasise the critical rôle of the common exposures as an instigator of such periods of high contagion. This is made clear by the rightmost plot of Figure 2.2 , which shows the system trending solidly upwards in a contrasting scenario where the common exposures are doing well.
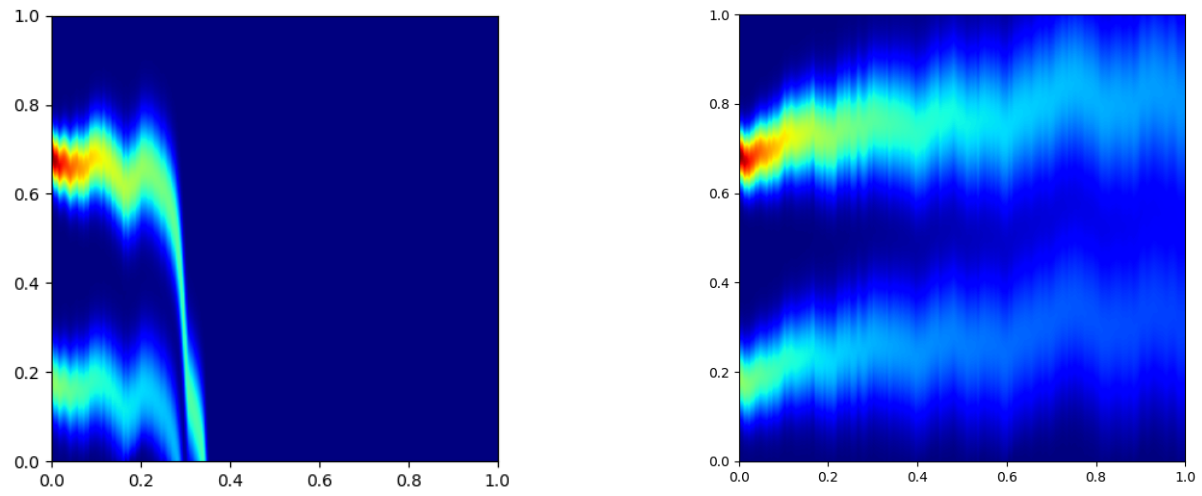

Figure 2.2: In these two heat plots of $(t, x) \mapsto V_{t}(x)$, we have $\alpha=2$ on both sides and otherwise the parameters are the same as in Fig. 2.1 However, the simulations are done for two different realizations of the common noise $W^{0}$ : on the left it declines steadily, while on the right it increases correspondingly.

The importance of the common noise (as portrayed by Fig. 2.2p agrees with observations from the network-based literature, which suggest that idiosyncratic shocks are unlikely to significantly impact a large network, whereas the addition of common shocks can generate substantial losses from contagion (see e.g. the discussion in Cont, Moussa \& Santos [14]). Also, recalling the model from Section 1.4, we note that a high rate of herding can have the dual effect of producing a healthier system in 'normal' times, while serving as an amplifier of potential crises by causing even more default clustering if the common exposures decline significantly. Similarly, the correlation function presents another endogenous channel that can amplify the effect of declining common exposures. We intend to return to a closer study of the interplay between the different parameters in future work.

\subsubsection{Default cascades and the limiting case of instantaneous contagion}

In view of the steep decline in Figure 2.2, it is interesting to consider what happens as the contagious impact of defaults becomes instantaneous. That is, as a sequence of impact kernels $\left(\mathfrak{K}_{\varepsilon}\right)$ approximates the dirac mass at 0 . 
In the case of constant coefficients and no common noise, the resulting limit has recently been studied independently by Nadtochiy \& Shkolnikov [45] and the authors of this paper together with Ledger [36], building on earlier work by Delarue et al. [20, 21]. To be specific, the limiting McKean-Vlasov problem is of the form

$$
X_{t}=X_{0}+b t+\sigma W_{t}-\alpha L_{t}, \quad L_{t}=\mathbb{P}(\tau \leq t),
$$

where $\tau=\inf \left\{t>0: X_{t} \leq 0\right\}$. As it is, 2.6 is ill-posed, however, it is conjectured (see Conj. 1.9 in [36]) that it is well-posed in the class of 'physical' solutions as introduced in [21]. Global existence of a 'physical' solution is known from [21, but uniqueness remains unsettled: If $\alpha$ is sufficiently large (given $X_{0}$ ), $t \mapsto L_{t}$ cannot be continuous [36, Thm. 1.1] and uniqueness is then only known up to the first time the $\mathcal{W}^{1,2}$ norm of $L$ explodes 36 , Thm. 1.8], see also [45. On the other hand, if $\alpha$ is sufficiently small, then it follows from [20. Thm. 2.4] that there is a unique global solution such that $t \mapsto L_{t}$ is in $\mathcal{C}^{1}[0, T]$.

Mathematically, this phase transition in $\alpha$ is very interesting: it means that the steep decline in Figure 2.2 may degenerate into a jump discontinuity whereby a macroscopic part of the system is lost at the blink of an eye. Financially, such a jump could offer an idealized definition of a true 'systemic default cascade', which is the approach adopted in [45] for a variant of (2.6). However, beyond the benefits of a precise definition this may be too categorical, as instantaneous default cascades are not observed in practice and, from a systemic risk point of view, it is the default clustering and steep declines in distances-todefault that matter, not whether they materialized over short periods of time or as jumps. Therefore, we believe the framework proposed in this paper can serve as the reference model, with the instantaneous problem (2.6) arising as an important limiting case.

In addition, there are two theoretical advantages of the model in this paper. Firstly, it needs no extra notion of 'physical' solutions and it makes sense as an SPDE globally. Secondly, the SPDE characterizes the unique limit of the finite system. This latter point is somewhat problematic for (2.6), at least until the aforementioned conjecture is resolved, since global uniqueness is not known and the explosion time (up to which we have uniqueness) may in principle lie strictly before the first jump. In turn, even up to the first jump time, the finite system is not guaranteed to converge to a unique limit, and thus the jump definition of a 'systemic default cascade' cannot be said to rigorously represent the finite financial system. This issue is even more pronounced with non-constant (nonlinear) coefficients and common noise, as nothing is then known about uniqueness.

\subsubsection{Large portfolio credit risk}

Although the focus here is on financial institutions, our framework could also be applied to the study of default clustering in large portfolios of more general defaultable entities. Viewed as a structural large portfolio model for pricing of credit derivatives, our framework extends [35, 9]. In this regard, the idea of having a loss-dependent correlation with finitely many discontinuities (Assumption 2.1, part iii) was considered in 35] as a possible way to accommodate for the implied correlation skew across the tranches of a CDO.

Recently, there has been a great deal of interest in disentangling the rôles of contagion and common risk factors as drivers of corporate default clustering in large portfolios (Azizpour, Giesecke \& Schwenkler [3, Lando \& Nielsen [41, Duffie et al. [23]). However, the existing literature remains inconclusive and has focused almost exclusively on self-exciting point processes, so the model we present here could serve as a first attempt towards a structural platform from which to approach these matters. 


\subsubsection{Connections to mathematical neuroscience}

Interestingly, our setup is closely related to nonlinear leaky integrate-and-fire models for electrically coupled neurons with noisy input. These models can be phrased as particle systems, where each SDE corresponds to the electrical potential of a neuron, and when this potential reaches a threshold voltage, the neuron is then said to spike, thus causing it to fire an electrical signal to the other neurons exciting them to higher voltage levels.

As suggested by Inglis \& Talay [38], the transmission of this signal can be modelled by a cable equation, which translates to a gradual impact of the signal in complete analogy with our contagion mechanism. However, instead of being absorbed at the boundary, the neurons that spike are instantly reset to a predetermined value (called the resting potential) and they then continue to evolve according to this rule ad infinitum. In the mean field limit, this yields a McKean-Vlasov problem analogous to that of Theorem 2.7 except for the resetting of the particle. So far, this has only been studied without common noise (and with simpler interactions) in which case the global well-posedness was proved in [38, building on ideas from [20, 21]. For further background, we refer to [38, 46, 44].

\section{The finite particle system}

We begin by observing that the finite system (2.1) is well-posed for each $N \geq 1$. To see this, we write $\mathbf{X}^{N}=\left(X^{1}, \ldots, X^{N}\right)$ and express the system as a vector-valued SDE

$$
d \mathbf{X}_{t}^{N}=\boldsymbol{b}\left(t, \mathbf{X}_{t}^{N}, \nu_{t}^{N}\right) d t+\boldsymbol{\sigma}\left(t, \mathbf{X}_{t}^{N}\right)\left(\rho_{t} d \mathbf{W}_{t}^{0}+\left(1-\rho_{t}^{2}\right)^{\frac{1}{2}} d \mathbf{W}_{t}\right)-\boldsymbol{\alpha}\left(t, \mathbf{X}_{t}^{N}, \nu_{t}^{N}\right) d \mathfrak{L}_{t}^{N} .
$$

Remark 3.1 (Shorthand notation). Here $\rho_{t}=\rho\left(t, \nu_{t}^{N}\right)$. Similarly, we will sometimes write $\alpha_{t}^{i}=\alpha\left(t, X_{t}^{i}, \nu_{t}^{N}\right)$ and $b_{t}^{i}=b\left(t, X_{t}^{i}, \nu_{t}^{N}\right)$ as well as $\sigma_{t}^{i}=\sigma\left(t, X_{t}^{i}\right)$.

Recall from (iii) of Assumption 2.1 that the Lipschitzness in $L_{t}^{N}$ only holds piecewise. However, on each of the intervals between defaults, $L_{t}^{N}$ is simply equal to a fixed $\mathcal{F}_{0^{-}}$ measurable random variable with $L_{t}^{N}=0$ on $\left[0, \varsigma_{1}\right)$ and then $L_{t}^{N}=\sum_{k=1}^{n} a_{i_{k}}^{N}$ on $\left[\varsigma_{n}, \varsigma_{n+1}\right)$, where $\varsigma_{n}$ is the time of the $n$ 'th default (and $i_{1}, \ldots, i_{n}$ have defaulted). Thus, on each of these intervals we can treat the coefficients as functions of just $t$ and the surviving members of $\mathbf{X}_{t}^{N}$, with (i)-(ii) of Assumption 2.1 giving local Lipschitzness in the euclidean norm. That is, we can solve the system inductively on each of the intervals by running it up to the next default and then restarting it with the new (fixed) value for $L_{t}^{N}$ and with the defaulted particle removed from $\nu_{t}^{N}$. Hence the well-posedness follows by the standard theory for SDEs with locally Lipschitz coefficients of at most linear growth.

\subsection{The finite-dimensional evolution equation}

Since we have sufficient symmetry in the coefficients of the particles, we can obtain a single evolution equation for the dynamics of the empirical measures. Furthermore, the assumptions on the weights $a_{i}^{N}$ ensure that enough averaging is taking place in order for the idiosyncratic noise in this equation to vanish in the large population limit. These observations are made more precise in the next proposition and the work that follows.

Proposition 3.2 (Finite evolution equation). Given $N \geq 1$, it holds for all $\phi \in \mathscr{C}_{0}$ that

$$
\begin{aligned}
d\left\langle\nu_{t}^{N}, \phi\right\rangle=\left\langle\nu_{t}^{N}, b\left(t, \cdot, \nu_{t}^{N}\right) \partial_{x} \phi\right\rangle d t+\frac{1}{2}\left\langle\nu_{t}^{N}, \sigma(t, \cdot)^{2} \partial_{x x} \phi\right\rangle d t & \\
& +\left\langle\nu_{t}^{N}, \sigma(t, \cdot) \rho_{t} \partial_{x} \phi\right\rangle d W_{t}^{0}-\left\langle\nu_{t}^{N}, \alpha_{t} \partial_{x} \phi\right\rangle d \mathfrak{L}_{t}^{N}+d I_{t}^{N}(\phi),
\end{aligned}
$$


where the idiosyncratic driver $I_{t}^{N}$ satisfies

$$
\mathbb{E}\left[\sup _{t \leq T}\left|I_{t}^{N}(\phi)\right|^{2}\right]=O(1 / N) \quad \text { as } \quad N \rightarrow \infty .
$$

Proof. Notice that, since $\phi(0)=0$ for $\phi \in \mathscr{C}_{0}$, we have

$$
\left\langle\nu_{t}^{N}, \phi\right\rangle=\sum_{i=1}^{N} a_{i}^{N} \mathbf{1}_{s<\tau_{i}} \phi\left(X_{t}^{i}\right)=\sum_{i=1}^{N} a_{i}^{N} \phi\left(X_{t \wedge \tau_{i}}^{i}\right), \quad \text { for } \quad \phi \in \mathscr{C}_{0} .
$$

Applying Itô's formula to $\phi\left(X_{t \wedge \tau_{i}}^{i}\right)$, the first result then follows with

$$
I_{t}^{N}(\phi):=\sum_{i=1}^{N} \int_{0}^{t} a_{i}^{N} \sigma\left(s, X_{s}^{i}\right)\left(1-\rho\left(s, \nu_{s}^{N}\right)^{2}\right)^{\frac{1}{2}} \partial_{x} \phi\left(X_{s \wedge \tau_{i}}^{i}\right) d W_{s}^{i} .
$$

Using the independence of the Brownian motions and the boundedness of $\sigma$, we get

$$
\mathbb{E}\left\langle I_{.}^{N}(\phi)\right\rangle_{t}=\sum_{i=1}^{N} \mathbb{E}\left[\int_{0}^{t}\left(a_{i}^{N}\right)^{2}\left(\sigma_{s}^{i}\right)^{2}\left(1-\rho_{s}^{2}\right) \partial_{x} \phi\left(X_{s \wedge \tau_{i}}^{i}\right)^{2} d s\right] \leq C\left\|\partial_{x} \phi\right\|_{\infty}^{2} \sum_{i=1}^{N} \mathbb{E}\left[\left(a_{i}^{N}\right)^{2}\right] .
$$

Thus, the claim follows by Doob's martingale inequality, since $a_{i}^{N} \leq C / N$.

\subsection{Regularity properties of the particles}

In Section 4 we pass to the limit in the finite evolution equation. However, first we need to ensure sufficient regularity at the level of the particles. The cornerstone of this is a family of upper Dirichlet heat kernel type estimates for the densities of the particles.

Proposition 3.3 (Density estimates). Let $X_{t}^{i}$ be given by (2.1) under Assumption 2.1. Then the absorbed process $\left(X_{t}^{i}, t<\tau_{i}\right)$ has a transition density $\mathfrak{p}_{t}^{i, N}$, which satisfies the following bounds: For every $\epsilon>0$ there exist $\kappa \in(0,1)$ and constants $C, c>0$, uniformly in $N \geq 1$ and $t \in(0, T]$, such that

$$
\mathfrak{p}_{t}^{i, N}(x, y) \leq C\left(\frac{1}{\sqrt{t}}\left(\frac{x}{\sqrt{t}} \wedge 1\right)\left(\frac{y}{\sqrt{t}} \wedge 1\right)+\left(\frac{x^{\kappa} y^{\kappa}}{t^{\kappa}} \wedge 1\right) e^{\epsilon y^{2}}\right)\left(1 \wedge e^{-\frac{(x-y)^{2}}{c t}+c_{x, y}}\right)
$$

and

$$
\mathfrak{p}_{t}^{i, N}(x, y) \leq C\left(\frac{1}{\sqrt{t}}+e^{\epsilon y^{2}}\right) e^{-\frac{(x-y)^{2}}{c t}},
$$

where $c_{x, y} \lesssim|x-y|(x \wedge y)$. Furthermore, if $\sigma(t, x)=\sigma_{1}(t) \sigma_{2}(x)$, then $c_{x, y} \equiv 0$.

Proof. The proof is postponed to Section 6. For the final proof, see Section 6.4.

The above estimates provide critical control over the first moment of the mass of $\nu^{N}$ near the boundary as well as Gaussian decay towards infinity (see Corollary 3.4 below). As we verify in Proposition 4.3, these features carry over to the limit points of the particle system and this will be essential to the proof of the energy estimates in Section 5.

Corollary 3.4 (Regularity of the empirical measures). The empirical measures $\nu^{N}$ satisfy $\mathbb{E} \nu_{t}^{N}(a, b) \leq C t^{-\frac{1}{2}}|b-a|$, and it holds uniformly in $N \geq 1$ and $t \in(0, T]$ that

$$
\left\{\begin{array}{lll}
\exists \epsilon>0: & \mathbb{E} \nu_{t}^{N}(a, \infty)=O\left(\exp \left\{-\epsilon a^{2}\right\}\right) & \text { as } \quad a \rightarrow \infty \\
\exists \delta \in(0,1], \beta>0: & \mathbb{E} \nu_{t}^{N}(0, \varepsilon)=t^{-\frac{\delta}{2}} O\left(\varepsilon^{1+\beta}\right) \quad \text { as } & \varepsilon \rightarrow 0
\end{array}\right.
$$


Proof. Recalling the definition of $\nu_{t}^{N}$ and using that $a_{i}^{N} \leq C / N$, it follows from Proposition 3.3 that, for any $(a, b) \subseteq \mathbb{R}_{+}$, we have

$$
\mathbb{E} \nu_{t}^{N}(a, b) \leq \frac{C}{N} \sum_{i=1}^{N} \mathbb{P}\left(X_{t \wedge \tau_{i}}^{i} \in(a, b)\right) \leq C^{\prime} \int_{0}^{\infty} \int_{a}^{b} f_{t}(x, y) d x d \mu_{0}(y),
$$

where $f_{t}(x, y)$ can take the value of either of the right-hand sides of $(3.2)$ or $(3.3)$. Given (3.3) and the sub-Gaussianity of $\mu_{0}$, the first two claims are immediate. Similarly, the final claim follows from $(3.2)$ by exploiting the power law decay at the boundary for $t>0$.

\subsection{Regularity properties of the loss process}

Below we present two important results about the limiting behaviour of the loss process. The first result assures that, in the large population limit, it is strictly increasing when there is mass left in the system. This is crucial for the convergence to the limit SPDE (Section 4.2 and for uniqueness (Section 5.1.1) as it ensures the loss process cannot get stuck at one of the coefficients' finitely discontinuity points (see Assumption 2.1(iii)).

Proposition 3.5. For any $t \in[0, T)$ and $h>0$, it holds for all $r<1$ that

$$
\lim _{\delta \rightarrow 0} \limsup _{N \rightarrow \infty} \mathbb{P}\left(L_{t+h}^{N}-L_{t}^{N}<\delta, L_{t}^{N}<r\right)=0 .
$$

Proof. See Section A.2 in the Appendix.

While the system is progressively losing mass by the previous proposition, the next result ensures that there cannot be too large losses in arbitrarily small amounts of time. This is used in the tightness arguments below (Section 4).

Proposition 3.6. For every $t \in[0, T]$ and $\eta>0$, we have

$$
\lim _{\delta \rightarrow 0} \lim _{N \rightarrow \infty} \mathbb{P}\left(L_{t+\delta}^{N}-L_{t}^{N} \geq \eta\right)=0 .
$$

Proof. See Section A.2 in the Appendix.

The above relies on the impact kernel $\mathfrak{K}$ being in $\mathcal{W}^{1,1}$. As discussed in Section 2.3.1. instantaneous contagion can imply jumps in the loss process with positive probability.

\section{Tightness and convergence}

The aim of this section is to recover the SPDE (2.3) by passing to the limit in the finite evolution equation (3.1). To achieve this, we need to establish tightness of $\left(\nu^{N}\right)$ and then we need some continuity results for the integrals in (3.1). The first step towards tightness is to control the increments of the particles.

Lemma 4.1. For all $s, t \in[0, T]$, it holds uniformly in $N \geq 1$ and $i=1, \ldots, N$ that

$$
\mathbb{E}\left[\left|X_{t \wedge \tau_{i}}^{i}-X_{s \wedge \tau_{i}}^{i}\right|^{4}\right]=O\left(|t-s|^{2}\right) \quad \text { as } \quad|t-s| \rightarrow 0,
$$

where $\tau_{i}=\inf \left\{t>0: X_{t}^{i} \leq 0\right\}$. 
Proof. Using the equation for $X_{t}^{i}$ with $d B_{t}^{i}=\sqrt{1-\rho_{t}^{2}} d W_{t}^{i}+\rho_{t} d W_{t}^{0}$, we have

$$
\mathbb{E}\left[\left|X_{t \wedge \tau_{i}}^{i}-X_{s \wedge \tau_{i}}^{i}\right|^{4}\right] \lesssim \mathbb{E}\left[\left(\int_{s}^{t} \sigma_{r} \mathbf{1}_{r<\tau_{i}} d B_{r}^{i}\right)^{4}\right]+\mathbb{E}\left[\left(\int_{s}^{t}\left|b_{r}^{i}-\alpha_{r}^{i}\left(\mathfrak{L}^{N}\right)_{t}^{\prime}\right| d r\right)^{4}\right]
$$

Since $\sigma_{u}$ is bounded, the Burkholder-Davis-Gundy inequality yields

$$
\mathbb{E}\left[\left(\int_{s}^{t} \sigma_{r} \mathbf{1}_{r<\tau_{i}} d B_{r}^{i}\right)^{4}\right] \lesssim \mathbb{E}\left[\left\langle B .-B_{s}\right\rangle_{t}^{2}\right]=(t-s)^{2} .
$$

Letting $\Lambda_{r}^{i, N}:=\left|X_{t}^{i}\right|+\sum_{j=1}^{N} a_{j}^{N}\left|X_{t}^{j}\right|$, the conditions in Assumption 2.1 imply the bound $\left|b_{r}^{i}\right|+\left|\alpha_{r}^{i}\right| \lesssim 1+\Lambda_{r}^{i, N}$. Noting also that $\left(\mathfrak{L}^{N}\right)^{\prime} \in L^{\infty}$, Jensen's inequality gives

$$
\mathbb{E}\left[\left(\int_{s}^{t}\left|b_{r}^{i}-\alpha_{r}^{i}\left(\mathfrak{L}^{N}\right)_{r}^{\prime}\right| d r\right)\right] \lesssim \mathbb{E}\left[\left(\int_{s}^{t}\left(1+\Lambda_{r}^{i, N}\right) d r\right)^{4}\right] \leq(t-s)^{4} \sup _{r \leq T} \mathbb{E}\left[\left(1+\Lambda_{r}^{i, N}\right)^{4}\right],
$$

where last term is bounded uniformly, by 3.3 of Proposition 3.3 .

Proposition 4.2 (Tightness). The sequence $\left(\nu^{N}, W^{0}\right)$ is tight on $\left(D_{\mathscr{S}^{\prime}}, \mathrm{M} 1\right) \times\left(C_{\mathbb{R}},\|\cdot\|_{\infty}\right)$ and any limit point $\nu^{*}$ is $\mathbf{M}_{\leq 1}\left(\mathbb{R}_{+}\right)$-valued. Moreover, if we set $L_{t}^{*}:=1-\nu_{t}^{*}(0, \infty)$, then $L^{*}$ is strictly increasing when $L^{*}<1$, and $\left(L^{N}, W^{0}\right)$ converges weakly to $\left(L^{*}, W^{0}\right)$ on $\left(D_{\mathbb{R}}, \mathrm{M} 1\right) \times\left(C_{\mathbb{R}},\|\cdot\|_{\infty}\right)$ whenever $\left(\nu^{N}, W^{0}\right)$ converges weakly to $\left(\nu^{*}, W^{0}\right)$.

Proof. For the tightness of $\left(\nu^{N}, W^{0}\right)$, it suffices to show the M1 tightness of $\left\langle\nu^{N}, \phi\right\rangle$ on $D_{\mathbb{R}}$ for every $\phi \in \mathscr{S}$, by Theorem 3.2 in [43]. To this end, we verify the sufficient conditions (i) and (ii) from Theorem 12.12.3 in [51]. The first condition is trivial since $\left\langle\nu^{N}, \phi\right\rangle$ is uniformly bounded by $\left|\left\langle\nu^{N}, \phi\right\rangle\right| \leq\|\phi\|_{\infty}$ for all $N \geq 1$.

For the second condition, we can consider the decomposition

$$
\left\langle\nu_{t}^{N}, \phi\right\rangle=\left\langle\hat{\nu}_{t}^{N}, \phi\right\rangle-\phi(0) L_{t}^{N} \quad \text { with } \quad \hat{\nu}_{t}^{N}:=\sum_{i=1}^{N} a_{i}^{N} \delta_{X_{t \wedge \tau_{i}}^{i}} .
$$

The advantage of this is that the monotone part, $\phi(0) L_{t}^{N}$, is immaterial to the M1 modulus of continuity. Indeed, by Propositions 4.1 and 4.2 of [43], it is sufficient to verify that

$$
\mathbb{E}\left[\left|\left\langle\hat{\nu}_{t}^{N}, \phi\right\rangle-\left\langle\hat{\nu}_{s}^{N}, \phi\right\rangle\right|^{4}\right]=O\left(|t-s|^{2}\right) \quad \forall s, t \in[0, T]
$$

and that, for every $\varepsilon>0$,

$$
\lim _{\delta \rightarrow 0} \lim _{N \rightarrow \infty} \mathbb{P}\left(\sup _{t \in(0, \delta)}\left|\left\langle\nu_{t}^{N}-\nu_{0}^{N}, \phi\right\rangle\right|+\sup _{t \in(T-\delta, T)}\left|\left\langle\nu_{T}^{N}-\nu_{t}^{N}, \phi\right\rangle\right|>\varepsilon\right)=0 .
$$

Recalling $a_{i}^{N} \leq C / N$, Jensen's inequality and the Lipschitzness of $\phi \in \mathscr{S}$ imply

$$
\mathbb{E}\left[\left|\left\langle\hat{\nu}_{t}^{N}, \phi\right\rangle-\left\langle\hat{\nu}_{s}^{N}, \phi\right\rangle\right|^{4}\right] \leq \frac{C}{N}\|\phi\|_{\text {Lip }} \sum_{i=1}^{N} \mathbb{E}\left[\left|X_{t \wedge \tau_{i}}^{i}-X_{s \wedge \tau_{i}}^{i}\right|^{4}\right] .
$$

Thus, we can conclude from Lemma 4.1 that 4.2 is satisfied. With regard to 4.3 , the decomposition 4.1. yields

$$
\mathbb{P}\left(\sup _{t \in(0, \delta)}\left|\left\langle\nu_{t}^{N}-\nu_{0}^{N}, \phi\right\rangle\right|>\varepsilon\right) \leq \mathbb{P}\left(\sup _{t \in(0, \delta)}\left|\left\langle\hat{\nu}_{t}^{N}-\hat{\nu}_{0}^{N}, \phi\right\rangle\right| \geq \frac{\varepsilon}{2}\right)+\mathbb{P}\left(|\phi(0)| L_{\delta}^{N} \geq \frac{\varepsilon}{2}\right)
$$


and likewise for the supremum over $t \in(T-\delta, T)$. By Markov's inequality and the same arguments as above, the first term vanishes uniformly in $N \geq 1$ as $\delta \rightarrow 0$. Combining this with Proposition 3.6, we deduce (4.3). Therefore, $\left(\nu^{N}, W^{0}\right)$ is tight, as desired.

For the final claims, Proposition 5.3 of [35] ensures that each limit point $\nu_{t}^{*}$ can be recovered as an element of $\mathbf{M}_{\leq 1}\left(\mathbb{R}_{+}\right)$. Moreover, using Propositions 3.5 and 3.6 , the claims about $L^{*}$ and $L^{N}$ follow by arguing as in Propositions 5.5 and 5.6 of [35].

Proposition 4.3 (Limit point regularity). For every $\epsilon>0$ there exist $\kappa \in(0,1)$ and $c>0$ such that any continuous limit point $\nu^{*}$ of $\left(\nu^{N}\right)$ satisfies

$$
\left\{\begin{array}{l}
\mathbb{E} \nu_{t}^{*}(a, b) \lesssim \int_{0}^{\infty} \int_{a}^{b}\left(\frac{1}{\sqrt{t}}\left(\frac{x}{\sqrt{t}} \wedge 1\right)\left(\frac{y}{\sqrt{t}} \wedge 1\right)+\left(\frac{x^{\kappa} y^{\kappa}}{t^{\kappa}} \wedge 1\right) e^{\epsilon y^{2}}\right)\left(1 \wedge e^{-\frac{(x-y)^{2}}{c t}+c_{x, y}}\right) d x d \nu_{0}(y) \\
\mathbb{E} \nu_{t}^{*}(a, b) \lesssim \int_{0}^{\infty} \int_{a}^{b}\left(\frac{1}{\sqrt{t}}+e^{\epsilon y^{2}}\right) e^{-\frac{(x-y)^{2}}{c t}} d x d \nu_{0}(y),
\end{array}\right.
$$

for any $(a, b) \subseteq \mathbb{R}_{+}$. Here $c_{x, y} \lesssim|x-y|(x \wedge y)$, and if $\sigma(t, x)=\sigma_{1}(t) \sigma_{2}(x)$, then $c_{x, y} \equiv 0$. In particular, $\nu^{*}$ satisfies Assumption 2.3 with the pointwise properties

$$
\left\{\begin{array}{lll}
\exists \epsilon>0: & \mathbb{E} \nu_{t}^{*}(\lambda, \infty)=O\left(\exp \left\{-\epsilon \lambda^{2}\right\}\right) & \text { as } \quad \lambda \rightarrow \infty \\
\exists \delta \in(0,1], \beta>0: & \mathbb{E} \nu_{t}^{*}(0, \varepsilon)=t^{-\frac{\delta}{2}} O\left(\varepsilon^{1+\beta}\right) \quad \text { as } & \varepsilon \rightarrow 0
\end{array}\right.
$$

Proof. Fix an interval $(a, b) \subseteq \mathbb{R}_{+}$and fix a small $\varepsilon>0$ s.t. $a-\varepsilon>0$. Let $\varphi_{\varepsilon}$ be a smooth cut-off function in $\mathcal{C}_{c}^{\infty}(\mathbb{R} ;[0,1])$ equal to 1 on $(a, b)$ and zero outside $(a-\varepsilon, b+\varepsilon)$. Then, as in the proof of Corollary 3.4, we have

$$
\mathbb{E}\left\langle\nu_{t}^{N}, \varphi_{\varepsilon}\right\rangle \leq \mathbb{E} \nu_{t}^{N}(a-\varepsilon, b+\varepsilon) \leq C \int_{0}^{\infty} \int_{a-\varepsilon}^{b+\varepsilon} f_{t}(x, y) d x d \nu_{0}(y),
$$

where $f_{t}(x, y)$ can take the value of either of the right-hand sides of $(3.2)$ and 3.3 . Here we have also used that $\mu_{0}$ is dominated by a constant times $\nu_{0}=\lim _{N} \sum_{i=1}^{N} a_{i}^{N} \delta_{X_{0}^{i}}$, in light of 2.2 . Since $\varphi_{\varepsilon} \in \mathscr{S}$, after passing to a convergent subsequence, $\left\langle\nu^{N_{k}}, \varphi_{\varepsilon}\right\rangle$ converges weakly to $\left\langle\nu^{*}, \varphi_{\varepsilon}\right\rangle$ on $D_{\mathbb{R}}$ by [43, Prop. 2.7]. Thus, by the assumption that $\nu^{*}$ is continuous, [51, Thm. 12.4.1] gives that $\left\langle\nu_{t}^{N_{k}}, \varphi_{\varepsilon}\right\rangle$ converges weakly to $\left\langle\nu_{t}^{*}, \varphi_{\varepsilon}\right\rangle$ on $\mathbb{R}$ for all $t \in[0, T]$. Using bounded convergence, we get convergence of the means and hence, for all $\varepsilon>0$,

$$
\mathbb{E} \nu_{t}^{*}(a, b) \leq \mathbb{E}\left\langle\nu_{t}^{*}, \varphi_{\varepsilon}\right\rangle=\lim _{k \rightarrow \infty} \mathbb{E}\left\langle\nu_{t}^{N_{k}}, \varphi_{\varepsilon}\right\rangle
$$

Recalling (4.4) and applying dominated convergence, the proof is complete.

Remark 4.4. If $\nu^{*}$ is not continuous, it still holds that $\int_{0}^{T}\left\langle\nu_{t}^{N_{k}}, \varphi_{\varepsilon}\right\rangle d t \Rightarrow \int_{0}^{T}\left\langle\nu_{t}^{*}, \varphi_{\varepsilon}\right\rangle d t$, by [51, Thm. 11.5.1], where ' $\Rightarrow$ ' denotes weak convergence. Hence Assumption 2.3 holds for every limit point of $\left(\nu^{N}\right)$ without any a priori knowledge of continuity.

\subsection{Convergence of the mean process}

Let $M_{t}^{N}:=\left\langle\nu_{t}^{N}, \psi\right\rangle$ for any given Lipschitz function $\psi \in \operatorname{Lip}(\mathbb{R})$. While we are mainly interested in the case $\psi=\mathrm{Id}$, other applications may call for a general Lipschitz function, so we allow for this in the ensuing arguments.

Given $\nu^{N} \Rightarrow \nu^{*}$ on $\left(D_{\mathscr{S}^{\prime}}, \mathrm{M} 1\right)$, where ' $\Rightarrow$ ' denotes weak convergence, we would like to know that $M^{N} \Rightarrow M^{*}:=\left\langle\nu^{*}, \psi\right\rangle$ on $\left(D_{\mathbb{R}}, \mathrm{M} 1\right)$. However, $\psi$ need not be in $\mathscr{S}$, so we 
cannot simply appeal to the continuity of the projection map from $\left(D_{\mathscr{S}^{\prime}}, \mathrm{M} 1\right)$ to $\left(D_{\mathbb{R}}, \mathrm{M} 1\right)$. Nevertheless, we can work around this obstacle by exploiting the uniformly exponential tails of the empirical measures in expectation, cf. Proposition 3.3 .

First of all, we can observe that $\left(M^{N}\right)_{N \geq 1}$ is tight on $\left(D_{\mathbb{R}}, \mathrm{M} 1\right)$. To see this, we verify the necessary conditions (i) and (ii) from Theorem 12.12.3 in [51]. The first condition amounts to showing that, for all $\varepsilon>0$, there exists $c>0$ such that

$$
\mathbb{P}\left(\sup _{t \leq T}\left|M_{t}^{N}\right|>c\right) \leq \varepsilon \quad \forall N \geq 1 .
$$

This property is immediate, since we even have uniformly sub-Gaussian tails of the running max of $M_{t}^{N}$, by Corrollary 6.2 For the second condition, we can rely on the decomposition (4.1), which amounts to

$$
M_{t}^{N}=\hat{M}_{t}^{N}-\psi(0) L_{t}^{N}, \quad \hat{M}_{t}^{N}:=\left\langle\hat{\nu}_{t}^{N}, \psi\right\rangle .
$$

Since $\psi$ is Lipschitz, the tightness then follows by repeating the same arguments as in the proof of Proposition 4.2. Given this, we can now address the weak convergence.

Proposition 4.5 (Functional LLN for the mean). Suppose $\left(\nu^{N}, W^{0}\right) \Rightarrow\left(\nu^{*}, W^{0}\right)$. Then $\left(M^{N}, W^{0}\right) \Rightarrow\left(M^{*}, W^{0}\right)$ on $\left(D_{\mathbb{R}}, \mathrm{M} 1\right) \times\left(C_{\mathbb{R}},\|\cdot\|_{\infty}\right)$.

Proof. Let $\left(M^{N_{k}}, W^{0}\right)_{k \geq 1}$ be an arbitrary subsequence. By the tightness of $\left(M^{N_{k}}\right)$, we can pass to a further convergent subsequence, also indexed by $k \geq 1$. Let $\left(M^{\dagger}, W^{0}\right)$ denote the weak limit of this subsequence and note that we still have $\nu^{N_{k}} \Rightarrow \nu^{*}$. We need to show that the laws of $\left(M^{*}, W^{0}\right)$ and $\left(M^{\dagger}, W^{0}\right)$ agree. To this end, let $\phi_{\lambda}:=\varphi_{\lambda} \psi$, where $\varphi_{\lambda} \in \mathcal{C}_{c}^{\infty}(\mathbb{R} ;[0,1])$ is a standard cutoff function equal to 1 on $[-\lambda, \lambda]$. Then $\phi_{\lambda} \in \mathscr{S}(\mathbb{R})$ and $\phi_{\lambda} \rightarrow \psi$ pointwise as $\lambda \rightarrow \infty$. Since $\phi_{\lambda} \in \mathscr{S}$, the projection map $\pi^{\phi_{\lambda}}:\left(D_{\mathscr{S}^{\prime}}, \mathrm{M} 1\right) \rightarrow$ $\left(D_{\mathbb{R}}, \mathrm{M} 1\right)$ is continuous (see [43, Prop. 2.7]), so we get

$$
\left\langle\nu^{N_{k}}, \phi_{\lambda}\right\rangle \Rightarrow\left\langle\nu^{*}, \phi_{\lambda}\right\rangle \quad \text { on } \quad\left(D_{\mathbb{R}}, \mathrm{M} 1\right),
$$

by the continuous mapping theorem. Moreover, for all $\lambda>0$, we have

$$
\mathbb{E}\left|\left\langle\nu_{t}^{N_{k}}, \psi-\phi_{\lambda}\right\rangle\right| \leq \mathbb{E}\left\langle\nu_{t}^{N_{k}},|\psi| \mathbf{1}_{[\lambda, \infty)}\right\rangle \lesssim \mathbb{E}\left\langle\nu_{t}^{N_{k}},(1+|x|) \mathbf{1}_{[\lambda, \infty)}\right\rangle,
$$

and hence the exponential tails (in expectation) imply

$$
\mathbb{E}\left|\left\langle\nu_{t}^{N_{k}}, \psi-\phi_{\lambda}\right\rangle\right|=o(1) \quad \text { as } \lambda \rightarrow \infty, \quad \text { uniformly in } k \geq 1, t \in[0, T],
$$

see Lemma A.3. Next, we define

$$
\mathbb{T}:=\operatorname{cont}\left(M^{\dagger}\right) \cap \bigcap_{\lambda \in \mathbb{N}} \operatorname{cont}\left(\left\langle\nu^{*}, \phi_{\lambda}\right\rangle\right), \quad \operatorname{cont}(\xi)=\left\{t \in[0, T]: \mathbb{P}\left(\xi_{t-}=\xi_{t}\right)=1\right\},
$$

and note that this set is co-countable since $\left\langle\nu^{*}, \phi_{\lambda}\right\rangle$ and $M^{\dagger}$ are in $D_{\mathbb{R}}$ (see e.g. [51, Cor. 12.2.1]). Given this, we define $\widetilde{\mathbb{T}}:=\mathbb{T} \cap\left\{t \in[0, T]: \mathbb{P}\left(M_{t}^{*}<\infty\right)=1\right\}$, where the latter set has full Lebesgue measure, so $\widetilde{\mathbb{T}}$ is dense in $[0, T]$. We will show that all the finite dimensional distributions of $\left(M^{*}, W^{0}\right)$ and $\left(M^{\dagger}, W^{0}\right)$ agree for indices $\left\{t_{1}, \ldots, t_{l}\right\} \in \widetilde{\mathbb{T}}$. By a monotone class argument, it suffices to show that

$$
\mathbb{E}\left[\prod_{i=1}^{l} f_{i}\left(M_{t_{i}}^{*}\right) g_{i}\left(W_{t_{i}}^{0}\right)\right]=\lim _{k \rightarrow \infty} \mathbb{E}\left[\prod_{i=1}^{l} f_{i}\left(M_{t_{i}}^{N_{k}}\right) g_{i}\left(W_{t_{i}}^{0}\right)\right]
$$


for bounded functions $f_{i}, g_{i} \in \operatorname{Lip}\left(\mathbb{R} ; \mathbb{R}_{+}\right), i=1, \ldots, l$, where we have used convergence of the marginals $\left(M_{t_{i}}^{N_{k}}, W_{t_{i}}^{0}\right)_{i=1, \ldots, l} \Rightarrow\left(M_{t_{i}}^{\dagger}, W_{t_{i}}^{0}\right)_{i=1, \ldots, l}$, see [51, Thm. 11.6.6].

Recalling $M_{t_{i}}^{N_{k}}=\left\langle\nu_{t_{i}}^{N_{k}}, \psi\right\rangle$, we can observe that

$$
\left|f_{i}\left(M_{t_{i}}^{N_{k}}\right)-f_{i}\left(\left\langle\nu_{t_{i}}^{N_{k}}, \phi_{\lambda}\right\rangle\right)\right| \leq\left\|f_{i}\right\|_{\text {Lip }}\left|\left\langle\nu_{t_{i}}^{N_{k}}, \psi-\phi_{\lambda}\right\rangle\right|, \quad i=1, \ldots, l .
$$

Using the upper bounds on each $f_{i}\left(M_{t_{i}}^{N_{k}}\right)$, as implied by (4.8), it follows from (4.6) and the boundedness of $f_{i}, g_{i} \geq 0$, that

$$
\mathbb{E}\left[\prod_{i=1}^{l} f_{i}\left(M_{t_{i}}^{N_{k}}\right) g_{i}\left(W_{t_{i}}^{0}\right)\right] \leq \mathbb{E}\left[\prod_{i=1}^{l} f_{i}\left(\left\langle\nu_{t_{i}}^{N_{k}}, \phi_{\lambda}\right\rangle\right) g_{i}\left(W_{t_{i}}^{0}\right)\right]+\{o(1) \text { terms }\},
$$

as $\lambda \rightarrow \infty$, where the $o(1)$ terms are uniform in $k \geq 1$. By construction of $\widetilde{\mathbb{T}},[51$, Thm. 12.4.1] implies that the canonical projections $\pi_{t_{i}}:\left(D_{\mathbb{R}}, \mathrm{M} 1\right) \rightarrow \mathbb{R}$ are continuous at each $\left\langle\nu^{N_{k}}, \phi_{\lambda}\right\rangle$ for $\lambda \in \mathbb{N}$. Thus, with $\left\langle\nu_{t_{i}}^{N_{k}}, \phi_{\lambda}\right\rangle=\pi_{t_{i}}\left\langle\nu^{N_{k}}, \phi_{\lambda}\right\rangle$, the weak convergence 4.5 implies that, by taking a limsup over $k \geq 1$ on both sides of 4.9 ,

$$
\lim _{k \rightarrow \infty} \mathbb{E}\left[\prod_{i=1}^{l} f_{i}\left(M_{t_{i}}^{N_{k}}\right) g_{i}\left(W_{t_{i}}^{0}\right)\right] \leq \mathbb{E}\left[\prod_{i=1}^{l} f_{i}\left(\left\langle\nu_{t_{i}}^{*}, \phi_{\lambda}\right\rangle\right) g_{i}\left(W_{t}^{0}\right)\right]+o(1) \quad \text { as } \lambda \rightarrow \infty .
$$

Finally, we note that $\left\langle\nu_{t}^{*}, \phi_{\lambda}\right\rangle \rightarrow\left\langle\nu_{t}^{*}, \psi\right\rangle=M_{t}^{*}$ as $\lambda \rightarrow \infty$, by dominated convergence, since $\left|\phi_{\lambda}\right| \leq|\psi|$ and $\psi$ is integrable with respect to $\nu_{t}^{*}$ for $t \in \widetilde{\mathbb{T}}$. Consequently, by the boundedness and continuity of each $\left(f_{i}, g_{i}\right)$, we can send $\lambda \rightarrow \infty$ to find that

$$
\lim _{k \rightarrow \infty} \mathbb{E}\left[\prod_{i=1}^{l} f_{i}\left(M_{t_{i}}^{N_{k}}\right) g_{i}\left(W_{t_{i}}^{0}\right)\right] \leq \mathbb{E}\left[\prod_{i=1}^{l} f_{i}\left(M_{t_{i}}^{*}\right) g_{i}\left(W_{t_{i}}^{0}\right)\right] .
$$

Now, if we had instead relied on the lower bounds for each $f_{i}\left(M_{t_{i}}^{N_{k}}\right)$ implied by 4.8 , then the analogous arguments would yield the reverse inequality, thus proving (4.7).

\subsection{The limit SPDE - Proof of Theorem 2.4}

By [43, Thm. 3,2], the tightness from Proposition 4.2 implies relative compactness in the sense that every subsequence of $\left(\nu^{N}, W^{0}\right)$ has a weakly convergent subsequence. Using this, we show that the corresponding integrals in the finite evolution equation (3.1) converge weakly (Proposition 4.7) and then we employ a martingale argument (Proposition 4.8) to see that this gives rise to the desired limit SPDE.

Remark 4.6 (Skorokhod's representation theorem). While $\left(D_{\mathscr{S}^{\prime}}, \mathrm{M} 1\right)$ fails to be Polish, it is a completely regular Suslin space. In particular, condition (10) of [39, Thm. 2] holds and hence any weakly convergent subsequence of $\left(\nu^{N}\right)$ has a further subsequence with the usual a.s. Skorokhod representation property. Firstly, $\left(D_{\mathscr{S}^{\prime}}, \mathrm{M} 1\right)$ is completely regular and Hausdorff by [43, Prop. 2.7] and [40, Thm. 2.1.1]. Secondly, [43, Prop. 2.7] and its proof gives $D_{\mathscr{S}^{\prime}}=\bigcup_{n=1}^{\infty} D_{S_{-n}}$ where each $\left(D_{S_{-n}}, \mathrm{M} 1\right)$ is Polish and the inclusions $D_{S_{-n}} \hookrightarrow D_{\mathscr{S}^{\prime}}$ are M1-continuous. Thus, each $D_{S_{-n}}$ is Suslin in the Hausdorff space $\left(D_{\mathscr{S}^{\prime}}, \mathrm{M} 1\right)$ and so their union is Suslin under the finest topology such that the inclusions are continuous [48, Thm. I.II.3]. As the M1-topology is coarser, we conclude that $\left(D_{\mathscr{S}^{\prime}}, \mathrm{M} 1\right)$ is Suslin. 
Proposition 4.7 (Convergence of integrals). Fix $t \leq T$ and $\phi \in \mathscr{S}$, and define

$$
\begin{gathered}
\Psi, \Phi:\left\{\zeta \in D_{\mathscr{S}^{\prime}}: \zeta_{s} \in \mathbf{M}_{\leq 1}(\mathbb{R})\right\} \times D_{\mathbb{R}} \rightarrow \mathbb{R} \quad \text { by } \\
\Psi(\zeta, \ell):=\int_{0}^{t}\left\langle\zeta_{s}, g\left(s, \cdot, \zeta_{s}, \ell_{s}\right) \phi\right\rangle d s, \Phi(\zeta, \ell):=\int_{0}^{t}\left\langle\zeta_{s}, \alpha\left(s, \cdot, \zeta_{s}, \ell_{s}\right) \phi\right\rangle d(\mathfrak{K} * \ell)_{s},
\end{gathered}
$$

where $g$ is any of $b, \sigma^{2}$, or $\sigma \rho$. Given $\left(\nu^{N}, L^{N}\right) \Rightarrow\left(\nu^{*}, L^{*}\right)$ on $\left(D_{\mathscr{S}^{\prime}}, \mathrm{M} 1\right) \times\left(D_{\mathbb{R}}, \mathrm{M} 1\right)$, $\Psi^{N}:=\Psi\left(\nu^{N}, L^{N}\right)$ converges weakly to $\Psi^{*}:=\Psi\left(\nu^{*}, L^{*}\right)$, on $\mathbb{R}$, and likewise for $\Phi$.

Proof. Fix a bounded $f \in \operatorname{Lip}(\mathbb{R})$. By Remark 4.6 any subsequence of $\left(\nu^{N}, L^{N}\right)$ has a further subsequence, also indexed by $N$, for which we can assume almost sure convergence. By applying the triangle inequality, we have

$$
\begin{aligned}
\mid \mathbb{E} f\left(\Psi^{N}\right)- & \mathbb{E} f\left(\Psi^{*}\right)|\lesssim \mathbb{E}| \int_{0}^{t}\left\langle\nu_{s}^{*}-\nu_{s}^{N}, g\left(s, \cdot, \nu_{s}^{*}, L_{s}^{*}\right) \phi\right\rangle d s \mid \\
& +\mathbb{E}\left|\int_{0}^{t}\left\langle\nu_{s}^{N}, g\left(s, \cdot, \nu_{s}^{*}, L_{s}^{N}\right) \phi-g\left(s, \cdot, \nu_{s}^{N}, L_{s}^{N}\right) \phi\right\rangle d s\right| \\
& +\mathbb{E}\left|\int_{0}^{t}\left\langle\nu_{s}^{N}, g\left(s, \cdot, \nu_{s}^{*}, L_{s}^{*}\right) \phi-g\left(s, \cdot, \nu_{s}^{*}, L_{s}^{N}\right) \phi\right\rangle d s\right| \\
= & : \mathbb{E} I_{1}^{N}+\mathbb{E} I_{2}^{N}+\mathbb{E} I_{3}^{N} .
\end{aligned}
$$

Starting with $I_{1}$, fix $\delta>0$ and recall $|g| \leq|x|+C\left(\nu^{*}\right)$ with $C\left(\nu^{*}\right) \lesssim 1+\sup _{s \leq t}\left|M_{s}^{*}\right|$. Hence, using that $\phi \in \mathscr{S}$, we can take $\lambda=\lambda(\delta)$ sufficiently large so that

$$
\sup _{x \in \mathbb{R} \backslash[-\lambda, \lambda]}\left|g\left(s, x, \nu_{s}^{*}, L_{s}^{*}\right) \phi(x)\right|<\left(1+C\left(\nu^{*}\right)\right) \delta / 2 \quad \forall s \in[0, T] .
$$

Now take a family of mollifiers $\psi_{\varepsilon} \in \mathcal{C}_{c}^{\infty}(\mathbb{R})$ and consider the (random) mollifications

$$
g_{s}^{\varepsilon}(x):=\left(g\left(s, \cdot, \nu_{s}^{*}, L_{s}^{*}\right) * \psi_{\varepsilon}\right)(x), \quad \varepsilon>0 .
$$

As $\nu^{*}$ and $\nu^{N}$ are sub-probability measures, we have

$$
I_{1} \leq\left|\int_{0}^{t}\left\langle\nu_{s}^{*}-\nu_{s}^{N}, g_{s}^{\varepsilon} \phi\right\rangle d s\right|+2\|\phi\|_{\infty} \int_{0}^{t} \sup _{|x| \leq \lambda}\left|g_{s}^{\varepsilon}-g_{s}\right| d s+\left(1+C\left(\nu^{*}\right)\right) \delta t .
$$

Since $g_{s}^{\varepsilon} \rightarrow g_{s}$ uniformly in $x \in[\lambda, \lambda]$, dominated convergence implies that, for $\varepsilon$ sufficiently small, the second term is less than $\delta$ in expectation. Likewise, $g_{s}^{\varepsilon} \phi \in \mathscr{S}$ ensures that, for $N$ large, the first term is less than $\delta$ in expectation. Hence $\mathbb{E} I_{1}^{N} \leq C(\delta+\delta+\delta t)$ for large enough $N$, where $C$ only depends on $\mathbb{E} C\left(\nu^{*}\right)$, so $\mathbb{E} I_{1}^{N} \rightarrow 0$ as $N \rightarrow \infty$.

For $I_{2}^{N}$, the local Lipschitzness of $g$, together with $\phi \in \mathscr{S}$ and $\nu_{s}^{N} \in \mathbf{M}_{\leq 1}$, gives

$$
I_{2}^{N} \leq C\left(\nu^{*}\right) \int_{0}^{t} \sup \left\{\left|\left\langle\nu_{s}^{*}-\nu_{s}^{N}, \psi\right\rangle\right|: \psi \in \mathcal{C}_{d_{0}}\right\} d s,
$$

where $\mathcal{C}_{d_{0}}:=\left\{\psi \in \mathcal{C}(\mathbb{R}):\|\psi\|_{\text {Lip }} \leq 1,|\psi(x)| \leq 1+|x|\right\}$, and $C\left(\nu^{*}\right) \lesssim 1+\sup _{s \leq t}\left|M_{s}^{*}\right|$. Fix $\delta>0$ and take $\lambda=\lambda(\delta)$ large (to be determined later). By the Arzelà-Ascoli theorem, there is a finite family $\psi_{1}, \ldots, \psi_{k} \in \mathcal{C}_{d_{0}}$ supported in $[-\lambda, \lambda]$ so that, for each $\psi \in \mathcal{C}_{d_{0}}$,

$$
\sup \left\{\left|\psi(x)-\psi_{i}(x)\right|: x \in[-\lambda, \lambda]\right\}<\delta / 2
$$


for some $i \in\{1, \ldots, k\}$. Fixing any $\psi \in \mathcal{C}_{d_{0}}(\mathbb{R})$ and the corresponding $\psi_{i}$, we have

$$
\left|\left\langle\nu^{*}-\nu^{N}, \psi\right\rangle\right| \leq\left|\int_{0}^{\lambda}\left(\psi-\psi_{i}\right) d\left(\nu^{*}-\nu^{N}\right)\right|+\left|\int_{\lambda}^{\infty}\left(\psi-\psi_{i}\right) d\left(\nu^{*}-\nu^{N}\right)\right|+\left|\left\langle\nu^{*}-\nu^{N}, \psi_{i}\right\rangle\right| .
$$

By construction, the first term is bounded by $\delta$ uniformly in $N \geq 1$. Moreover, by CauchySchwartz and Jensen's inequality, we have

$$
\mathbb{E}\left[C\left(\nu^{*}\right) \int_{0}^{t}\left|\int_{\lambda}^{\infty}\left(\psi-\psi_{i}\right) d\left(\nu_{s}^{*}-\nu_{s}^{N}\right)\right| d s\right] \leq C \mathbb{E} \int_{0}^{t}\left\langle\nu_{s}^{*}+\nu_{s}^{N},|\cdot|^{2} \mathbf{1}_{[\lambda, \infty)}\right\rangle d s
$$

and hence

$$
\mathbb{E} I_{2}^{N} \leq C \delta t+C \mathbb{E} \int_{0}^{t}\left\langle\nu_{s}^{*}+\nu_{s}^{N},|\cdot|^{2} \mathbf{1}_{[\lambda, \infty)}\right\rangle d s+C \sup _{i=1, \ldots, k} \mathbb{E} \int_{0}^{t}\left|\left\langle\nu_{s}^{*}-\nu_{s}^{N}, \psi_{i}\right\rangle\right| d s .
$$

By Corollary 3.4 and Proposition 4.3 , Lemma A.3 gives that the middle term vanishes uniformly in $N \geq 1$ as $\lambda \rightarrow \infty$, so we can take $\lambda$ large enough so that it is uniformly bounded by $\delta$. For the final term, recall that $\psi_{i}$ has compact support in $[-\lambda, \lambda]$, so we can use the same mollification argument as for $I_{1}^{N}$. Since there are only finitely many $\psi_{i}$ 's to consider, we can thus take $N$ sufficiently large such that $\mathbb{E} I_{2}^{N} \leq C(\delta t+\delta+2 \delta)$, where $C$ is a fixed numerical constant. This proves that $\mathbb{E} I_{2}^{N}$ vanishes as $N \rightarrow \infty$.

Finally, we consider the last integral $I_{3}^{N}$. By (iii) of Assumption 2.1, we have

$$
\left|\left\langle\nu_{s}^{N}, g\left(s, \cdot, \nu_{s}^{*}, \ell_{s}^{*}\right) \phi-g\left(s, \cdot, \nu_{s}^{*}, \ell_{s}^{N}\right) \phi\right\rangle\right| \lesssim C\left(\nu^{*}\right)\left|\ell_{s}^{*}-\ell_{s}^{N}\right|
$$

whenever $\ell_{s}^{*}, \ell_{s}^{N} \in\left[\theta_{i-1}, \theta_{i}\right)$ for some $i=1, \ldots, k$. Let $\left\{\ell^{*},\left(\ell^{N}\right)_{N \geq 1}\right\}$ represent a fixed realization of $\left\{L^{*},\left(L^{N}\right)_{N \geq 1}\right\}$. Then $\ell^{N} \rightarrow \ell^{*}$ in $\left(D_{\mathbb{R}}, \mathrm{M} 1\right)$ and hence $\ell_{s}^{N} \rightarrow \ell_{s}^{*}$ for any fixed $s \in\left\{r \in[0, t]: \ell_{r-}^{*}=\ell_{r}^{*}\right\}$. Fix $\varepsilon>0$. Since $\ell^{*}$ is strictly increasing (by Assumption 2.3), we can take $\delta=\delta(\varepsilon)$ small so that $\operatorname{Leb}\left(\left\{r \in[0, T]:\left|\theta_{i}-\ell_{r}^{*}\right|<\delta\right.\right.$ for some $\left.\left.i\right\}\right) \leq \varepsilon$. On the other hand, if $\left|\theta_{i}-\ell_{s}^{*}\right| \geq \delta$ for all $i$, then we eventually have $\ell_{s}^{*}, \ell_{s}^{N} \in\left[\theta_{i-1}, \theta_{i}\right)$ for some $i$, so 4.11 applies. Thus, for the given realization of the randomness,

$$
\limsup _{N \rightarrow \infty} I_{3}^{N} \leq C^{\prime}\left(\nu^{*}\right) \int_{0}^{t} \mathbf{1}_{\left\{r \in[0, T]:\left|\theta_{i}-\ell_{r}^{*}\right|<\delta \text { for some } i\right\}}(s) d s \leq C^{\prime}\left(\nu^{*}\right) \varepsilon .
$$

As $\varepsilon>0$ was arbitrary, we deduce that $\lim _{N} I_{3}^{N}=0$ almost surely. Noting the uniformity of the bound in 4.11), dominated convergence gives $\mathbb{E} I_{3}^{N} \rightarrow 0$ as $N \rightarrow \infty$.

It remains to prove $\mathbb{E} f\left(\Phi^{N}\right) \rightarrow \mathbb{E} f\left(\Phi^{*}\right)$ as $N \rightarrow \infty$. To this end, the main points are simply that $\left|\left(\mathfrak{K}^{\prime} * L^{*}\right)_{s}\right| \leq\left\|\mathfrak{K}^{\prime}\right\|_{1}$ and $\left|\int_{0}^{t}\left(\mathfrak{K}^{\prime} *\left(L^{*}-L^{N}\right)\right)_{s} d s\right| \leq\left\|\mathfrak{K}^{\prime}\right\|_{1} \int_{0}^{t}\left|L_{s}^{*}-L_{s}^{N}\right| d s$. Using these observations, the arguments are the same as for $\Psi$.

Proposition 4.8 (Martingale argument). Fix an arbitrary $\phi \in \mathscr{C}_{0}$ and define, for all $(\zeta, \ell, w) \in D_{\mathscr{S},} \times D_{\mathbb{R}} \times C_{\mathbb{R}}$, the $D_{\mathbb{R}}$-processes

$$
\begin{aligned}
& \mathcal{M}_{t}(\zeta, \ell):=\left\langle\zeta_{t}, \phi\right\rangle-\left\langle\nu_{0}, \phi\right\rangle-\int_{0}^{t}\left\langle\zeta_{s}, b\left(s, \cdot, \zeta_{s}, \ell_{s}\right) \partial_{x} \phi\right\rangle d s \\
&+ \frac{1}{2} \int_{0}^{t}\left\langle\zeta_{s}, \sigma(s, \cdot)^{2} \partial_{x x} \phi\right\rangle d s-\int_{0}^{t}\left\langle\zeta_{s}, \alpha\left(s, \cdot, \zeta_{s}, \ell_{s}\right) \partial_{x} \phi\right\rangle d(\mathfrak{K} * \ell)_{s}, \\
& \mathcal{N}_{t}(\zeta, \ell):=\mathcal{M}_{t}(\zeta, \ell)^{2}-\int_{0}^{t}\left\langle\zeta_{s}, \sigma(s, \cdot) \rho\left(s, \zeta_{s}, \ell_{s}\right) \partial_{x} \phi\right\rangle^{2} d s \\
& \mathcal{K}_{t}(\zeta, \ell, w):=\mathcal{M}_{t}(\zeta, \ell) \cdot w_{t}-\int_{0}^{t}\left\langle\zeta_{s}, \sigma(s, \cdot) \rho\left(s, \zeta_{s}, \ell_{s}\right) \partial_{x} \phi\right\rangle d s . \\
& \text { If }\left(\nu^{N}, L^{N}, W^{0}\right) \Rightarrow\left(\nu^{*}, L^{*}, W^{0}\right), \text { then } \mathcal{M}\left(\nu^{*}, L^{*}\right), \mathcal{N}\left(\nu^{*}, L^{*}\right), \text { and } \mathcal{K}\left(\nu^{*}, L^{*}, W^{0}\right) \text { are all }
\end{aligned}
$$
continuous martingales. 
Proof. Let $\mathcal{M}_{t}^{N}:=\mathcal{M}_{t}\left(\nu^{N}, L^{N}\right)$ and $\mathcal{M}_{t}^{*}:=\mathcal{M}_{t}\left(\nu^{*}, L^{*}\right)$. Now fix $s, t \in[0, T]$ with $s<t$ and define, for any $s_{1}, \ldots, s_{n} \in[0, s]$,

$$
F_{\mathcal{M}}^{N}:=\left(\mathcal{M}_{t}^{N}-\mathcal{M}_{s}^{N}\right) \prod_{i=1}^{n} f_{i}\left(\mathcal{M}_{s_{i}}^{N}\right) \quad \text { and } \quad F_{\mathcal{M}}^{*}:=\left(\mathcal{M}_{t}^{*}-\mathcal{M}_{s}^{*}\right) \prod_{i=1}^{n} f_{i}\left(\mathcal{M}_{s_{i}}^{*}\right),
$$

where $f_{1}, \ldots, f_{n} \in C_{b}(\mathbb{R})$ are arbitrary. Proceeding analogously for $\mathcal{N}$ and $\mathcal{K}$, it follows from Proposition 4.7 and the continuous mapping theorem that

$$
F_{\mathcal{M}}^{N} \Rightarrow F_{\mathcal{M}}^{*}, \quad F_{\mathcal{N}}^{N} \Rightarrow F_{\mathcal{N}}^{*}, \quad \text { and } \quad F_{\mathcal{K}}^{N} \Rightarrow F_{\mathcal{K}}^{*}
$$

Using this, and appealing to the finite dimensional evolution equation in Proposition 3.2 . the goal is now to show that

$$
\mathbb{E} F_{\mathcal{M}}^{*}=\lim _{N \rightarrow \infty} \mathbb{E} F_{\mathcal{M}}^{N}=0, \quad \mathbb{E} F_{\mathcal{N}}^{*}=\lim _{N \rightarrow \infty} \mathbb{E} F_{\mathcal{N}}^{N}=0, \quad \mathbb{E} F_{\mathcal{K}}^{*}=\lim _{N \rightarrow \infty} \mathbb{E} F_{\mathcal{K}}^{N}=0,
$$

thus proving that $\mathcal{M}^{*}, \mathcal{N}^{*}$, and $\mathcal{K}^{*}$ are true martingales, by a standard monotone class argument. Relying on uniform integrability to conclude the convergence of the means, this follows easily by minor modifications of the arguments in [35, Prop. 5.11].

By [43, Thm. 3.2] and the tightness from Propositions 4.2 and 4.5 , we can extract a weakly convergent subsequence $\left(\nu^{N}, L^{N}, W^{0}\right) \Rightarrow\left(\nu^{*}, L^{*}, W^{0}\right)$. In turn, Proposition 4.8 and the Doob-Meyer decomposition theorem allows us to conclude that, for each $\phi \in \mathscr{S}$,

$$
\begin{aligned}
& \left\langle\mathcal{M}\left(\nu^{*}, L^{*}\right)\right\rangle_{t}=\int_{0}^{t}\left\langle\nu_{s}^{*}, \sigma(s, \cdot) \rho\left(s, \nu_{s}^{*}, L_{s}^{*}\right) \partial_{x} \phi\right\rangle^{2} d s \text { and } \\
& \left\langle\mathcal{M}\left(\nu^{*}, L^{*}\right), W^{0}\right\rangle_{t}=\int_{0}^{t}\left\langle\nu_{s}^{*}, \sigma(s, \cdot) \rho\left(s, \nu_{s}^{*}, L_{s}^{*}\right) \partial_{x} \phi\right\rangle d s,
\end{aligned}
$$

so it holds for all $t \in[0, T]$ that

$$
\left\langle\mathcal{M}\left(\nu^{*}, L^{*}\right)-\int_{0}^{*}\left\langle\nu_{s}^{*}, \sigma(s, \cdot) \rho\left(s, \nu_{s}^{*}, L_{s}^{*}\right) \partial_{x} \phi\right\rangle d W_{s}^{0}\right\rangle_{t}=0 .
$$

Hence $\left(\nu^{*}, W^{0}\right)$ satisfies the SPDE 2.3 and thus the proof of Theorem 2.4 is complete.

\section{$5 \quad$ Uniqueness arguments}

In this section we present a proof of Theorem 2.6. In view of Section 4.2 we fix a limit point $\nu$ of the sequence of empirical measures $\left(\nu^{N}\right)$ and let $\tilde{\nu}$ denote another candidate solution to the SPDE (2.3). Then the strategy is to establish an energy estimate in $H^{-1}$ for the difference $\Delta_{t}:=\nu_{t}-\tilde{\nu}_{t}$, where $H^{-1}$ is the usual dual space of $H_{0}^{1}=\mathcal{W}_{0}^{1,2}\left(\mathbb{R}_{+}\right)$.

More specifically, we will rely on a 'smoothed' $H^{-1}$ estimate given in Proposition 5.1 Based on this estimate, we derive the uniqueness of the SPDE in Section 5.1.1. and then Sections 5.2 and 5.3 are devoted to the proof of Proposition 5.1 .

\subsection{Energy estimates and smoothing}

Rather than estimating the $H^{-1}$ norm of $\Delta_{t}$ directly, our approach relies on smoothing the solutions $\nu$ and $\tilde{\nu}$ by means of convolution (with a family of kernels approximating the identity). In this way, we can manipulate the resulting equations classically. Since our 
problem is phrased on the positive half-line with absorption at the boundary, it is natural to consider the family of Dirichlet heat kernels $G_{\varepsilon}$ given by

$$
G_{\varepsilon}(x, y):=p_{\varepsilon}(x-y)-p_{\varepsilon}(x+y), \quad p_{\varepsilon}(x)=(2 \pi \varepsilon)^{-\frac{1}{2}} \exp \left\{-x^{2} / 2 \varepsilon\right\} .
$$

We denote the action of $G_{\varepsilon}$ on a measure $\mu$ by $\mathcal{T}_{\varepsilon} \mu$, that is

$$
\left(\mathcal{T}_{\varepsilon} \mu\right)(x):=\int_{0}^{\infty} G_{\varepsilon}(x, y) d \mu(y) .
$$

For simplicity of presentation, we introduce the notation $\partial_{x}^{-1}$ for the anti-derivative, which amounts to

$$
\left(\partial_{x}^{-1} \mathcal{T}_{\varepsilon} \Delta_{t}\right)(x):=-\int_{x}^{\infty}\left(\mathcal{T}_{\varepsilon} \Delta_{t}\right)(y) d y
$$

Recall the embedding $\mathbf{M}_{ \pm} \hookrightarrow H^{-1}$, where $\mathbf{M}_{ \pm}$is the space of finite signed measures on $\mathbb{R}$ (with total variation norm). As in [35, Prop. 6.5], we then have

$$
\left\|\Delta_{t}\right\|_{H^{-1}} \leq \liminf _{\varepsilon \rightarrow 0}\left\|\partial_{x}^{-1} \mathcal{T}_{\varepsilon} \Delta_{t}\right\|_{2},
$$

where $\|\cdot\|_{2}$ denotes the $L^{2}$-norm on $\mathbb{R}_{+}$. Therefore, we can estimate the $H^{-1}$ norm of the difference $\Delta_{t}$ via the anti-derivatives of the smoothed solutions.

Let us now briefly outline the key ideas behind our approach. The first observation is that $y \mapsto G_{\varepsilon}(x, y)$ is an admissible test function in $\mathscr{C}_{0}$, so we can plug it into the SPDE and thus obtain expressions for the smoothed solutions, $\mathcal{T}_{\varepsilon} \nu_{t}$ and $\mathcal{T}_{\varepsilon} \tilde{\nu}_{t}$. Integrating these to introduce the anti-derivatives, and looking at their difference, this then allows us to obtain an equation for $\partial_{x}^{-1} \mathcal{T}_{\varepsilon} \Delta_{t}$ in terms of $\mathcal{T}_{\varepsilon} \Delta_{t}$ along with the occurrence of $\mathcal{T}_{\varepsilon} \nu_{t}$ and some critical 'boundary effects' as well as a collection of simpler error terms.

In order to control the $L^{2}$-norm of $\partial_{x}^{-1} \mathcal{T}_{\varepsilon} \Delta_{t}$, we thus need a uniform estimate on $\mathcal{T}_{\varepsilon} \nu_{t}$, and we need to contain the boundary effects (and error terms) as $\varepsilon \rightarrow 0$. These two tasks are the subject of Section 5.2 , which then allows us to derive the aforementioned $L^{2}$ estimate for $\partial_{x}^{-1} \mathcal{T}_{\varepsilon} \Delta_{t}$ in Section 5.3. However, before any of this, we begin by showing how to derive the uniqueness of the SPDE given that the desired energy estimate holds.

\subsubsection{Uniqueness of the SPDE - Proof of Theorem 2.6}

As above, we let $\nu$ be a limit point of the particle system and suppose $\tilde{\nu}$ is another solution to the SPDE 2.3), satisfying Assumption 2.3.

Recall that the local Lipschitzness in the loss variable only holds in a piecewise fashion on the intervals $\left[\theta_{i-1}, \theta_{i}\right)$ for $i=1, \ldots, k$. This hurdle is easily overcome by the following piecewise stopping argument: Suppose we can prove uniqueness on $\left[0, t_{0}\right]$ if $L_{t}, \tilde{L}_{t} \in\left[0, \theta_{1}\right)$ for $t<t_{0}$ (see Prop. 5.1 and the ensuing arguments) and introduce the stopping times

$$
\tau_{1}:=\inf \left\{t>0: L_{t} \geq \theta_{1}\right\} \wedge T \quad \text { and } \quad \tilde{\tau}_{1}:=\inf \left\{t>0: \tilde{L}_{t} \geq \theta_{1}\right\} \wedge T .
$$

Then $L_{t}, \tilde{L}_{t} \in\left[0, \theta_{1}\right)$ for $t<\varsigma_{1}:=\tau_{1} \wedge \tilde{\tau}_{1}$, so we get uniqueness up to $\varsigma_{1}$. Note that, by the uniqueness, $L=\tilde{L}$ on $\left[0, \varsigma_{1}\right]$ and hence $\varsigma_{1}=\tau_{1}=\tilde{\tau}_{1}$. Thus, we can repeat the uniqueness arguments on $\left[\varsigma_{1}, \varsigma_{2}\right)$, where $L_{t}, \tilde{L}_{t} \in\left[\theta_{1}, \theta_{2}\right)$, by defining $\varsigma_{2}:=\tau_{2} \wedge \tilde{\tau}_{2}$ for

$$
\tau_{2}:=\inf \left\{t>\varsigma_{1}: L_{t} \geq \theta_{2}\right\} \wedge T \quad \text { and } \quad \tilde{\tau}_{2}:=\inf \left\{t>\varsigma_{1}: \tilde{L}_{t} \geq \theta_{2}\right\} \wedge T .
$$


Continuing in this way for $\varsigma_{3}, \ldots, \varsigma_{k-1}$, we get uniqueness on all of $[0, T]$, since $L_{t}$ and $\tilde{L}_{t}$ are strictly increasing (recall part (ii) of Assumption 2.3).

Below we prove uniqueness when the local Lipschitzness in $L$ holds everywhere, noting that the arguments imply uniqueness if $L$ and $\tilde{L}$ are confined to a particular piece $\left[\theta_{i-1}, \theta_{i}\right)$. Thus, in view of the above stopping argument, the next result will suffice to complete the proof of Theorem 2.6 .

Proposition 5.1 (Smoothed $H^{-1}$ estimate). Suppose the local Lipschitzness in Assumption 2.1 (iii) holds everywhere, as opposed to piecewise. Then, as $\varepsilon \downarrow 0$, we have

$$
\begin{aligned}
\mathbb{E}\left\|\partial_{x}^{-1} \mathcal{T}_{\varepsilon} \Delta_{t \wedge t_{n}}\right\|_{2}^{2}+ & c_{0} \mathbb{E} \int_{0}^{t \wedge t_{n}}\left\|\mathcal{T}_{\varepsilon} \Delta_{s}\right\|_{2}^{2} d s \leq c_{n} \mathbb{E} \int_{0}^{t \wedge t_{n}} d_{0}\left(\nu_{s}, \tilde{\nu}_{s}\right)\left\|\partial_{x}^{-1} \mathcal{T}_{\varepsilon} \Delta_{s}\right\|_{2} d s \\
& +c_{n} \mathbb{E} \int_{0}^{t \wedge t_{n}}\left|L_{s}-\tilde{L}_{s}\right|^{2}+d_{1}\left(\nu_{s}, \tilde{\nu}_{s}\right)^{2} d s+o(1)
\end{aligned}
$$

for a fixed $c_{0}>0$, and with $c_{n}$ only depending on $n$, where $\left(t_{n}\right)$ is a sequence of stopping times such that $t_{n} \uparrow T$ as $n \uparrow \infty$.

Proof. The proof is the subject of Sections 5.2 and 5.3 .

Note that at this point Grönwall already gives that $\mathbb{E} \int_{0}^{t \wedge t_{n}}\left\|\frac{d \Delta_{s}}{d x}\right\|_{2} d s$ is finite and, in particular, $\Delta_{s \wedge t_{n}}$ has a density in $L^{2}$, which we make use of below. Observe also that $|L-\tilde{L}|$ is dominated by $d_{1}(\nu, \tilde{\nu})$, however, it is included in the estimate as it shows where the above 'piecewise stopping argument' would come into play. The next lemma relates the left- and right-hand sides of the smoothed $H^{-1}$ estimate, thus opening the door for a stronger Grönwall argument that will allow us to finish the proof of Theorem 2.6.

Lemma 5.2. There exists $c>0$ such that, for all $s \leq T, \delta \in(0,1)$, and $\lambda>1$,

$$
\left\{\begin{array}{l}
d_{0}\left(\nu_{s}, \tilde{\nu}_{s}\right) \leq c \lambda\left(1+\delta^{-1}\right)\left\|\partial_{x}^{-1} \mathcal{T}_{\varepsilon} \Delta_{s}\right\|_{2}+c \delta^{\frac{1}{2}}\left\|\frac{d \Delta_{s}}{d x}\right\|_{2}+f_{s}(\lambda)+g_{s}(\varepsilon) \\
d_{1}\left(\nu_{s}, \tilde{\nu}_{s}\right) \leq c \sqrt{\lambda+\delta^{-1}}\left\|\partial_{x}^{-1} \mathcal{T}_{\varepsilon} \Delta_{s}\right\|_{2}+c \delta^{\frac{1}{2}}\left\|\frac{d \Delta_{s}}{d x}\right\|_{2}+f_{s}(\lambda)+g_{s}(\varepsilon),
\end{array}\right.
$$

as $\varepsilon \downarrow 0$. Here $\left(f_{t}(\lambda)\right)_{t \leq T}$ is a process such that, for every $a>0, \mathbb{E} \int_{0}^{T} f_{s}(\lambda)^{2} d s \leq c_{a} e^{-a \lambda}$ for some $c_{a}>0$, and $\left(g_{s}(\varepsilon)\right)_{s \leq T}$ is process such that $\mathbb{E} \int_{0}^{T} g_{s}(\varepsilon)^{2} d s=o(1)$ as $\varepsilon \downarrow 0$.

Inserting the bound for $d_{0}$ in Prop. 5.1, we get the leading term $\lambda\left(1+\delta^{-1}\right)\left\|\partial_{x}^{-1} \mathcal{T}_{\varepsilon} \Delta_{s}\right\|_{2}^{2}$, where it is crucial that $\lambda$ is not squared. For the rest of the terms coming from $d_{0}$ we can simply apply Young's inequality. After also inserting the bound for $d_{1}$, it follows that

$$
\begin{gathered}
\mathbb{E}\left\|\partial_{x}^{-1} \mathcal{T}_{\varepsilon} \Delta_{t \wedge t_{n}}\right\|_{2}^{2} \leq \delta c_{n}^{\prime} \mathbb{E} \int_{0}^{t \wedge t_{n}}\left\|\frac{d \Delta_{s}}{d x}\right\|_{2}^{2} d s-c_{0} \mathbb{E} \int_{0}^{t \wedge t_{n}}\left\|\mathcal{T}_{\varepsilon} \Delta_{s}\right\|_{2}^{2} d s \\
+c_{n}^{\prime}\left(1+\lambda+\lambda \delta^{-1}\right) \int_{0}^{t} \mathbb{E}\left\|\partial_{x}^{-1} \mathcal{T}_{\varepsilon} \Delta_{s \wedge t_{n}}\right\|_{2}^{2} d s+c_{a}^{\prime} e^{-a \lambda}+o(1),
\end{gathered}
$$

as $\varepsilon \downarrow 0$. By construction of $\mathcal{T}_{\varepsilon}$, we have $\left\|\mathcal{T}_{\varepsilon} \Delta_{s}\right\|_{2} \leq\left\|\frac{d \Delta_{s}}{d x}\right\|_{2}$ and $\left\|\mathcal{T}_{\varepsilon} \Delta_{s}\right\|_{2} \rightarrow\left\|\frac{d \Delta_{s}}{d x}\right\|_{2}$, so dominated convergence gives

$$
\mathbb{E} \int_{0}^{t \wedge t_{n}}\left\|\mathcal{T}_{\varepsilon} \Delta_{s}\right\|_{2} d s \rightarrow \mathbb{E} \int_{0}^{t \wedge t_{n}}\left\|\frac{d \Delta_{s}}{d x}\right\|_{2} d s \quad \text { as } \quad \varepsilon \downarrow 0 .
$$


Therefore, by taking $\delta:=c_{0} / 2 c_{n}^{\prime}$, the sum of the first two terms on the right-hand side of 5.4 is eventually non-positive for small $\varepsilon>0$. In turn, we can apply the integrating factor $\exp \left\{c_{n}^{\prime}\left(1+\lambda+\lambda \delta^{-1}\right) t\right\}$ to the anti-derivative term in 5.4 and deduce that

$$
\mathbb{E}\left\|\partial_{x}^{-1} \mathcal{T}_{\varepsilon} \Delta_{t \wedge t_{n}}\right\|_{2}^{2} \leq c_{a}^{\prime} c_{n}^{\prime}\left(1+\lambda+\lambda \delta^{-1}\right) e^{c_{n}^{\prime}\left(1+\lambda+\lambda \delta^{-1}\right) T} e^{-a \lambda}+o(1)
$$

as $\varepsilon \downarrow 0$. Recalling (5.3) and appealing to Fatou's lemma, it follows that

$$
\mathbb{E}\left\|\Delta_{t \wedge t_{n}}\right\|_{H^{-1}}^{2} \leq c_{a, n, T}^{\prime}\left(1+\lambda+\lambda \delta^{-1}\right) \exp \left\{\lambda\left(1+\delta^{-1}\right) T-a \lambda\right\} .
$$

Consequently, we can simply take $a:=2\left(1+\delta^{-1}\right) T$ and send $\lambda \rightarrow \infty$ to arrive at

$$
\mathbb{E}\left\|\Delta_{t \wedge t_{n}}\right\|_{H^{-1}}^{2}=0 \quad \forall t \in[0, T] .
$$

Since $n$ was arbitrary and $t_{n} \uparrow T$ as $n \uparrow \infty$, we conclude that $\nu_{t}=\tilde{\nu}_{t}$ for all $t \in[0, T]$. This completes the proof of Theorem 2.6.

Proof of Lemma 5.2. Fix $\psi \in \mathcal{C}_{d_{0}}$, where $\mathcal{C}_{d_{0}}:=\left\{f:\|f\|_{\text {Lip }} \leq 1,|f(x)| \leq 1+|x|\right\}$. Fixing also $\lambda>1$ and $\delta>0$, we can take a cut-off function $\chi \in \mathcal{C}_{c}^{\infty}(\mathbb{R})$ equal to 1 on $[\delta, \lambda-1]$ and supported in $(\delta / 2, \lambda)$ such that

$$
|\chi| \leq 1, \quad|\partial \chi| \leq C_{\chi} / \delta \text { on }[\delta / 2, \delta], \quad \text { and } \quad|\partial \chi| \leq C_{\chi} \text { on }[\lambda-1, \lambda] .
$$

Observe that $\chi \psi \in \mathcal{W}_{0}^{1, \infty}(\delta / 2, \lambda)$ with

$$
\begin{aligned}
\|\partial(\chi \psi)\|_{2}^{2} & =\int_{0}^{\delta}|\partial \chi|^{2}|\psi|^{2} d x+\int_{\lambda-1}^{\lambda}|\partial \chi|^{2}|\psi|^{2} d x+\int_{0}^{\lambda}|\chi|^{2}|\partial \psi|^{2} d x \\
& \leq C_{\chi}^{2}\left(\delta^{-1}+1\right)\|\psi\|_{L^{\infty}(0, \lambda)}^{2}+\lambda
\end{aligned}
$$

Next, we can observe that

$$
\begin{aligned}
\left|\left\langle\Delta_{s}, \psi\right\rangle\right| & \leq\left|\left\langle\Delta_{s}, \chi \psi\right\rangle\right|+\left|\left\langle\Delta_{s},(\chi-1) \psi \mathbf{1}_{[0, \delta]}\right\rangle\right|+\left\langle\nu_{s}+\tilde{\nu}_{s},|\psi| \mathbf{1}_{[\lambda-1, \infty)}\right\rangle \\
& \leq\left|\left\langle\Delta_{s}, \chi \psi\right\rangle\right|+(1+\delta) \delta^{1 / 2}\left\|\frac{d \Delta_{s}}{d x}\right\|_{2}+\left\langle\nu_{s}+\tilde{\nu}_{s},(1+|\cdot|) \mathbf{1}_{[\lambda-1, \infty)}\right\rangle
\end{aligned}
$$

Let $(\cdot, \cdot)$ be the inner product on $L^{2}$. Integration by parts and Cauchy-Schwarz gives

$$
\begin{aligned}
\left|\left\langle\Delta_{s}, \chi \psi\right\rangle\right| & \leq\left|\left(\mathcal{T}_{\varepsilon} \Delta_{s}, \chi \psi\right)\right|+\left|\left(\mathcal{T}_{\varepsilon} \Delta_{s}, \chi \psi\right)-\left\langle\Delta_{s}, \chi \psi\right\rangle\right| \\
& \leq\left\|\partial_{x}^{-1} \mathcal{T}_{\varepsilon} \Delta_{s}\right\|_{2}\left\|\partial_{x}(\chi \psi)\right\|_{2}+\left|\left(\mathcal{T}_{\varepsilon} \Delta_{s}, \chi \psi\right)-\left\langle\Delta_{s}, \chi \psi\right\rangle\right| .
\end{aligned}
$$

By the Arzelà-Ascoli theorem applied to $\left\{\chi f: f \in \mathcal{C}_{d_{0}}\right\}$, we can find a finitely family $\left\{\varphi_{i} \in \operatorname{Lip}(\mathbb{R}): i=1, \ldots, k(\delta, \lambda)\right\}$ supported in $[\delta / 2, \lambda]$ so that, for any $f \in \mathcal{C}_{d_{0}}$,

$$
\sup _{x \in \mathbb{R}}\left|\varphi_{i}(x)-\chi f(x)\right| \leq \delta^{\frac{1}{2}} \lambda^{-\frac{1}{2}}, \quad \text { for some } i=1, \ldots, k(\delta, \lambda) .
$$

Consequently, there is a $\varphi_{i}$ such that

$$
\left|\left(\mathcal{T}_{\varepsilon} \Delta_{s}, \chi \psi\right)-\left\langle\Delta_{s}, \chi \psi\right\rangle\right| \leq\left|\left(\mathcal{T}_{\varepsilon} \Delta_{s}, \varphi_{i}\right)-\left\langle\Delta_{s}, \varphi_{i}\right\rangle\right|+2 \sqrt{\delta}\left\|\frac{d \Delta_{s}}{d x}\right\|_{2}
$$


where we have used Cauchy-Schwarz with $\left\|\mathcal{T}_{\varepsilon} \Delta_{s}\right\|_{2} \leq\left\|\frac{d \Delta_{s}}{d x}\right\|_{2}$ and $\left\|\varphi_{i}-\chi \psi\right\|_{2} \leq \sqrt{\delta}$. Note that $\left(\mathcal{T}_{\varepsilon} \Delta_{s}, \varphi_{i}\right) \rightarrow\left\langle\Delta_{s}, \varphi_{i}\right\rangle$ as $\varepsilon \rightarrow 0$ (see e.g. [35, Prop. 6.4]), so defining

$$
g_{s}^{\varepsilon}=g_{s}^{\varepsilon}(\delta, \lambda):=\sup \left\{\left|\left(\mathcal{T}_{\varepsilon} \Delta_{s}, \varphi_{i}\right)-\left\langle\Delta_{s}, \varphi_{i}\right\rangle\right|: i=1, \ldots, k(\delta, \lambda)\right\},
$$

we have $\mathbb{E} \int_{0}^{T} g_{s}(\varepsilon)^{2} d s \rightarrow 0$ as $\varepsilon \rightarrow 0$ by bounded convergence. Consequently,

$$
\left|\left\langle\Delta_{s}, \chi \psi\right\rangle\right| \leq\left\|\partial_{x}^{-1} \mathcal{T}_{\varepsilon} \Delta_{s}\right\|_{2}\left\|\partial_{x}(\chi \psi)\right\|_{2}+2 \sqrt{\delta}\left\|\frac{d \Delta_{s}}{d x}\right\|_{2}+g_{s}(\varepsilon),
$$

where $g$ is as required by the lemma. Finally, combining the above, we can take suprema over $\psi$ in the function classes that define $d_{0}$ and $d_{1}$ to find that

$$
d_{0}\left(\nu_{s}, \tilde{\nu}_{s}\right) \leq c \lambda \sqrt{1+\delta^{-1}}\left\|\partial_{x}^{-1} \mathcal{T}_{\varepsilon} \Delta_{s}\right\|_{2}+c \delta^{\frac{1}{2}}\left\|\frac{d \Delta_{s}}{d x}\right\|_{2}+f_{s}(\lambda)+g_{s}(\varepsilon),
$$

and

$$
d_{1}\left(\nu_{s}, \tilde{\nu}_{s}\right) \leq c \sqrt{\lambda+\delta^{-1}}\left\|\partial_{x}^{-1} \mathcal{T}_{\varepsilon} \Delta_{s}\right\|_{2}+c \delta^{\frac{1}{2}}\left\|\frac{d \Delta_{s}}{d x}\right\|_{2}+f_{s}(\lambda)+g_{s}(\varepsilon),
$$

where $f_{s}(\lambda):=\left\langle\nu_{s}+\tilde{\nu}_{s},(1+|\cdot|) \mathbf{1}_{[\lambda-1, \infty)}\right\rangle$. Hence the proof is complete by reference to the exponential decay properties of $f_{s}(\lambda)$ as guaranteed by Lemma A.3.

\section{$5.2 L^{2}$ regularity and containment of boundary effects}

Our first task in this section is to establish a weighted $L^{2}$ estimate for $\mathcal{T}_{\varepsilon} \nu_{t}$ that is uniform in $\varepsilon>0$ and $t \in[0, T]$. This can be achieved by exploiting the extra control that we have over $\nu$ because it arises as a limit point of the particle system.

Specifically, we can use that the empirical measures $\left(\nu^{N}\right)$ are dominated by their wholespace counterparts so that, in the limit, $\nu$ is dominated by a solution to the whole-space version of the SPDE (2.3) with $M$ and $L$ still defined in terms of $\nu$. This is crucial as we can perform the $L^{2}$ estimates for the whole-space SPDE without any boundary effects. On the other hand, the estimates for the half-line only succeed because we work in the weaker space $H^{-1}$, where we can control the boundary effects solely by relying on the boundary decay from Proposition 4.3 and Assumption 2.3 (see Lemma 5.5 and Section 5.3.2).

Proposition 5.3 ( $L^{2}$ energy estimate). Let $\nu^{*}$ be a limit point of $\left(\nu^{N}\right)$. Then

$$
\mathbb{E}\left[\sup _{t \in[0, T]} \sup _{\varepsilon>0} \int_{0}^{\infty}\left(1+x^{2}\right)\left(\mathcal{T}_{\varepsilon} \nu_{t}^{*}\right)^{2} d x\right]<\infty .
$$

Proof. The rough ideas are the same as [35, Prop. 7.1], so we will refer back to this in order to avoid duplication. First, let $\bar{\nu}^{N}:=\sum_{i=1}^{N} a_{i}^{N} \delta_{X_{t}^{i}}$ and note $\nu^{N}(A) \leq \bar{\nu}^{N}(A)$ for all $A \in \mathcal{B}(\mathbb{R})$. Now, the same work as for $\left(\nu^{N}\right)$ ensures $\left(\nu^{N}, \bar{\nu}^{N}\right)$ is tight with limit points $\nu^{*} \leq \bar{\nu}^{*}$, where $\bar{\nu}^{*}$ satisfies the whole-space analogue of the SPDE (2.3) in the sense that the space of test functions is all of $\mathscr{S}$, but with $M$ and $L$ still defined in terms of $\nu^{*}$. Arguing as in Lemmas 7.2 and 7.3 of [35], to prove (5.5) it suffices to bound

$$
J_{1}:=\liminf _{\varepsilon \rightarrow 0} \mathbb{E}\left[\sup _{t \leq T} \int_{\mathbb{R}}\left(\overline{\mathcal{T}}_{\varepsilon} \bar{\nu}_{t}^{*}\right)^{2} d x\right] \quad \text { and } \quad J_{2}:=\liminf _{\varepsilon \rightarrow 0} \mathbb{E}\left[\sup _{t \leq T} \int_{\mathbb{R}} x^{2}\left(\overline{\mathcal{T}}_{\varepsilon} \bar{\nu}_{t}^{*}\right)^{2} d x\right],
$$

where the action of $\overline{\mathcal{T}_{\varepsilon}}$ is given by

$$
\left(\overline{\mathcal{T}}_{\bar{\varepsilon}} \bar{\nu}_{t}^{*}\right)(x):=\int_{\mathbb{R}} p_{\varepsilon}(x-y) d \bar{\nu}_{t}^{*}(y) .
$$


Note that $y \mapsto p_{\varepsilon}(x-y)$ is certainly not in $\mathscr{C}_{0}$, but it has rapid decay at $\pm \infty$, so it is an admissible test function for the whole-space SPDE satisfied by $\bar{\nu}^{*}$. We split the remaining parts of the proof into three steps.

Step 1 . We begin by showing that $J_{1} \lesssim \int_{0}^{\infty} V_{0}(x)^{2} d x$. To simplify things, let

$$
\mathfrak{b}_{t}(x):=b\left(t, x, \nu_{t}, L_{t}\right)-\alpha\left(t, x, \nu_{t}, L_{t}\right) \mathfrak{L}_{t}^{\prime} .
$$

Then $\mathfrak{b}_{t}$ will play the rôle of the drift $\mu_{t}$ in the proof of Proposition 7.1 from [35]. Proceeding as in [35, we can test the SPDE with $p_{\varepsilon}(\cdot-y)$ and introduce appropriate error terms in order to get a tractable expression for $\overline{\mathcal{T}}_{\varepsilon} \bar{\nu}_{t}^{*}$. Next, we can then apply Itô's formula and thus convert this into an expression for the square $\left(\overline{\mathcal{T}}_{\varepsilon} \bar{\nu}_{t}^{*}\right)^{2}$. In this way, we arrive at

$$
\begin{aligned}
d\left(\overline{\mathcal{T}}_{\varepsilon} \bar{\nu}_{t}^{*}\right)^{2}= & -2\left(\overline{\mathcal{T}}_{\varepsilon} \bar{\nu}_{t}^{*}\right)\left(\mathfrak{b}_{t} \partial_{x}\left(\overline{\mathcal{T}}_{\varepsilon} \bar{\nu}_{t}^{*}\right)-\partial_{x} \mathfrak{b}_{t} \overline{\mathcal{H}}_{t, \varepsilon}^{\mathfrak{b}}+\overline{\mathcal{E}}_{t, \varepsilon}^{\mathfrak{b}}\right) d t \\
& +\left(\overline{\mathcal{T}}_{\varepsilon} \bar{\nu}_{t}^{*}\right) \partial_{x}\left(\sigma_{t}^{2} \partial_{x}\left(\overline{\mathcal{\mathcal { T }}}_{\varepsilon} \bar{\nu}_{t}^{*}\right)-\partial_{x} \sigma_{t}^{2} \overline{\mathcal{H}}_{t, \varepsilon}^{\sigma^{2}}+\overline{\mathcal{E}}_{t, \varepsilon}^{\sigma^{2}}\right) d t \\
& -2 \rho_{t}\left(\overline{\mathcal{T}}_{\varepsilon} \bar{\nu}_{t}^{*}\right)\left(\sigma_{t} \partial_{x}\left(\overline{\mathcal{T}}_{\varepsilon} \bar{\nu}_{t}^{*}\right)-\partial_{x} \sigma_{t} \overline{\mathcal{H}}_{t, \varepsilon}^{\sigma}+\overline{\mathcal{E}}_{t, \varepsilon}^{\sigma}\right) d W_{t} \\
& +\rho_{t}^{2}\left(\sigma_{t} \partial_{x}\left(\overline{\mathcal{T}}_{\varepsilon} \bar{\nu}_{t}^{*}\right)-\partial_{x} \sigma_{t} \overline{\mathcal{H}}_{t, \varepsilon}^{\sigma}+\overline{\mathcal{E}}_{t, \varepsilon}^{\sigma}\right)^{2} d t,
\end{aligned}
$$

where the error terms $\overline{\mathcal{E}}$ and $\overline{\mathcal{H}}$ are defined in Lemma A.2 of the Appendix. Compared to [35], we must be careful with the linear growth of $\mathfrak{b}_{t}$, however, this is taken care of via integration by parts: Since $\overline{\mathcal{T}}_{\varepsilon} \bar{\nu}_{t}^{*}$ vanishes at $\pm \infty$ by the tails of $\bar{\nu}_{t}^{*}$ (using the the whole-space analogue of Lemma A.4, we thus get

$$
\begin{aligned}
-\int_{\mathbb{R}} \int_{0}^{t} 2 \mathfrak{b}_{s}\left(\overline{\mathcal{T}}_{\varepsilon} \bar{\nu}_{s}^{*}\right) \partial_{x}\left(\overline{\mathcal{T}}_{\varepsilon} \bar{\nu}_{s}^{*}\right) d s d x & =-\int_{0}^{t} \int_{\mathbb{R}} \mathfrak{b}_{s} \partial_{x}\left(\overline{\mathcal{T}}_{\varepsilon} \bar{\nu}_{s}^{*}\right)^{2} d x d s \\
& =\int_{0}^{t} \int_{\mathbb{R}} \partial_{x} \mathfrak{b}_{s}\left(\overline{\mathcal{T}}_{\varepsilon} \bar{\nu}_{s}^{*}\right)^{2} d x d s \leq C \int_{0}^{t}\left\|\overline{\mathcal{T}}_{\bar{\varepsilon}} \bar{\nu}_{s}^{*}\right\|_{2}^{2} d s .
\end{aligned}
$$

Given this, the plan is to integrate over $x \in \mathbb{R}$ in the equation $(5.6)$ in order to obtain an estimate for the $L^{2}$-norm of $\overline{\mathcal{T}}_{\varepsilon} \bar{\nu}_{t}^{*}$. After integrating over $x$, we can then appeal to the previous estimate (5.7) and, similarly, we can perform another integration by parts in the second line on the right-hand side of $(5.6)$. Using this, and the fact that $\left|\overline{\mathcal{H}}_{t, \varepsilon}^{g}\right| \lesssim \overline{\mathcal{T}}_{2 \varepsilon} \bar{\nu}_{t}^{*}$ by Lemma A.2. we can thus apply Young's inequality (with free parameter $\theta>0$ ) to see that

$$
\begin{aligned}
\left\|\overline{\mathcal{T}}_{\varepsilon} \bar{\nu}_{t}^{*}\right\|_{2}^{2} \leq & \left\|\overline{\mathcal{T}}_{\varepsilon} \nu_{0}\right\|_{2}^{2}+C_{\theta} \int_{0}^{t}\left\|\overline{\mathcal{T}}_{\varepsilon} \bar{\nu}_{s}^{*}\right\|_{2}^{2} d s+C_{\theta} \int_{0}^{t}\left\|\overline{\mathcal{T}}_{2 \varepsilon} \bar{\nu}_{s}^{*}\right\|_{2}^{2} d s \\
& +C_{\theta} \int_{0}^{t}\left(\left\|\overline{\mathcal{E}}_{s, \varepsilon}^{\mathfrak{b}}\right\|_{2}^{2}+\left\|\overline{\mathcal{E}}_{s, \varepsilon}^{\sigma^{2}}\right\|_{2}^{2}+\left\|\overline{\mathcal{E}}_{s, \varepsilon}^{\sigma}\right\|_{2}^{2}\right) d s \\
& +\int_{0}^{t} \int_{\mathbb{R}}\left(\rho_{s}^{2} \sigma_{s}^{2}+\theta \rho_{s}^{2} \sigma_{s}^{2}-\sigma_{s}^{2}+\theta\right)\left|\partial_{x}\left(\overline{\mathcal{T}}_{\varepsilon} \bar{\nu}_{s}^{*}\right)\right|^{2} d x d s \\
& +\int_{0}^{t} \int_{\mathbb{R}} \rho_{s} \partial_{x} \sigma_{s}\left(\overline{\mathcal{T}}_{\varepsilon} \bar{\nu}_{s}^{*}\right)^{2}+2 \rho_{s}\left(\overline{\mathcal{T}}_{\varepsilon} \bar{\nu}_{s}^{*}\right)\left(\partial_{x} \sigma_{s}^{2} \overline{\mathcal{H}}_{s, \varepsilon}^{\sigma}-\overline{\mathcal{E}}_{s, \varepsilon}^{\sigma}\right) d x d W_{s} .
\end{aligned}
$$

Here we have used the stochastic fubini theorem to switch the order of integration in the stochastic integral (allowed due to the exponential tails as in [35, Lem. 8.3]) and we have also integrated by parts in the $d x$-integral inside the stochastic integral. 
Step 2. Since $\rho^{2}$ is bounded away from 1 (and $\sigma^{2}$ is bounded away from 0), we can choose $\theta$ sufficiently small so that the third line of $(5.8)$ is negative and hence we can discard it. Raising both sides of $(5.8)$ to a power $k \geq 1$, we thus have

$$
\begin{aligned}
\sup _{r \leq t}\left\|\overline{\mathcal{T}}_{\varepsilon} \bar{\nu}_{r}^{*}\right\|_{2}^{2 k} \leq & C\left\|\overline{\mathcal{T}}_{\varepsilon} \nu_{0}\right\|_{2}^{2 k}+C \int_{0}^{t}\left(\left\|\overline{\mathcal{T}}_{\varepsilon} \bar{\nu}_{s}^{*}\right\|_{2}^{2 k}+\left\|\overline{\mathcal{T}}_{2 \varepsilon} \bar{\nu}_{s}^{*}\right\|_{2}^{2 k}\right) d s \\
& +C \int_{0}^{t}\left(\left\|\overline{\mathcal{E}}_{s, \varepsilon}^{\mathfrak{b}}\right\|_{2}^{2 k}+\left\|\overline{\mathcal{E}}_{s, \varepsilon}^{\sigma^{2}}\right\|_{2}^{2 k}+\left\|\overline{\mathcal{E}}_{s, \varepsilon}^{\sigma}\right\|_{2}^{2 k}\right) d s \\
& +C\left\{\sup _{r \leq t} \int_{0}^{r} \int_{\mathbb{R}} \rho_{s} \partial_{x} \sigma_{s}\left(\overline{\mathcal{T}}_{\varepsilon} \bar{\nu}_{s}^{*}\right)^{2}+2 \rho_{s}\left(\overline{\mathcal{T}}_{\varepsilon} \bar{\nu}_{s}^{*}\right)\left(\partial_{x} \sigma_{s}^{2} \overline{\mathcal{H}}_{s, \varepsilon}^{\sigma}-\overline{\mathcal{E}}_{s, \varepsilon}^{\sigma}\right) d x d W_{s}\right\}^{k},
\end{aligned}
$$

with $C=C(k)$. By Burkholder-Davis-Gundy, and Hölder's and Young's inequalities, the expected supremum of the stochastic integral is bounded by a constant times

$$
\begin{aligned}
\mathbb{E}\left\{\int_{0}^{t}\left(\int_{\mathbb{R}}\left(\overline{\mathcal{T}}_{\varepsilon} \bar{\nu}_{s}^{*}\right)\left(\overline{\mathcal{T}}_{\varepsilon} \bar{\nu}_{s}^{*}+\left|\partial_{x} \sigma_{s}^{2} \overline{\mathcal{H}}_{s, \varepsilon}^{\sigma}\right|+\left|\overline{\mathcal{E}}_{s, \varepsilon}^{\sigma}\right|\right) d x\right)^{2} d s\right\}^{k / 2} \\
\leq C^{\prime} \mathbb{E} \sup _{r \leq t}\left\|\overline{\mathcal{T}}_{\varepsilon} \bar{\nu}_{r}^{*}\right\|_{2}^{2 k}+C^{\prime \prime} \mathbb{E} \int_{0}^{t}\left(\left\|\overline{\mathcal{T}}_{\varepsilon} \bar{\nu}_{t}^{*}\right\|_{2}^{2 k}+\left\|\overline{\mathcal{T}}_{2 \varepsilon} \bar{\nu}_{t}^{*}\right\|_{2}^{2 k}+\left\|\overline{\mathcal{E}}_{s, \varepsilon}^{\sigma}\right\|_{2}^{2 k}\right) d s,
\end{aligned}
$$

Consequently, taking expectations in $(5.9)$ gives

$$
\begin{aligned}
\inf _{\varepsilon \leq \varepsilon^{\prime}} \mathbb{E} \sup _{r \leq t}\left\|\overline{\mathcal{T}}_{\varepsilon} \bar{\nu}_{r}^{*}\right\|_{2}^{2 k} \leq & C_{0} \inf _{\varepsilon \leq \varepsilon^{\prime}}\left\|\overline{\mathcal{T}}_{\varepsilon} \nu_{0}\right\|_{2}^{2 k}+t C_{0} \inf _{\varepsilon \leq \varepsilon^{\prime}} \mathbb{E} \sup _{r \leq t}\left\|\overline{\mathcal{T}}_{\varepsilon} \bar{\nu}_{r}^{*}\right\|_{2}^{2 k} \\
& +C_{0} \inf _{\varepsilon \leq \varepsilon^{\prime}} \mathbb{E} \int_{0}^{t}\left(\left\|\overline{\mathcal{E}}_{s, \varepsilon}^{\mathfrak{b}}\right\|_{2}^{2 k}+\left\|\overline{\mathcal{E}}_{s, \varepsilon}^{\sigma^{2}}\right\|_{2}^{2 k}+\left\|\overline{\mathcal{E}}_{s, \varepsilon}^{\sigma}\right\|_{2}^{2 k}\right) d s .
\end{aligned}
$$

If we now restrict to $t \leq T_{0}:=1 / 2 C_{0}$ and send $\varepsilon^{\prime} \rightarrow 0$, then we get

$$
\liminf _{\varepsilon \rightarrow 0} \mathbb{E} \sup _{t \leq T_{0}}\left\|\overline{\mathcal{T}}_{\varepsilon} \bar{\nu}_{t}^{*}\right\|_{2}^{2 k} \leq 2 C_{0}\left\|V_{0}\right\|_{2}^{2 k},
$$

where we have used that $\left\|\overline{\mathcal{T}}_{\varepsilon} \nu_{0}\right\|_{2} \rightarrow\left\|V_{0}\right\|_{2}$ as $\varepsilon \rightarrow 0$ and that the error terms vanish by Lemma A.2. For $k=1$, this proves the bound on $J_{1}$ in small time, that is, for $t \in\left[0, T_{0}\right]$ with $T_{0}=1 / 2 C_{0}$. The extension of this bound to all of $[0, T]$ follows by propagating the argument onto the finitely many intervals $\left[T_{k}, T_{k+1} \wedge T\right]$ with $T_{k+1}=T_{k}+1 / 2 C_{0}$ for $k=1, \ldots,\left\lceil 2 C_{0} T\right\rceil$ (as in the proof of [35, Prop. 7.1]).

Step 3. We now show how to extend the previous work to prove that $J_{2}$ is finite. In order to succeed at this, we will need to control the 4th moments of $\sup _{t \leq T}\left\|\overline{\mathcal{T}}_{\varepsilon} \bar{\nu}_{t}^{*}\right\|_{2}$, which is the reason for introducing the power $k$ in Step 2 above. The idea is simply to multiply by $x^{2}$ in $(5.6)$ before integrating over $x \in \mathbb{R}$ and then proceed as in Steps 1 and 2 above. Beginning with the first term, an integration by part yields

$$
\begin{aligned}
-\int_{\mathbb{R}} \int_{0}^{t} 2 x^{2} \mathfrak{b}_{s}\left(\overline{\mathcal{T}}_{\varepsilon} \bar{\nu}_{s}^{*}\right) \partial_{x}\left(\overline{\mathcal{T}}_{\varepsilon} \bar{\nu}_{s}^{*}\right) d s d x & =\int_{0}^{t} \int_{\mathbb{R}}\left(x^{2} \partial_{x} \mathfrak{b}_{s}+2 x \mathfrak{b}_{s}\right)\left(\overline{\mathcal{T}}_{\varepsilon} \bar{\nu}_{s}^{*}\right)^{2} d x d s \\
& \leq C \int_{0}^{t}\left\|x\left(\overline{\mathcal{T}}_{\varepsilon} \bar{\nu}_{s}^{*}\right)\right\|_{2}^{2} d s+C \int_{0}^{t}\left(1+\left|M_{s}\right|\right)\left\|\overline{\mathcal{T}}_{\varepsilon} \bar{\nu}_{s}^{*}\right\|_{2}^{2} d s
\end{aligned}
$$


Crucially, the second term on the right-hand side can be controlled by

$$
\mathbb{E} \int_{0}^{t}\left(1+\left|M_{s}\right|\right)\left\|\overline{\mathcal{T}}_{\varepsilon} \bar{\nu}_{s}^{*}\right\|_{2}^{2} d s \leq \int_{0}^{t} \mathbb{E}\left[\left(1+\left|M_{s}\right|\right)^{2}\right] d s+\int_{0}^{t} \mathbb{E}\left[\left\|\overline{\mathcal{T}}_{\varepsilon} \bar{\nu}_{s}^{*}\right\|_{2}^{4}\right] d s,
$$

which is finite because of the result in Step 2 with $k=2$. Arguments completely analogous to those that led to $5.8-(5.9)$ then yield

$$
\begin{aligned}
\left\|x \overline{\mathcal{T}}_{\varepsilon} \bar{\nu}_{t}^{*}\right\|_{2}^{2} \leq & \left\|x \overline{\mathcal{T}}_{\varepsilon} \nu_{0}\right\|_{2}^{2}+C_{\theta} \int_{0}^{t}\left\|x \overline{\mathcal{T}}_{\varepsilon} \bar{\nu}_{s}^{*}\right\|_{2}^{2} d s+C_{\theta} \int_{0}^{t}\left\|x \overline{\mathcal{T}}_{2 \varepsilon} \bar{\nu}_{s}^{*}\right\|_{2}^{2} d s+C^{\prime} \\
& +C_{\theta} \int_{0}^{t}\left(\left\|x \overline{\mathcal{E}}_{s, \varepsilon}^{\mathfrak{b}}\right\|_{2}^{2}+\left\|x \overline{\mathcal{E}}_{s, \varepsilon}^{\sigma^{2}}\right\|_{2}^{2}+\left\|x \overline{\mathcal{E}}_{s, \varepsilon}^{\sigma}\right\|_{2}^{2}\right) d s \\
& -2 \int_{0}^{t} \int_{\mathbb{R}} x^{2} \rho_{s} \overline{\mathcal{T}}_{\varepsilon} \bar{\nu}_{s}^{*}\left(\sigma_{s} \partial_{x} \overline{\mathcal{T}}_{\varepsilon} \bar{\nu}_{t}^{*}+\partial_{x} \sigma_{s} \overline{\mathcal{H}}_{s, \varepsilon}^{\sigma}+\overline{\mathcal{E}}_{s, \varepsilon}^{\sigma}\right) d x d W_{s},
\end{aligned}
$$

where the extra constant $C^{\prime}$ comes from 5.10 . Given this, we can argue as in Step 2 (with $k=1$ ) to bound $J_{2}$ by a multiple of $C^{\prime}+\left\|x V_{0}\right\|_{2}^{2}$.

Corollary 5.4 (Density process). Any limit point $\nu^{*}$ of $\left(\nu^{N}\right)$ has an $L^{2}$-valued density process $\left(V_{t}^{*}\right)_{t \geq 0}$ with $\left\|x V_{t}^{*}\right\|_{2}<\infty$.

Proof. Given Proposition 5.3 , this follows by a standard weak compactness argument for the $L^{2}$-bounded sequence $\left(\mathcal{T}_{\varepsilon} \nu_{t}^{*}\right)_{\epsilon>0}$.

The next lemma represses the 'boundary effects' in the estimates for $\partial_{x}^{-1} \mathcal{T}_{\varepsilon} \Delta_{t}$, by ensuring that the relevant terms vanish as $\varepsilon \rightarrow 0$. This depends critically on the behaviour of the mass of the solutions near the boundary, so the essential ingredient is the boundary decay from Assumption 2.3 as satisfied by the limit points because of Proposition 4.3 .

Lemma 5.5 (Boundary estimate). Let $\mu$ satisfy Assumption 2.3 and let $g_{t}(y)$ be a (stochastic) function with $\left|g_{t}(y)\right| \lesssim 1+|y|+M_{t}$, where $M_{t}:=\left\langle\mu_{t}, \psi\right\rangle$ for some $\psi \in \operatorname{Lip}(\mathbb{R})$. Then

$$
\mathbb{E} \int_{0}^{T} \int_{0}^{\infty}\left|\left\langle\mu_{t}, g_{t}(\cdot) p_{\varepsilon}(x+\cdot)\right\rangle\right|^{2} d x d t \rightarrow 0 \quad \text { as } \quad \varepsilon \rightarrow 0 .
$$

Proof. Notice first that, by Jensen's inequality,

$$
\left|\left\langle\mu_{t}, g_{t}(\cdot) p_{\varepsilon}(x+\cdot)\right\rangle\right|^{2} \leq C \varepsilon^{-1} e^{-x^{2} / \varepsilon} \int_{0}^{\infty}\left(1+y^{2}+M_{t}^{2}\right) e^{-y^{2} / \varepsilon} d \mu_{t}(y) .
$$

and hence

$$
\int_{0}^{\infty}\left|\left\langle\mu_{t}, g_{t}(\cdot) p_{\varepsilon}(x+\cdot)\right\rangle\right|^{2} d x \leq C \int_{0}^{\infty}\left(1+y^{2}+M_{t}^{2}\right) \varepsilon^{-\frac{1}{2}} e^{-y^{2} / \varepsilon} d \mu_{t}(y)
$$

We divide the proof into three cases, where either $1, y^{2}$ or $M_{t}^{2}$ appears in the integrand. The first case follows as in [35, Lem. 7.6]. For the second case, fix $0<\varepsilon<1$, and let $\eta \in(0,1)$ be a free parameter. Splitting the integral on $y \leq \varepsilon^{\eta}$ and its complement gives

$$
\varepsilon^{-\frac{1}{2}} \int_{0}^{\infty} y^{2} e^{-y^{2} / \varepsilon} d \mu_{t}(y) \leq \varepsilon^{-\frac{1}{2}}\left(\mu_{t}\left(0, \varepsilon^{\eta}\right)+\left\langle\mu_{t}, y^{2}\right\rangle \exp \left\{-\varepsilon^{2 \eta-1}\right\}\right) .
$$


By (iii)-(iv) of Assumption 2.3, appealing also to Lemma A.3, it follows that

$$
\mathbb{E} \int_{0}^{T} \int_{0}^{\infty} y^{2} \varepsilon^{-\frac{1}{2}} e^{-y^{2} / \varepsilon} d \mu_{t}(y) d t \leq C \varepsilon^{-\frac{1}{2}}\left(\varepsilon^{\eta(1+\beta)}+\exp \left\{-\varepsilon^{2 \eta-1}\right\}\right)
$$

for a constant $\beta>0$, where the right-hand side of 5.13 converges to zero as long as

$$
(2+2 \beta)^{-1}<\eta<2^{-1} \text {. }
$$

For the final case, we can rely on Hölder's inequality to see that

$$
\mathbb{E} \int_{0}^{T} M_{t}^{2} \int_{0}^{\infty} \varepsilon^{-\frac{1}{2}} e^{-y^{2} / \varepsilon} d \mu_{t} d t \leq \mathbb{E}\left[\int_{0}^{T} M_{t}^{2 q} d t\right]^{\frac{1}{q}} \mathbb{E}\left[\int_{0}^{T}\left|\int_{0}^{\infty} \varepsilon^{-\frac{1}{2}} e^{-y^{2} / \varepsilon} d \mu_{t}\right|^{p} d t\right]^{\frac{1}{p}} .
$$

Since $M_{t}^{2 q} \lesssim \mu_{t}(0, \infty)+\left\langle\mu_{t}, y^{2 q}\right\rangle$ by the Lipschitzness of $\psi$, we get $\mathbb{E} \int_{0}^{T} M_{t}^{2 q} d t<\infty$ for all $q>1$ by Assumption 2.3 (iii) and Lemma A.3. Moreover, arguing as in (5.12 - 5.13),

$$
\mathbb{E} \int_{0}^{T}\left|\int_{0}^{\infty} \varepsilon^{-\frac{1}{2}} e^{-y^{2} / \varepsilon} d \mu_{t}(y)\right|^{p} d t \leq C \varepsilon^{-\frac{p}{2}}\left(\varepsilon^{\eta(1+\beta)}+\exp \left\{-p \varepsilon^{2 \eta-1}\right\}\right),
$$

so the claim follows by taking $p:=1+\beta / 2$ and choosing $\eta$ in the range

$$
(1+\beta / 2)(2+2 \beta)^{-1}<\eta<2^{-1} \text {. }
$$

This finishes the proof.

\subsection{The smoothed $H^{-1}$ estimate - proof of Proposition 5.1}

In this section we finalize the proof of the smoothed $H^{-1}$ estimate from Proposition 5.1 . Remark 5.6 ('o $o_{\mathrm{sq}}(1)$ '-notation). As in [35, we denote by $o_{\mathrm{sq}}(1)$ any family of $L^{2}$ valued processes $\left\{\left(\zeta_{t}^{\varepsilon}\right)_{t \leq T}\right\}_{\varepsilon>0}$ that satisfy $\mathbb{E} \int_{0}^{T}\left\|\zeta_{t}^{\varepsilon}\right\|_{2}^{2} d t \rightarrow 0$ as $\varepsilon \downarrow 0$. For example, Lemma 5.5 and Lemma A.1 ensure that the 'boundary effects' and error terms will be $o_{\mathrm{sq}}(1)$.

As outlined at the start of Section 5.1, we will derive an equation for $\partial_{x}^{-1} \mathcal{T}_{\varepsilon} \Delta_{t}$ by testing the SPDE for $\nu$ and $\tilde{\nu}$ with the kernel $y \mapsto G_{\varepsilon}(\cdot, y)$ and then integrating the difference of the resulting expressions over $(x, \infty)$ for $x>0$. As in the proof of Proposition 5.3, we set $\mathfrak{b}_{t}:=b_{t}-\alpha_{t} \mathfrak{L}_{t}^{\prime}$ and $\tilde{\mathfrak{b}}_{t}:=\tilde{b}_{t}-\tilde{\alpha}_{t} \tilde{\mathfrak{L}}_{t}^{\prime}$. Then we can argue as in [35, Sec. 7], using Lemma 5.5 and Lemma A.1] in place of their counterparts in [35], to conclude that

$$
\begin{aligned}
d \partial_{x}^{-1} \mathcal{T}_{\varepsilon} \Delta_{t}= & -\left(\tilde{\mathfrak{b}}_{t} \mathcal{T}_{\varepsilon} \Delta_{t}+\delta_{t}^{\mathfrak{b}} \mathcal{T}_{\varepsilon} \nu_{t}\right) d t+\frac{1}{2} \partial_{x}\left(\sigma_{t}^{2} \mathcal{T}_{\varepsilon} \Delta_{t}+\mathcal{E}_{t, \varepsilon}^{\sigma^{2}}-\tilde{\mathcal{E}}_{t, \varepsilon}^{\sigma^{2}}\right) d t \\
& -\sigma_{t}\left(\tilde{\rho} \mathcal{T}_{\varepsilon} \Delta_{t}+\delta_{t}^{\rho} \mathcal{T}_{\varepsilon} \nu_{t}\right) d W_{t}^{0}+o_{\mathrm{sq}}(1) d t+o_{\mathrm{sq}}(1) d W_{t}^{0},
\end{aligned}
$$

where $\mathcal{E}^{g}$ is an error term defined in Lemma A.1, with $\tilde{\mathcal{E}}^{g}$ defined analogously only for $\tilde{\nu}$, and

$$
\delta_{t}^{g}:=g\left(t, x, \nu_{t}, L_{t}\right)-g\left(t, x, \tilde{\nu}_{t}, \tilde{L}_{t}\right) .
$$

Applying Itô's formula, it follows that

$$
\begin{aligned}
d\left(\partial_{x}^{-1} \mathcal{T}_{\varepsilon} \Delta_{t}\right)^{2}= & -2\left(\partial_{x}^{-1} \mathcal{T}_{\varepsilon} \Delta_{t}\right)\left(\tilde{\mathfrak{b}}_{t} \mathcal{T}_{\varepsilon} \Delta_{t}+\delta_{t}^{\mathfrak{b}} \mathcal{T}_{\varepsilon} \nu_{t}\right) d t \\
& +\left(\partial_{x}^{-1} \mathcal{T}_{\varepsilon} \Delta_{t}\right) \partial_{x}\left(\sigma_{t}^{2} \mathcal{T}_{\varepsilon} \Delta_{t}+\mathcal{E}_{t, \varepsilon}^{\sigma^{2}}-\tilde{\mathcal{E}}_{t, \varepsilon}^{\sigma^{2}}\right) d t \\
& +\sigma_{t}^{2}\left(\tilde{\rho} \mathcal{T}_{\varepsilon} \Delta_{t}+\delta_{t}^{\rho} \mathcal{T}_{\varepsilon} \nu_{t}\right)^{2} d t \\
& -2 \alpha\left(\partial_{x}^{-1} \mathcal{T}_{\varepsilon} \Delta_{t}\right) \sigma_{t}\left(\tilde{\rho} \mathcal{T}_{\varepsilon} \Delta_{t}+\delta_{t}^{\rho} \mathcal{T}_{\varepsilon} \nu_{t}\right) d W_{t} \\
& +\left(\partial_{x}^{-1} \mathcal{T}_{\varepsilon} \Delta_{t}\right) o_{\mathrm{sq}}(1) d t+\left(\partial_{x}^{-1} \mathcal{T}_{\varepsilon} \Delta_{t}\right) o_{\mathrm{sq}}(1) d W_{t}+o_{\mathrm{sq}}(1)^{2} d t
\end{aligned}
$$


We can now integrate the above in $x \in \mathbb{R}_{+}$to arrive at the $L^{2}$ norm of $\partial_{x}^{-1} \mathcal{T}_{\varepsilon} \Delta_{t}$. Then it remains to estimate the resulting integrals on the right-hand side - a procedure we split into five short steps (deviating substantially from [35]). For convenience, we define

$$
|M|_{t, \star}:=\sup _{s \leq t}\left\{1+\left|M_{t}\right|+\left|\tilde{M}_{t}\right|\right\} \text { and }\|\mathcal{T} \nu\|_{t, \star}:=\sup _{s \leq t} \sup _{\varepsilon>0}\left\|(1+x) \mathcal{T}_{\varepsilon} \nu_{s}\right\|_{2} .
$$

Step 1. We start by considering the first line on the right-hand side of (5.14). Note that we can write $2\left(\partial_{x}^{-1} \mathcal{T}_{\varepsilon} \Delta_{t}\right)\left(\mathcal{T}_{\varepsilon} \Delta_{t}\right)=\partial_{x}\left(\partial_{x}^{-1} \mathcal{T}_{\varepsilon} \Delta_{t}\right)^{2}$ in the first term. Hence, we can integrate by parts in $x$ and use Young's inequality with free parameter $\theta$ to get

$$
\begin{aligned}
-\int_{0}^{\infty} 2 \tilde{\mathfrak{b}}_{t}\left(\partial_{x}^{-1} \mathcal{T}_{\varepsilon} \Delta_{t}\right)\left(\mathcal{T}_{\varepsilon} \Delta_{t}\right) d x=- & \int_{0}^{\infty}\left(\partial_{x} \tilde{\mathfrak{b}}_{t}\right)\left(\partial_{x}^{-1} \mathcal{T}_{\varepsilon} \Delta_{t}\right)^{2} d x \\
& +2 \tilde{\mathfrak{b}}_{t}(0) \int_{0}^{\infty}\left(\partial_{x}^{-1} \mathcal{T}_{\varepsilon} \Delta_{t}\right)\left(\mathcal{T}_{\varepsilon} \Delta_{t}\right) d x \\
\leq C_{\mathfrak{b}} & \left\|\partial_{x}^{-1} \mathcal{T}_{\varepsilon} \Delta_{t}\right\|_{2}^{2}+C_{\theta} C_{\mathfrak{b}}|M|_{t, \star}^{2}\left\|\partial_{x}^{-1} \mathcal{T}_{\varepsilon} \Delta_{t}\right\|_{2}^{2}+\theta\left\|\mathcal{T}_{\varepsilon} \Delta_{t}\right\|_{2}^{2}
\end{aligned}
$$

For the second integral in the first line of (5.14), we recall that

$$
\left|\delta_{t}^{\mathfrak{b}}\right| \lesssim\left(|x|+|M|_{t, \star}\right)\left(d_{0}\left(\nu_{t}, \tilde{\nu}_{t}\right)+\left|L_{t}-\tilde{L}_{t}\right|+\left|\mathfrak{L}_{t}^{\prime}-\tilde{\mathfrak{L}}_{t}^{\prime}\right|\right) .
$$

Thus, using Cauchy-Schwarz on the $d_{0}$ term and Young's inequality on the others,

$$
\begin{aligned}
& \left|\int_{0}^{\infty} \delta_{t}^{\mathfrak{b}}\left(\partial_{x}^{-1} \mathcal{T}_{\varepsilon} \Delta_{t}\right) \mathcal{T}_{\varepsilon} \nu_{t} d x\right| \lesssim|M|_{t, \star}\left\|\mathcal{T}_{\nu}\right\|_{t, \star}\left\|\partial_{x}^{-1} \mathcal{T}_{\varepsilon} \Delta_{t}\right\|_{2} d_{0}\left(\nu_{t}, \tilde{\nu}_{t}\right) \\
& \quad+|M|_{t, \star}^{2}\left\|\partial_{x}^{-1} \mathcal{T}_{\varepsilon} \Delta_{t}\right\|_{2}^{2}+|M|_{t, \star}^{2}\left\|\mathcal{T}_{\nu}\right\|_{t, \star}^{2}\left(\left|L_{t}-\tilde{L}_{t}\right|^{2}+\left|\mathfrak{L}_{t}^{\prime}-\tilde{\mathfrak{L}}_{t}^{\prime}\right|^{2}\right),
\end{aligned}
$$

where we can note that

$$
\begin{aligned}
\int_{0}^{t}|M|_{s, \star}^{2}\|\mathcal{T} \nu\|_{s, \star}^{2}\left|\mathfrak{L}_{s}^{\prime}-\tilde{\mathfrak{L}}_{s}^{\prime}\right|^{2} d s & \leq|M|_{t, \star}^{2}\|\mathcal{T} \nu\|_{t, \star}^{2} \int_{0}^{t}\left|\int_{0}^{s} \mathfrak{K}^{\prime}(s-r)\left(L_{r}-\tilde{L}_{r}\right) d r\right|^{2} d s \\
& \leq|M|_{t, \star}^{2}\|\mathcal{T} \nu\|_{t, \star}^{2}\left\|\mathfrak{K}^{\prime}\right\|_{1}^{2} \int_{0}^{t}\left|L_{r}-\tilde{L}_{r}\right|^{2} d r .
\end{aligned}
$$

Step 2. Now consider the second line on the right-hand-side of (5.14). By performing an integration by parts and using Young's inequality with free parameter $\theta$, we get

$$
\begin{array}{r}
\int_{0}^{\infty}\left(\partial_{x}^{-1} \mathcal{T}_{\varepsilon} \Delta_{t}\right) \partial_{x}\left(\sigma_{t}^{2} \mathcal{T}_{\varepsilon} \Delta_{t}+\mathcal{E}_{t, \varepsilon}^{\sigma^{2}}-\tilde{\mathcal{E}}_{t, \varepsilon}^{\sigma^{2}}\right) d x=-\left\|\sigma_{t} \mathcal{T}_{\varepsilon} \Delta_{t}\right\|_{2}^{2}-\int_{0}^{\infty}\left(\mathcal{T}_{\varepsilon} \Delta_{t}\right)\left(\mathcal{E}_{t, \varepsilon}^{\sigma^{2}}-\tilde{\mathcal{E}}_{t, \varepsilon}^{\sigma^{2}}\right) d x \\
\leq-\left\|\sigma_{t} \mathcal{T}_{\varepsilon} \Delta_{t}\right\|_{2}^{2}+C_{\theta}\left\|\mathcal{E}_{t, \varepsilon}^{\sigma^{2}}-\tilde{\mathcal{E}}_{t, \varepsilon}^{\sigma^{2}}\right\|_{2}^{2}+\theta\left\|\mathcal{T}_{\varepsilon} \Delta_{t}\right\|_{2}^{2},
\end{array}
$$

where we have used that $\mathcal{T}_{\varepsilon} \Delta_{t}, \mathcal{E}_{t, \varepsilon}^{g}$, and $\tilde{\mathcal{E}}_{t, \varepsilon}^{g}$ are zero at zero and vanish at infinity.

Step 3. In the third line on the right-hand side of (5.14), we expand the square and apply Young's inequality with free parameter $\theta$ to the lower order term. This yields

$$
\begin{aligned}
\int_{0}^{\infty} \sigma_{t}^{2}\left(\tilde{\rho} \mathcal{T}_{\varepsilon} \Delta_{t}+\delta_{t}^{\rho} \mathcal{T}_{\varepsilon} \nu_{t}\right)^{2} d x \leq & \left\|\sigma_{t} \tilde{\rho}_{t} \mathcal{T}_{\varepsilon} \Delta_{t}\right\|_{2}^{2}+C_{\sigma}\left|\delta_{t}^{\rho}\right|^{2}\left\|\mathcal{T}_{\varepsilon} \nu_{t}\right\|_{2}^{2} \\
& +C_{\theta}\left|\delta^{\rho}\right|^{2}\left\|\mathcal{T}_{\varepsilon} \nu_{t}\right\|_{2}^{2}+\theta\left\|\mathcal{T}_{\varepsilon} \Delta_{t}\right\|_{2}^{2}
\end{aligned}
$$


Also, we recall here that $\left|\delta_{t}^{\rho}\right|^{2} \lesssim|M|_{t, \star}^{2}\left(\left|L_{t}-\tilde{L}_{t}\right|^{2}+d_{1}\left(\nu_{t}, \tilde{\nu}_{t}\right)^{2}\right)$.

Step 4. When taking expectation in (5.14), the stochastic integrals vanish. Thus, by taking expectation and integrating over $x>0$, it follows from Steps 1-3 and Young's inequality with free parameter $\theta>0$ that

$$
\begin{aligned}
\mathbb{E}\left\|\partial_{x}^{-1} \mathcal{T}_{\varepsilon} \Delta_{t}\right\|_{2}^{2} \leq C_{\theta} \mathbb{E} \int_{0}^{t}\|\mathcal{T} \nu\|_{s, \star}^{2}|M|_{s, \star}^{2}\left(\left|L_{s}-\tilde{L}_{s}\right|^{2}+d_{1}\left(\nu_{s}, \tilde{\nu}_{s}\right)^{2}\right) d s \\
+C_{\theta} \mathbb{E} \int_{0}^{t}|M|_{s, \star}^{2}\left\|\partial_{x}^{-1} \mathcal{T}_{\varepsilon} \Delta_{s}\right\|_{2}^{2} d s+C_{\theta} \mathbb{E} \int_{0}^{t}|M|_{s, \star}\left\|\mathcal{T}_{\nu}\right\|_{s, \star}\left\|\partial_{x}^{-1} \mathcal{T}_{\varepsilon} \Delta_{s}\right\|_{2} d_{0}\left(\nu_{s}, \tilde{\nu}_{s}\right) d s \\
+\mathbb{E} \int_{0}^{t} \int_{0}^{\infty}\left\{\sigma_{s}^{2} \tilde{\rho}_{s}^{2}-\sigma_{s}^{2}+2 \theta\right\}\left|\mathcal{T}_{\varepsilon} \Delta_{s}\right|^{2} d x d s+o(1), \quad
\end{aligned}
$$

as $\varepsilon \downarrow 0$, for a constant $C_{\theta}$ which only depends on the free parameter $\theta$.

Step 5. Since $\rho$ is bounded away from 1 and $\sigma$ is bounded away from 0 , we can take the free parameter $\theta$ sufficiently small so that (for all $x$ and $t$ )

$$
\sigma(s, x)^{2} \rho\left(t, \tilde{\nu}_{t}\right)^{2}-\sigma(s, x)^{2}+2 \theta \leq-c_{0}
$$

for a fixed constant $c_{0}>0$. Next, we can consider the stopping times

$$
t_{n}:=\inf \left\{t>0:\|\mathcal{T} \nu\|_{t, \star}^{2}>n \text { or }|M|_{t, \star}^{2}>n\right\} \wedge T,
$$

for $n \geq 1$, and notice that, by Proposition 5.3 , we have $t_{n} \uparrow T$ as $n \rightarrow \infty$. Evaluating the estimate (5.15) at $t \wedge t_{n}$ and using (5.16), we get

$$
\begin{aligned}
& \mathbb{E}\left\|\partial_{x}^{-1} \mathcal{T}_{\varepsilon} \Delta_{t \wedge t_{n}}\right\|_{2}^{2}+c_{0} \mathbb{E} \int_{0}^{t \wedge t_{n}}\left\|\mathcal{T}_{\varepsilon} \Delta_{s}\right\|_{2}^{2} d s \leq C n \int_{0}^{t} \mathbb{E}\left\|\partial_{x}^{-1} \mathcal{T}_{\varepsilon} \Delta_{s \wedge t_{n}}\right\|_{2}^{2} d s+o(1) \\
& +C n^{2} \mathbb{E} \int_{0}^{t \wedge t_{n}}\left|L_{s}-\tilde{L}_{s}\right|^{2}+d_{1}\left(\nu_{s}, \tilde{\nu}_{s}\right)^{2} d s+C n^{2} \mathbb{E} \int_{0}^{t \wedge t_{n}} d_{0}\left(\nu_{s}, \tilde{\nu}_{s}\right)\left\|\partial_{x}^{-1} \mathcal{T}_{\varepsilon} \Delta_{s}\right\|_{2} d s .
\end{aligned}
$$

Finally, by applying the integrating factor $\exp \{-C n t\}$ to the first term on the right-hand side, we obtain the estimate from Proposition 5.1 with $c_{n}:=n^{2}\left(e^{C T}-1\right)$.

\section{Density Estimates}

The purpose of this section is to prove the density estimates stated in Proposition 3.3. Our approach will rely on techniques that are entirely probabilistic in nature and we follow a simple intuitive procedure.

The first step is to ensure sufficiently fast decay of the tails of the particles. This is achieved in Section 6.1, where we show that the sub-Gaussianity of the initial law propagates nicely for all positive times. In Section 6.2, we transform the particles into Brownian motions with drift that have the same hitting times of the origin, and then we use the sub-Gaussianity to introduce a change of measure related to the drift.

Finally, we derive the density estimates in Section 6.3, by comparing any given transformed particle at time $t>0$ with an independent absorbed Brownian motion (under the original measure) started from the transformed particle's position at earlier times $s<t$ and run for the remaining time $t-s$. 


\subsection{Sub-Gaussianity}

For notational convenience, we define the dominating processes

$$
\Lambda_{t}^{i, N}:=\left|X_{t}^{i}\right|+\sum_{j=1}^{N} a_{j}^{N}\left|X_{t}^{j}\right|, \quad \Gamma_{t}^{i, N}:=\left|X_{t}^{i}\right|^{2}+\sum_{j=1}^{N} a_{j}^{N}\left|X_{t}^{j}\right|^{2} .
$$

Notice that, by Assumption 2.1, we have

$$
\left|X_{t}^{i}\right|,\left|M_{t}^{N}\right|,\left|b\left(t, X_{t}^{i}, \nu_{t}^{N}\right)\right| \lesssim 1+\Lambda_{t}^{i, N} .
$$

Proposition 6.1. Let Assumption 2.1 be satisfied. For every $\epsilon>0$, there is a (smooth) decreasing function $t \mapsto \eta_{t}$ with $\eta_{0}=\epsilon / 2$ and $\eta_{t}>0$ such that, for all $t>0$ and $N \geq 1$,

$$
\mathbb{E} e^{\eta_{t} \Gamma_{t}^{i, N}} \leq e^{c \int_{0}^{t} \eta_{s} d s} \mathbb{E}\left[e^{\epsilon\left|X_{0}^{i}\right|^{2}}\right]^{\frac{1}{2}} \mathbb{E}\left[e^{\epsilon \sum_{j=1}^{N} a_{j}^{N}\left|X_{0}^{j}\right|^{2}}\right]^{\frac{1}{2}} .
$$

In particular, $X_{t}^{i}, M_{t}^{N}$, and $\Lambda_{t}^{i, N}$ are all sub-Gaussian uniformly in $N \geq 1$.

Proof. First of all, the only fact we will use about the drift is $\left|b_{t}^{i}\right| \lesssim 1+\Lambda_{t}^{i, N}$, so noting that $\left|b_{t}^{i}-\left(\mathfrak{L}^{N}\right)_{t}^{\prime} \alpha_{t}^{i}\right| \lesssim 1+\Lambda_{t}^{i, N}$, we can assume without loss of generality that $\alpha_{t}^{i} \equiv 0$.

Fix a strictly positive (deterministic) function $\eta \in \mathcal{C}^{1}\left(\mathbb{R}_{+}\right)$with $\eta_{0}=\epsilon / 2$ and define $\Psi \in \mathcal{C}^{1,2}\left(\mathbb{R}_{+} \times \mathbb{R}^{N}\right)$ by

$$
\Psi(t, x):=e^{\eta_{t}\left(x_{i}^{2}+\sum_{j} a_{j}^{N} x_{j}^{2}\right)} .
$$

For simplicity of notation, we write $\Gamma_{t}$ in place of $\Gamma_{t}^{i, N}$. Then $\Psi\left(t, X_{t}\right)=e^{\eta_{t} \Gamma_{t}}$, where we have defined $X_{t}:=\left(X_{t}^{1}, \ldots, X_{t}^{N}\right)$. Introducing the stopping times

$$
\tau_{n}:=\inf \left\{t \geq 0:\left|X_{t}\right|>n\right\} \quad \text { for } n \geq 1,
$$

and applying Itô's formula, we get

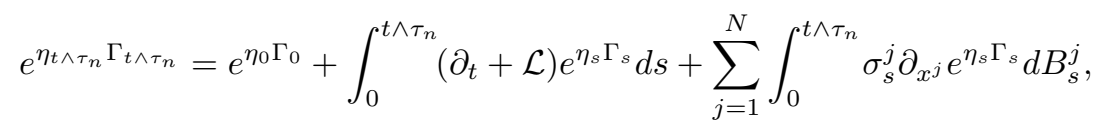

where $d B_{s}^{j}=\rho_{t} d W_{t}^{0}+\sqrt{1-\rho_{t}^{2}} d W_{t}^{j}$ and

$$
\mathcal{L} \Psi=\sum_{j=1}^{N} b_{t}^{j} \partial_{x_{j}} \Psi+\frac{1}{2} \sum_{j=1}^{N}\left(\sigma_{t}^{j}\right)^{2} \partial_{x_{j}}^{2} \Psi+\frac{1}{2} \sum_{k \neq j} \sigma_{t}^{j} \sigma_{t}^{k} \rho_{t}^{2} \partial_{x_{j} x_{k}} \Psi .
$$

Computing the derivatives, we see that

$$
\begin{aligned}
\left(\partial_{t}+\mathcal{L}\right) e^{\eta_{t} \Gamma_{t}}=e^{\eta_{t} \Gamma_{t}} & \left(\dot{\eta}_{t} \Gamma_{t}+2 \eta_{t} \sum_{j=1}^{N} b_{t}^{j}\left(\delta_{i j}+a_{j}^{N}\right) X_{t}^{j}+\right. \\
& +\eta_{t} \sum_{j=1}^{N}\left(\sigma_{t}^{j}\right)^{2}\left(\delta_{i j}+a_{j}\right)+2 \eta_{t}^{2} \sum_{j=1}^{N}\left(\sigma_{t}^{j}\right)^{2}\left(\delta_{i j}+a_{j}^{N}\right)^{2}\left(X_{t}^{j}\right)^{2} \\
& \left.+2 \eta_{t}^{2} \rho_{t}^{2} \sum_{k \neq j} \sigma_{t}^{j} \sigma_{t}^{k}\left(\delta_{i j}+a_{j}^{N}\right)\left(\delta_{i k}+a_{k}^{N}\right) X_{t}^{j} X_{t}^{k}\right) .
\end{aligned}
$$


Using the bounds $\left|\sigma^{j}\right| \leq C_{\sigma}$ and $\left|b^{j}\right| \leq C_{b}\left(1+\left|X_{t}^{j}\right|+\sum_{\ell} a_{\ell}^{N}\left|X_{t}^{\ell}\right|\right)$ as well as the basic inequalities

$$
x \leq 1+x^{2}, \quad(x+y)^{2} \leq 2\left(x^{2}+y^{2}\right), \quad \text { and } \quad\left(\sum_{j=1}^{N} a_{j}^{N} x_{j}\right)^{2} \leq \sum_{j=1}^{N} a_{j}^{N} x_{j}^{2},
$$

one then easily verifies that

$$
\left(\partial_{t}+\mathcal{L}\right) e^{\eta_{t} \Gamma_{t}} \leq\left(2 \eta_{t}\left(C_{b}+C_{\sigma}^{2}\right)+\left(\dot{\eta}_{t}+10 C_{b} \eta_{t}+8 C_{\sigma}^{2} \eta_{t}^{2}\right) \Gamma_{t}\right) e^{\eta_{t} \Gamma_{t}}
$$

Consequently, if only we can choose $\eta \in \mathcal{C}^{1}\left(\mathbb{R}_{+}\right)$such that

$$
\dot{\eta}_{t}+10 C_{b} \eta_{t}+8 C_{\sigma}^{2} \eta_{t}^{2}=0, \quad \eta_{0}=\epsilon / 2,
$$

then we have

$$
\left(\partial_{t}+\mathcal{L}\right) e^{\eta_{t} \Gamma_{t}} \leq 2 \eta_{t}\left(C_{\sigma}^{2}+C_{b}\right) e^{\eta_{t} \Gamma_{t}} .
$$

This is achieved by taking

$$
\eta_{t}:=\frac{5 C_{b} \epsilon}{10 C_{b} e^{10 C_{b} t}+4 C_{\sigma}^{2} \epsilon\left(e^{10 C_{b} t}-1\right)} .
$$

With this choice for $\eta$, the inequality (6.4) is satisfied and thus (6.3) gives that

$$
e^{\eta_{t \wedge \tau_{n}} \Gamma_{t \wedge \tau_{n}}} \leq e^{\eta_{0} \Gamma_{0}}+2\left(C_{\sigma}^{2}+C_{b}\right) \int_{0}^{t \wedge \tau_{n}} \eta_{s} e^{\eta_{s} \Gamma_{s}} d s+\sum_{j=1}^{N} \int_{0}^{t \wedge \tau_{n}} \sigma_{s}^{j} \partial_{x_{j}} e^{\eta_{s} \Gamma_{s}} d B_{s}^{j} .
$$

Taking expectations and noting that the stochastic integral is a true martingale,

$$
\mathbb{E}\left[e^{\eta_{t \wedge \tau_{n}} \Gamma_{t \wedge \tau_{n}}}\right] \leq \mathbb{E}\left[e^{\eta_{0} \Gamma_{0}}\right]+2\left(C_{\sigma}^{2}+C_{b}\right) \int_{0}^{t} \eta_{s} \mathbb{E}\left[e^{\eta_{s \wedge \tau_{n}} \Gamma_{s \wedge \tau_{n}}}\right] d s .
$$

By Cauchy-Schwarz (recall $\left.\eta_{0}=\epsilon / 2\right)$, we have

$$
\mathbb{E}\left[e^{\eta_{0} \Gamma_{0}}\right] \leq \mathbb{E}\left[e^{\epsilon\left|X_{0}^{i}\right|^{2}}\right]^{\frac{1}{2}} \mathbb{E}\left[e^{\epsilon \sum_{j} a_{j}^{N}\left|X_{0}^{j}\right|^{2}}\right]^{\frac{1}{2}}
$$

and hence it follows from Gronwall's inequality that

$$
\mathbb{E}\left[e^{\eta_{t \wedge \tau_{n}} \Gamma_{t \wedge \tau_{n}}}\right] \leq e^{2\left(C_{\sigma}^{2}+C_{b}\right) \int_{0}^{t} \eta_{s} d s} \mathbb{E}\left[e^{\epsilon\left|X_{0}^{i}\right|^{2}}\right]^{\frac{1}{2}} \mathbb{E}\left[e^{\epsilon \sum_{j} a_{j}^{N}\left|X_{0}^{j}\right|^{2}}\right]^{\frac{1}{2}},
$$

Noting that $\tau_{n} \uparrow \infty$ as $n \rightarrow \infty$, we have $e^{\eta_{t \wedge \tau_{n}} \Gamma_{t \wedge \tau_{n}}} \rightarrow e^{\eta_{t} \Gamma_{t}}$ as $n \rightarrow \infty$, by the continuity of $t \mapsto \eta_{t}$ and $t \mapsto X_{t}^{j}$, for $j=1, \ldots, N$. Consequently, Fatou's lemma yields

$$
\mathbb{E}\left[e^{\eta_{t} \Gamma_{t}}\right] \leq \liminf _{n \rightarrow \infty} \mathbb{E}\left[e^{\eta_{t \wedge \tau_{n}} \Gamma_{t \wedge \tau_{n}}}\right] \leq e^{2\left(C_{\sigma}^{2}+C_{b}\right) \int_{0}^{t} \eta_{s} d s} \mathbb{E}\left[e^{\epsilon\left|X_{0}^{i}\right|^{2}}\right]^{\frac{1}{2}} \mathbb{E}\left[e^{\epsilon \sum_{j} a_{j}^{N}\left|X_{0}^{j}\right|^{2}}\right]^{\frac{1}{2}} .
$$

Since $\eta_{t}$ does not depend on $N$, the estimate is uniform in $N \geq 1$.

The above result also yields control over the running maxima of the processes. 
Corollary 6.2. Given $\eta_{T}$ from Proposition 6.1. fix any $\epsilon<\eta_{T} / 2$. Then we have

$$
\mathbb{E}\left[e^{\epsilon \sup _{t \leq T} \Gamma_{t}^{i, N}}\right] \leq C_{T, \epsilon}
$$

for some constant $C_{T, \epsilon}>0$ that is uniform in $N \geq 1$.

Proof. Set $\xi_{t}:=p \eta_{t} / 2$ for $p<1$ and derive the estimate 6.6 for $\xi$. As $p<1,\left|\partial_{x_{j}} e^{\xi_{s} \Gamma_{s}}\right|^{2}$ is integrable by Proposition 6.1, so we can apply Burkholder-Davis-Gundy to control the running max of the stochastic integral. Thus, taking expectation and using monotone convergence, we get

$$
\mathbb{E}\left[e^{\frac{p \eta_{T}}{2} \sup _{t \leq T} \Gamma_{t}}\right] \leq \mathbb{E}\left[\sup _{t \leq T} e^{\xi_{t} \Gamma_{t}}\right] \leq C_{T, p} .
$$

As $p<1$ was arbitrary, the claim follows.

\subsection{Change of measure}

In this section we first transform each particle into a Brownian motion with drift in a way that preserves the hitting times of the origin. Next, we then use the sub-Gaussianity to introduce a change-of-measure that can remove this drift and finally we obtain an important estimate for the associated Radon-Nikodym derivative.

Lemma 6.3. Define the transformation $\Upsilon \in C^{1,2}([0, T] \times \mathbb{R})$ by

$$
(t, x) \mapsto \Upsilon_{t}(x):=\int_{0}^{x} \frac{1}{\sigma(t, y)} d y .
$$

Fixing an arbitrary index $i \in\{1, \ldots, N\}$, we let $Z_{t}:=\Upsilon_{t}\left(X_{t}^{i}\right)$. Then

$$
d Z_{t}=\hat{b}_{t}^{i} d t+d B_{t}^{i} \quad \text { with } \quad Z_{0}=\Upsilon_{0}\left(X_{0}^{i}\right),
$$

where $B^{i}$ is a Brownian motion and the (stochastic) drift $\hat{b}_{t}^{i}$ obeys the growth condition

$$
\left|\hat{b}_{t}^{i}\right| \lesssim 1+\left|X_{t}^{i}\right|+\left|M_{t}^{N}\right| \text {. }
$$

Furthermore, the transformed process $Z$ satisfies

$$
\operatorname{sgn}\left(Z_{t}\right)=\operatorname{sgn}\left(X_{t}^{i}\right) \quad \text { and } \quad\left|Z_{t}\right| \lesssim\left|X_{t}^{i}\right| .
$$

Proof. Note that

$$
\partial_{t} \Upsilon_{t}(x)=-\int_{0}^{x} \frac{\partial_{t} \sigma(t, y)}{\sigma(t, y)^{2}} d y, \quad \partial_{x} \Upsilon_{t}(x)=\frac{1}{\sigma(t, x)}, \quad \partial_{x x}^{2} \Upsilon_{t}(x)=-\frac{\partial_{x} \sigma(t, x)}{\sigma(t, x)^{2}}
$$

Defining $B_{t}^{i}:=\int_{0}^{t} \rho_{s} d W_{s}^{0}+\int_{0}^{t} \sqrt{1-\rho_{s}^{2}} d W_{s}^{i}$, we have $d X_{t}=\left(b_{t}^{i}-\alpha_{t}^{i}\left(\mathfrak{L}^{N}\right)_{t}^{\prime}\right) d t+\sigma_{t} d B_{t}$ with $d\langle X\rangle_{t}=\sigma_{t}^{2} d t$. Hence an application of Itô's formula yields $d Z_{t}=\hat{b}_{t}^{i} d t+d B_{t}^{i}$, where

$$
\hat{b}_{t}^{i}:=\frac{b_{t}\left(X_{t}^{i}\right)-\alpha_{t}\left(X_{t}^{i}\right)\left(\mathfrak{L}^{N}\right)_{t}^{\prime}}{\sigma\left(t, X_{t}^{i}\right)}-\frac{1}{2} \partial_{x} \sigma\left(t, X_{t}^{i}\right)-\int_{0}^{X_{t}^{i}} \frac{\partial_{t} \sigma(t, y)}{\sigma(t, y)^{2}} d y .
$$

Now, the bound on $Z_{t}$ and the statement about its sign in 6.9 follow directly from the definition of $\Upsilon$, since $\sigma$ is strictly positive and bounded away from zero. Similarly, the growth condition in 6.8 follows from the properties of the coefficients (Assumption 2.1) and the fact that $\left|\left(\mathfrak{L}^{N}\right)_{t}^{\prime}\right| \leq \int_{0}^{t}\left|\mathfrak{K}^{\prime}(t-s)\right| L_{s}^{N} d s \leq\left\|\mathfrak{K}^{\prime}\right\|_{L^{1}}$. 
Lemma 6.4. Fix $i \in\{1, \ldots, N\}$ and define the stochastic exponential

$$
\mathcal{E}_{t}:=\exp \left\{-\int_{0}^{t} \hat{b}_{s}^{i} d B_{s}^{i}-\frac{1}{2} \int_{0}^{t}\left(\hat{b}_{s}^{i}\right)^{2} d s\right\}
$$

where $\hat{b}^{i}$ is the drift of $Z$ as defined in Lemma 6.3. Then $Z$ is a Brownian motion under the probability measure $\mathbb{Q}$ given by the Radon-Nikodym derivative

$$
\left.\frac{d \mathbb{Q}}{d \mathbb{P}}\right|_{\mathcal{F}_{t}}=\mathcal{E}_{t}
$$

with initial value $Z_{0}$ distributed according to $\varpi_{0}:=\mu_{0} \circ \Upsilon_{0}^{-1}$.

Proof. For notational convenience we drop the superscript $i$ and define

$$
\mathcal{E}_{s, t}:=\exp \left\{-\int_{s}^{t} \hat{b}_{u} d B_{u}-\frac{1}{2} \int_{s}^{t} \hat{b}_{u}^{2} d u\right\}, \quad \mathcal{E}_{0, t}=\mathcal{E}_{t} .
$$

By standard arguments, $\mathcal{E}_{t}$ is a positive continuous local martingale and hence also a supermartingale with $\mathcal{E}_{0}=1$. The claim is now that $\mathcal{E}_{t}$ is in fact a true martingale on $[0, T]$, which amounts to showing that $\mathbb{E} \mathcal{E}_{t}=1$ for all $t \in[0, T]$.

Recall that $\left|\hat{b}_{s}\right| \leq C\left(1+\Lambda_{s}^{i, N}\right)$. While we cannot appeal to Novikov's condition directly, the sub-Gaussianity of $\Lambda_{s}^{i, N}$ will allows us to apply it on every interval of a fine enough partition of $[0, T]$. To see this, we fix an arbitrary $n \geq 1$ and partition the interval $[0, T]$ by $t_{0}<\cdots<t_{n}$ where $t_{k}:=k T / n$. An application of Jensen's inequality then yields

$$
\begin{aligned}
\mathbb{E} \exp \left\{\frac{1}{2} \int_{t_{k-1}}^{t_{k}} \hat{b}_{u}^{2} d u\right\} & \leq \frac{n}{T} \mathbb{E} \int_{t_{k-1}}^{t_{k}} \exp \left\{\frac{T}{2 n} \hat{b}_{u}^{2}\right\} d u=\frac{n}{T} \int_{t_{k-1}}^{t_{k}} \mathbb{E} \exp \left\{\frac{T}{2 n} \hat{b}_{u}^{2}\right\} d u \\
& \leq \sup _{t \in\left[t_{k-1}, t_{k}\right]} \mathbb{E} \exp \left\{\frac{C^{2} T}{n}\left(1+\Lambda_{t}^{i, N}\right)^{2}\right\} .
\end{aligned}
$$

Choosing $n \geq 1$ sufficiently large so that $2 C^{2} T / n \leq \eta_{T}$, we deduce from Proposition 6.1 that

$$
\mathbb{E} \exp \left\{\frac{1}{2} \int_{t_{k-1}}^{t_{k}} \hat{b}_{u}^{2} d u\right\}<\infty, \quad \text { for each } k=1, \ldots, n .
$$

In particular, Novikov's condition now implies that $\left(\mathcal{E}_{t}\right)_{t \in\left[0, t_{1}\right]}$ is a true martingale and hence

$$
\mathbb{E} \mathcal{E}_{t}=1, \quad \text { for all } t \in\left[0, t_{1}\right]
$$

Noting that $\left(\mathcal{E}_{t_{1}, t}\right)_{t \in\left[t_{1}, t_{2}\right]}$ is again a stochastic exponential with $\mathcal{E}_{t_{1}, t_{1}}=1$, another application of Novikov's condition shows that $\left(\mathcal{E}_{t_{1}, t}\right)_{t \in\left[t_{1}, t_{2}\right]}$ is a martingale. Consequently, we have

and hence

$$
\mathbb{E}\left[\mathcal{E}_{t_{1}, t} \mid \mathcal{F}_{t_{1}}\right]=\mathcal{E}_{t_{1}, t_{1}}=1
$$

$$
\mathbb{E} \mathcal{E}_{t}=\mathbb{E}\left[\mathcal{E}_{t_{1}} \mathcal{E}_{t_{1}, t}\right]=\mathbb{E}\left[\mathcal{E}_{t_{1}} \mathbb{E}\left[\mathcal{E}_{t_{1}, t} \mid \mathcal{F}_{t_{1}}\right]\right]=\mathbb{E}\left[\mathcal{E}_{t_{1}}\right]=1
$$

for all $t \in\left[t_{1}, t_{2}\right]$. Considering inductively the intervals $\left[t_{k-1}, t_{k}\right]$ for $k=3, \ldots, n$, it follows by the same reasoning that $\mathbb{E} \mathcal{E}_{t}=1$ for all $t \in[0, T]$. Thus; $\mathcal{E}_{t}$ is a true martingale on $[0, T]$ and hence Girsanov's theorem implies that the process

$$
d Z_{t}=\hat{b}_{t} d t+d B_{t}, \quad Z_{0}=\Upsilon_{0}\left(X_{0}^{1}\right),
$$

is a Brownian motion under $\mathbb{Q}$ with initial distribution $\varpi_{0}=\mu_{0} \circ \Upsilon_{0}^{-1}$. 
Other than $Z$ being a Brownian motion under $\mathbb{Q}$, we shall also need a specific estimate on the Radon-Nikodym derivative, $\mathcal{E}_{t}$, from the previous lemma.

Lemma 6.5 (Radon-Nikodym estimate). Let $\mathcal{E}_{t}$ be the stochastic exponential defined in Lemma 6.4. Given any $\epsilon>0$ such that $\mathbb{E} \exp \left\{\epsilon\left|X_{0}^{j}\right|^{2}\right\}<\infty$, for $j=1, \ldots, N$, there exists $p>1$ close enough to 1 such that

$$
\mathbb{E}\left[\mathcal{E}_{t}^{1-p} \mid X_{0}^{i}=x_{0}\right] \leq C \exp \left\{\epsilon x_{0}^{2}\right\}, \quad \text { where } \quad C=C(p, \epsilon, T)
$$

Moreover, for any $q>1$, we have

$$
\mathbb{E}\left[\left|\hat{b}_{t}^{i}\right|^{q} \mid X_{0}^{i}=x_{0}\right] \leq C\left(1+x_{0}^{q}\right), \quad \text { where } \quad C=C(q, T) .
$$

Proof. We begin by defining

$$
Y_{t}:=-\int_{0}^{t} \hat{b}_{s}^{i} d B_{s}^{i}
$$

Using Hölder's inequality with $p>1$, we then have

$$
\begin{aligned}
\mathbb{E}\left[\mathcal{E}_{t}^{1-p} \mid X_{0}^{i}=x_{0}\right] & =\mathbb{E}\left[\left(e^{p Y_{t}-\frac{1}{2}\langle p Y\rangle_{t}}\right)^{\frac{p-1}{p}}\left(e^{\frac{(p+1)}{2}(p-1) p\langle Y\rangle_{t}}\right)^{\frac{1}{p}} \mid X_{0}^{i}=x_{0}\right] \\
& \leq \mathbb{E}\left[e^{p Y_{t}-\frac{1}{2}\langle p Y\rangle_{t}} \mid X_{0}^{i}=x_{0}\right]^{\frac{p-1}{p}} \mathbb{E}\left[e^{\frac{(p+1)}{2}(p-1) p\langle Y\rangle_{t}} \mid X_{0}^{i}=x_{0}\right]^{\frac{1}{p}}
\end{aligned}
$$

Noting that the first term on the right-hand side is bounded by 1 , we conclude that

$$
\mathbb{E}\left[\mathcal{E}_{t}^{1-p} \mid X_{0}^{i}=x_{0}\right] \leq \mathbb{E}\left[e^{C_{p} \int_{0}^{t}\left(\hat{b}_{s}^{i}\right)^{2} d s} \mid X_{0}^{i}=x_{0}\right]^{\frac{1}{p}} \quad \text { with } \quad C_{p}:=\frac{(p+1)}{2}(p-1) p .
$$

The crucial observation here is that $C_{p} \downarrow 0$ as $p \downarrow 1$. Recalling the bound $\left|\hat{b}_{s}^{i}\right| \lesssim 1+\Lambda_{s}^{i, N}$, we can apply Jensen's inequality to see that

$$
\mathbb{E}\left[\mathcal{E}_{t}^{1-p} \mid X_{0}^{i}=x_{0}\right] \lesssim\left(\frac{1}{T} \int_{0}^{T} \mathbb{E}\left[e^{T C C_{p}\left(\Lambda_{s}^{i, N}\right)^{2}} \mid X_{0}^{i}=x_{0}\right] d s\right)^{\frac{1}{p}}
$$

Fix a power $p_{0}$ close enough to 1 such that $T C C_{p_{0}} \leq \eta_{T} / 2$, with $\eta$ as in Proposition 6.1. so that

$$
\mathbb{E}\left[\mathcal{E}_{t}^{1-p_{0}} \mid X_{0}^{i}=x_{0}\right] \lesssim \sup _{s \in[0, T]} \mathbb{E}\left[e^{\eta_{s}\left(\Lambda_{s}^{i, N}\right)^{2} / 2} \mid X_{0}^{i}=x_{0}\right]^{\frac{1}{p_{0}}}
$$

Now, recalling the form of the estimate 6.7 in the proof of Proposition 6.1 the fact that $X_{0}^{i}$ is independent of $X_{0}^{j}$ for $j \neq i$ implies that

$$
\sup _{s \in[0, T]} \mathbb{E}\left[e^{\eta_{T}\left(\Lambda_{s}^{i, N}\right)^{2} / 2} \mid X_{0}^{i}=x_{0}\right] \leq C e^{\epsilon x_{0}^{2} / p_{0}} \mathbb{E}\left[e^{\epsilon \sum_{j \neq i} a_{j}^{N}\left(X_{0}^{j}\right)^{2}}\right]^{\frac{1}{2 p_{0}}}
$$

Combining this with the estimate 6.10 , the first claim of the proposition follows.

Finally, using the bound $\left|\hat{b}_{t}^{i}\right| \lesssim 1+\Lambda_{t}^{\imath, N}$, the second claim follows by a standard Gronwall argument, so we leave out the proof. 


\subsection{The density estimates}

The purpose of this section is to derive the desired density estimates for $X_{t}^{i}$ by controlling the probability $\mathbb{P}\left(X_{t}^{i} \in S, t<\tau_{i}\right)$, for any given $i \in\{1, \ldots, N\}$ and $S \in \mathcal{B}(0, \infty)$.

Recall the transformation $\Upsilon$ from Lemma 6.3 and note that $x \mapsto \Upsilon_{t}(x)$ is bijective with $\Upsilon_{t}(x) \leq 0$ if and only if $x \leq 0$. Hence, conditioning on the initial value $X_{0}^{i}=x_{0}$,

$$
\mathbb{P}^{x_{0}}\left(X_{t}^{i} \in S, t<\tau_{i}\right)=\mathbb{P}^{z}\left(Z_{t} \in S_{t}, t<\tau\right),
$$

where $\tau=\inf \left\{t>0: Z_{t} \leq 0\right\}, Z_{t}=\Upsilon\left(X_{t}^{i}\right), S_{t}=\Upsilon_{t}(S)$, and $z=\Upsilon_{0}\left(x_{0}\right)$.

From here, the idea is to approximate the transformed particle $Z_{t \wedge \tau}$ by running it up to time $s$, for $s<t$, and then running an independent absorbed Brownian motion $W$ for the remaining time $t-s$. More precisely, given $z \in(0, \infty)$, we are interested in the map

$$
s \mapsto \mathbb{E}^{z}\left[\mathbb{P}^{Z_{s \wedge \tau}}\left(W_{t-s} \in S_{t}, t-s<\tau_{W}\right)\right],
$$

where $\tau_{W}=\inf \left\{t>0: W_{t} \leq 0\right\}$. For a fixed time $t \in[0, T]$, we therefore define, for every $s<t$, the function

$$
u(s, x):=\mathbb{P}^{x}\left(W_{t-s} \in S_{t}, t-s<\tau_{W}\right)=\int_{S_{t}} G_{t-s}(y, x) d y,
$$

where $G_{t}(y, x)=p_{t}(x-y)-p_{t}(x+y)$ with $p_{t}(x)=(2 \pi t)^{-\frac{1}{2}} \exp \left\{-x^{2} / 2 t\right\}$. Note that $u$ is a classical solution of the terminal-boundary value problem

$$
\left\{\begin{array}{lll}
\partial_{s} u(s, x)+\frac{1}{2} \Delta u(s, x)=0 & \text { on } & {[0, t) \times(0, \infty)} \\
u(t, x)=\mathbf{1}_{S_{t}}(x) & \text { on } & \{t\} \times(0, \infty) \\
u(s, 0)=0 & \text { on } & {[0, t) \times\{0\}}
\end{array}\right.
$$

We can write (6.11) more succinctly as

$$
s \mapsto v(s, z):=\mathbb{E}^{z}\left[u\left(s, Z_{s \wedge \tau}\right)\right],
$$

and note that

$$
v(0, z)=\mathbb{P}^{z}\left(W_{t \wedge \tau_{W}} \in S_{t}\right) \quad \text { and } \quad v(t, z)=\mathbb{P}^{z}\left(Z_{t \wedge \tau} \in S_{t}\right) .
$$

By bounded convergence, it is immediate that $s \mapsto v(s, z)$ is continuous. Additionally, we show in Lemma 6.6 below that it is in fact absolutely continuous on $\left[0, t_{0}\right]$ for any $t_{0}<t$. Consequently, if only we can show that the (a.e.) derivative $\partial_{s} v$ extends to $L^{1}(0, t)$, then we will have absolute continuity on all of $[0, t]$ with

$$
\mathbb{P}^{z}\left(Z_{t \wedge \tau} \in S_{t}\right)=\mathbb{P}^{z}\left(W_{t \wedge \tau_{W}} \in S_{t}\right)+\int_{0}^{t} \partial_{s} v(s, z) d s .
$$

Therefore, the key is simply to establish the right control over $s \mapsto \partial_{s} v(s, z)$. We embark on this in the next section, but first we prove the previous claim about the absolute continuity.

Lemma 6.6. Fix an arbitrary $z \in(0, \infty)$. Then the mapping $s \mapsto v(s, z)$ from (6.13) is in $A C\left[0, t_{0}\right]$ for every $t_{0}<t$ with (a.e.) derivative

$$
\partial_{s} v(s, z)=\mathbb{E}^{z}\left[\mathbf{1}_{s<\tau} \hat{b}_{s} \partial_{x} u\left(s, Z_{s}\right)\right] .
$$


Proof. Fix $s \in[0, t)$ and $h>0$ such that $|h|<t-s$. Recalling Lemma 6.3, we have $d Z_{t \wedge \tau}=\mathbf{1}_{t<\tau} \hat{b}_{t} d t+\mathbf{1}_{t<\tau} d B_{t}$, so an application of Itô's formula yields

$$
\begin{aligned}
v(s+h, z) & -v(s, z)=\mathbb{E}^{z}\left[u\left(s+h, Z_{(s+h) \wedge \tau}\right)-u\left(s, Z_{s \wedge \tau}\right)\right] \\
& =\mathbb{E}^{z}\left[\int_{s}^{s+h}\left(\partial_{s}+\frac{1}{2} \triangle\right) u_{r} d r+\int_{s}^{s+h} \mathbf{1}_{r<\tau} \hat{b}_{r} \partial_{x} u_{r} d r+\int_{s}^{s+h} \mathbf{1}_{r<\tau} \partial_{x} u_{r} d B_{r}\right] .
\end{aligned}
$$

The first term on the right-hand side vanishes by 6.12 and, since $\partial_{x} u(r, x)$ is bounded on the interval $[s, s+h] \subseteq[0, t)$, the stochastic integral is a true martingale. Noting also that $\mathbb{E}\left|\sup _{r \leq T} \hat{b}_{r}\right|<\infty$ (see e.g. Corollary 6.2), Fubini's theorem thus implies that

$$
v(s+h, z)-v(s, z)=\int_{s}^{s+h} \mathbb{E}^{z}\left[\mathbf{1}_{r<\tau} \hat{b}_{r} \partial_{x} u\left(r, Z_{r}\right)\right] d r .
$$

This proves the claim.

\subsubsection{Estimates on the half-line}

Given the expression for $\partial_{s} v(s, z)$ in Lemma 6.6, an application of Hölder's inequality with $q>1$ yields

$$
\begin{aligned}
\left|\partial_{s} v(s, z)\right| & \leq \mathbb{E}^{z}\left[\mathbf{1}_{s<\tau}\left|\hat{b}_{s}\right|\left|\partial_{x} u\left(s, Z_{s}\right)\right|\right]=\mathbb{E}^{z}\left[\mathbf{1}_{s<\tau}\left|\hat{b}_{s}\right| \int_{S_{t}}\left|\partial_{x} G_{t-s}\left(y, Z_{s}\right)\right| d y\right] . \\
& \leq \mathbb{E}\left[\left|\hat{b}_{s}\right|^{\frac{q}{q-1}} \mid X_{0}^{i}=x_{0}\right]^{\frac{q-1}{q}} \int_{S_{t}} \mathbb{E}^{z}\left[\mathbf{1}_{s<\tau}\left|\partial_{x} G_{t-s}\left(y, Z_{s}\right)\right|^{q}\right]^{\frac{1}{q}} d y \\
& \leq C\left(1+x_{0}\right) \int_{S_{t}} \mathbb{E}^{z}\left[\mathbf{1}_{s<\tau}\left|\partial_{x} G_{t-s}\left(y, Z_{s}\right)\right|^{q}\right]^{\frac{1}{q}} d y .
\end{aligned}
$$

Here the last inequality follows from the second claim in Lemma 6.5 and we emphasize that $q$ can be taken arbitrarily close to 1 . By introducing the Radon-Nikodym derivative $\mathcal{E}$ from Lemma 6.4. Hölder's inequality with $p>1$ yields

$$
\begin{aligned}
\int_{S_{t}} \mathbb{E}^{z}\left[\mathbf{1}_{s<\tau}\left|\partial_{x} G_{t-s}\left(y, Z_{s}\right)\right|^{q}\right]^{\frac{1}{q}} d y=\int_{S_{t}} \mathbb{E}_{\mathbb{Q}}^{z}\left[\mathcal{E}_{s}^{-1} \mathbf{1}_{s<\tau}\left|\partial_{x} G_{t-s}\left(y, Z_{s}\right)\right|^{q}\right]^{\frac{1}{q}} d y \\
\leq \mathbb{E}\left[\mathcal{E}_{s}^{1-p} \mid X_{0}^{i}=x_{0}\right]^{\frac{1}{p q}} \int_{S_{t}} \mathbb{E}_{\mathbb{Q}}^{z}\left[\mathbf{1}_{s<\tau}\left|\partial_{x} G_{t-s}\left(y, Z_{s}\right)\right|^{a}\right]^{\frac{1}{a}} d y
\end{aligned}
$$

where $a=a(p, q):=q \frac{p}{p-1}>1$. For any $\delta>0$, we can take $p$ close enough to 1 so that the first estimate from Lemma 6.5 applies. Consequently, there exists $a>1$ large enough such that

$$
\begin{aligned}
\left|\partial_{s} v(s, z)\right| & \lesssim e^{\delta x_{0}^{2}} \int_{S_{t}} \mathbb{E}_{\mathbb{Q}}^{z}\left[\mathbf{1}_{s<\tau}\left|\partial_{x} G_{t-s}\left(y, Z_{s \wedge \tau}\right)\right|^{a}\right]^{\frac{1}{a}} d y \\
& =e^{\delta x_{0}^{2}} \int_{S_{t}}\left(\int_{0}^{\infty}\left|\partial_{x} G_{t-s}(y, x)\right|^{a} G_{s}(x, z) d x\right)^{\frac{1}{a}} d y,
\end{aligned}
$$

where the last line follows from the fact that $Z_{s \wedge \tau}$ is an absorbing Brownian motion un$\operatorname{der} \mathbb{Q}$, as shown in Lemma 6.4. Before proceeding, we collect some useful bounds for exponential functions. 
Lemma 6.7. Fix any $x, y \in \mathbb{R}$ and $t>s$. Then it holds for all powers $a \geq 1$ that

$$
\begin{aligned}
& e^{-\frac{a(y-x)^{2}}{2(t-s)}} e^{-\frac{(x-z)^{2}}{2 s}} \leq e^{-\frac{(y-z)^{2}}{2 t}} e^{-\frac{t}{2 s}\left(\frac{(x-y)}{\sqrt{t-s}}+\frac{\sqrt{t-s}(y-z)}{t-s+s a}\right)^{2}}, \\
& e^{-\frac{a(y+x)^{2}}{2(t-s)}} e^{-\frac{(x-z)^{2}}{2 s}} \leq e^{-\frac{(y+z)^{2}}{2 t}} e^{-\frac{t}{2 s}\left(\frac{(y+x)}{\sqrt{t-s}}+\frac{\sqrt{t-s}(y+z)}{t-s+s a}\right)^{2}} .
\end{aligned}
$$

Recalling (6.14), the desired density estimates will follow if we can obtain suitable bounds on the right-hand side of 6.15. Our first result is the following.

Proposition 6.8. For any $\delta>0$ there exists a $>1$ such that, for every $S \in \mathcal{B}((0, \infty))$, it holds uniformly in $N \geq 1$ that

$$
\begin{aligned}
\mathbb{P}^{x_{0}}\left(X_{t \wedge \tau_{i}}^{i, N} \in S\right) & \leq \int_{S} G_{t}\left(\Upsilon_{t}(x), \Upsilon_{0}\left(x_{0}\right)\right) \partial_{x} \Upsilon_{t}(x) d x \\
& +C_{a} e^{\delta x_{0}^{2}} \int_{S}\left(t^{-\frac{1}{a}} \Upsilon_{0}\left(x_{0}\right)^{\frac{1}{a}} \Upsilon_{t}(x)^{\frac{1}{a}} \wedge 1\right) e^{-\frac{\left(\Upsilon_{t}(x)-\Upsilon_{0}\left(x_{0}\right)\right)^{2}}{4 a t}} \partial_{x} \Upsilon_{t}(x) d x
\end{aligned}
$$

Proof. Given $\delta>0$, we can choose $a>1$ such that 6.15 holds. Writing out the expressions for $\partial_{x} G_{t-s}$ and $G_{s}$, and noting that

$$
e^{-(x-z)^{2} / 2 s}-e^{-(x+z)^{2} / 2 s} \leq\left(\frac{2 x z}{s} \wedge 1\right) e^{-(x-z)^{2} / 2 s},
$$

we get $\left|\partial_{s} v(s, z)\right| \lesssim e^{\delta x_{0}^{2}} I(s)$, where

$$
I(s):=s^{-\frac{1}{2 a}}(t-s)^{-\frac{1}{2}} \int_{S_{t}}\left(\int_{0}^{\infty}\left|\frac{(y-x)}{t-s} e^{-\frac{(y-x)^{2}}{2(t-s)}}+\frac{(y+x)}{t-s} e^{-\frac{(y+x)^{2}}{2(t-s)}}\right|^{a} \frac{x z}{s} e^{-\frac{(x-z)^{2}}{2 s}} d x\right)^{\frac{1}{a}} d y .
$$

Recalling 6.14, we thus have

$$
\mathbb{P}^{x}\left(X_{t \wedge \tau_{i}}^{i, N} \in S\right) \leq \mathbb{P}^{z}\left(W_{t} \in S_{t}, t<\tau_{W}\right)+C e^{\delta x^{2}} \int_{0}^{t} I(s) d s
$$

for some $C>0$, so the claim amounts to controlling the integrand $I(s)$. We split the work involved in this endeavour into six steps.

Step 1. To estimate $I(s)$, we begin by observing that

$$
\left|\frac{(y-x)}{t-s} e^{-\frac{(y-x)^{2}}{2(t-s)}}+\frac{(y+x)}{t-s} e^{-\frac{(y+x)^{2}}{2(t-s)}}\right| \leq \frac{|y-x|}{t-s}\left(e^{-\frac{(y-x)^{2}}{2(t-s)}}-e^{-\frac{(y+x)^{2}}{2(t-s)}}\right)+\frac{2 y}{t-s} e^{-\frac{(y+x)^{2}}{2(t-s)}} .
$$

For the first term on the right-hand side, we can use 6.18 to see that

$$
\frac{|y-x|}{t-s}\left(e^{-\frac{(y-x)^{2}}{2(t-s)}}-e^{-\frac{(y+x)^{2}}{2(t-s)}}\right) \leq \frac{|y-x|}{t-s}\left(\frac{2 x y}{t-s} \wedge 1\right) e^{-\frac{(y-x)^{2}}{2(t-s)}}=: f_{1}(s) .
$$

For the second term, it will prove useful to observe that, for $x, y>0$,

$$
\frac{y}{t-s} e^{-\frac{(y+x)^{2}}{2(t-s)}} \leq\left(\frac{x+y}{x}\right)^{\frac{1}{a}} \frac{y}{t-s} e^{-\frac{(y+x)^{2}}{2(t-s)}} \leq y^{\frac{1}{a}} x^{-\frac{1}{a} \frac{(x+y)}{t-s}} e^{-\frac{(y+x)^{2}}{2(t-s)}}=: f_{2}(s),
$$

where we have used that $y^{1-\frac{1}{a}} \leq(x+y)^{1-\frac{1}{a}}$ since $a>1$. 
Based on 6.20 and 6.21, we have $I(s) \leq I_{1}(s)+2 I_{2}(s)$, where

$$
I_{k}(s):=s^{-\frac{3}{2 a}}(t-s)^{-\frac{1}{2}} z^{\frac{1}{a}} \int_{S_{t}}\left(\int_{0}^{\infty} f_{i}(s)^{a} x e^{-\frac{(x-z)^{2}}{2 s}} d x\right)^{\frac{1}{a}} d y, \quad k=1,2 .
$$

Step 2. We begin with the second term, $I_{2}$. Using 6.17) of Lemma 6.7, we have

$$
\begin{aligned}
I_{2}(s) & =s^{-\frac{3}{2 a}} \frac{1}{t-s} \int_{S_{t}} z^{\frac{1}{a}} y^{\frac{1}{a}}\left(\int_{0}^{\infty}\left(\frac{x+y}{\sqrt{t-s}}\right)^{a} e^{-\frac{a(y+x)^{2}}{2(t-s)}} e^{-\frac{(x-z)^{2}}{2 s}} d x\right)^{\frac{1}{a}} d y \\
& \leq s^{-\frac{3}{2 a}} \frac{1}{t-s} \int_{S_{t}} z^{\frac{1}{a}} y^{\frac{1}{a}} e^{\frac{-(y+z)^{2}}{2 t}}\left(\int_{0}^{\infty}\left(\frac{x+y}{\sqrt{t-s}}\right)^{a} e^{-\frac{t}{2 s}\left(\frac{(y+x)}{\sqrt{t-s}}+\frac{\sqrt{t-s}(y+z)}{t-s+s a}\right)^{2}} d x\right)^{\frac{1}{a}} d y
\end{aligned}
$$

To evaluate the inner integral, we perform a change of variables with

$$
w=t^{\frac{1}{2}} s^{-\frac{1}{2}}\left(\frac{(x+y)}{\sqrt{t-s}}+\frac{\sqrt{t-s}(y+z)}{t-s+\sigma s}\right), \quad d x=(t-s)^{\frac{1}{2}} t^{-\frac{1}{2}} s^{\frac{1}{2}} d w
$$

where we note that

$$
0 \leq \frac{(x+y)}{\sqrt{t-s}}=t^{-\frac{1}{2}} s^{\frac{1}{2}} w-\frac{\sqrt{t-s}(y+z)}{t-s+\sigma s} \leq w
$$

and hence

$$
I_{2}(s) \leq s^{-\frac{1}{a}}(t-s)^{\frac{1}{2 a}-1} t^{-\frac{1}{2 a}}\left(\int_{0}^{\infty} w^{a} e^{-\frac{w^{2}}{2}} d w\right)^{\frac{1}{a}} \int_{S_{t}} z^{\frac{1}{a}} y^{\frac{1}{a}} e^{\frac{-(y+z)^{2}}{2 a t}} d y .
$$

Since $a>1$, we have $\int_{0}^{t} s^{-\frac{1}{a}}(t-s)^{\frac{1}{2 a}-1} d s=c_{a} t^{-\frac{1}{2 a}}$ for some $c_{a}>0$, so $I_{2}$ is in $L^{1}(0, t)$ with

$$
\int_{0}^{t} I_{2}(s) d s \leq C_{a} t^{-\frac{1}{a}} \int_{S_{t}} z^{\frac{1}{a}} y^{\frac{1}{a}} e^{\frac{-(y+z)^{2}}{2 a t}} d y
$$

where $C_{a}>0$ is some numerical constant depending only on $a$.

Step 3. In order to estimate the first term, $I_{1}$, we rely on the inequality

$$
x\left(\frac{2 x y}{t-s} \wedge 1\right)^{a} \leq 4 y \frac{|x-y|^{2}}{t-s}+2 y \text { for any } a>1 .
$$

To see that this inequality is true, simply note that, when $x \leq y$, we have

$$
x\left(\frac{2 x y}{t-s} \wedge 1\right)^{a} \leq x \leq y
$$

while, for $y \leq x$, we can write $x=(x-y)+y$ to obtain

$$
x\left(\frac{2 x y}{t-s} \wedge 1\right)^{a} \leq 2\left(|x-y|^{2}+y^{2}\right)\left(\frac{2 y}{t-s} \wedge \frac{1}{y}\right) \leq 2|x-y|^{2} \frac{2 y}{t-s}+2 y .
$$

Now, using the inequality 6.23 as well as 6.16 from Lemma 6.7, it follows that

$$
\begin{aligned}
I_{1}(s) & :=s^{-\frac{1}{2 a}}(t-s)^{-\frac{1}{2}} \int_{S_{t}}\left(\int_{0}^{\infty}\left|\left(\frac{2 x y}{t-s} \wedge 1\right) \frac{|y-x|}{t-s} e^{-\frac{(y-x)^{2}}{2(t-s)}}\right|^{a} \frac{x z}{s} e^{-\frac{(x-z)^{2}}{2 s}} d x\right)^{\frac{1}{a}} d y \\
& =\frac{1}{t-s} s^{-\frac{3}{2 a}} \int_{S_{t}}\left(\int_{0}^{\infty} x z\left(\frac{2 x y}{t-s} \wedge 1\right)^{a}\left|\frac{y-x}{\sqrt{t-s}}\right|^{a} e^{-\frac{a(y-x)^{2}}{2(t-s)}} e^{-\frac{(x-z)^{2}}{2 s}} d x\right)^{\frac{1}{a}} d y \\
& \lesssim \frac{1}{t-s} s^{-\frac{3}{2 a}} \int_{S_{t}} z^{\frac{1}{a}} y^{\frac{1}{a}} e^{-\frac{(y-z)^{2}}{2 a t}}\left(\int_{0}^{\infty} F(s, x, y, z) d x\right)^{\frac{1}{a}} d y
\end{aligned}
$$


where

$$
F(s, x, y, z):=\left(\left|\frac{y-x}{\sqrt{t-s}}\right|^{a}+\left|\frac{y-x}{\sqrt{t-s}}\right|^{a+2}\right) e^{-\frac{t}{2 s}\left(\frac{(y-x)}{\sqrt{t-s}}+\frac{\sqrt{t-s}(y-z)}{t-s+s a}\right)^{2}} .
$$

For the inner integral, we make the change of variables

$$
w=t^{\frac{1}{2}} s^{-\frac{1}{2}}\left(\frac{(x-y)}{\sqrt{t-s}}+\frac{\sqrt{t-s}(y-z)}{t-s+a s}\right), \quad d x=(t-s)^{\frac{1}{2}} t^{-\frac{1}{2}} s^{\frac{1}{2}} d w
$$

and set

$$
f(w)=f(s, w, y, z):=\left|t^{-\frac{1}{2}} s^{\frac{1}{2}} w-\frac{\sqrt{t-s}(y-z)}{t-s+a s}\right| .
$$

This yields

$$
I_{1}(s) \lesssim s^{-\frac{1}{a}}(t-s)^{\frac{1}{2 a}-1} t^{-\frac{1}{2 a}} \int_{S_{t}} z^{\frac{1}{a}} y^{\frac{1}{a}} e^{-\frac{(y-z)^{2}}{2 a t}}\left(\int_{\mathbb{R}}\left(f(w)^{a}+f(w)^{a+2}\right) e^{-\frac{w^{2}}{2}} d w\right)^{\frac{1}{a}} d y .
$$

Noting that $f(w) \leq|w|+t^{-1}(t-s)^{\frac{1}{2}}|y-z|$, we can split up $I_{1}$ accordingly. Since

$$
\begin{gathered}
t^{-\frac{1}{2 a}} \int_{0}^{t} s^{-\frac{1}{a}}(t-s)^{\frac{1}{2 a}-1} d s=c_{a} t^{-\frac{1}{a}}, \quad t^{-1-\frac{1}{2 a}} \int_{0}^{t} s^{-\frac{1}{a}}(t-s)^{\frac{1}{2 a}-\frac{1}{2}} d s=c_{a}^{\prime} t^{-\frac{1}{2}-\frac{1}{a}} \\
\text { and } t^{-1-\frac{5}{2 a}} \int_{0}^{t} s^{-\frac{1}{a}}(t-s)^{\frac{3}{2 a}-\frac{1}{2}} d s=c_{a}^{\prime \prime} t^{-\frac{1}{2}-\frac{2}{a}}
\end{gathered}
$$

we thus obtain that

$$
\begin{gathered}
\int_{0}^{t} I_{1}(s) d s \lesssim C_{a} t^{-\frac{1}{a}} \int_{S_{t}} z^{\frac{1}{a}} y^{\frac{1}{a}} e^{-\frac{(y-z)^{2}}{2 a t}} d y+C_{a}^{\prime} t^{-\frac{1}{2}-\frac{1}{a}} \int_{S_{t}} z^{\frac{1}{a}} y^{\frac{1}{a}} e^{-\frac{(y-z)^{2}}{2 a t}}|y-z| d y \\
+C_{a}^{\prime \prime} t^{-\frac{1}{2}-\frac{2}{a}} \int_{S_{t}} z^{\frac{1}{a}} y^{\frac{1}{a}} e^{-\frac{(y-z)^{2}}{2 a t}}|y-z|^{1+\frac{2}{a}} d y
\end{gathered}
$$

where

$$
\begin{gathered}
C_{a}=c_{a}\left(\int_{\mathbb{R}}\left(|w|^{a}+|w|^{a+2}\right) e^{-\frac{w^{2}}{2}} d w\right)^{\frac{1}{a}}, \quad C_{a}^{\prime}=c_{a}^{\prime}\left(\int_{\mathbb{R}} e^{-\frac{w^{2}}{2}} d w\right)^{\frac{1}{a}}=c_{a}^{\prime} 2^{\frac{1}{2 a}} \pi^{\frac{1}{2 a}} \\
\text { and } C_{a}^{\prime \prime}=c_{a}^{\prime \prime}\left(\int_{\mathbb{R}} e^{-\frac{w^{2}}{2}} d w\right)^{\frac{1}{a}}=c_{a}^{\prime \prime} 2^{\frac{1}{2 a}} \pi^{\frac{1}{2 a}}
\end{gathered}
$$

Step 4. Given the above, we can now combine the estimates for $I_{1}$ and $I_{2}$. However, we first recall the elementary inequalities

$$
\begin{gathered}
|y-z| e^{-\frac{(y-z)^{2}}{2 a t}} \leq C_{a} t^{\frac{1}{2}} e^{-\frac{(y-z)^{2}}{4 a t}}, \quad(y+z) e^{\frac{-(y+z)^{2}}{2 \sigma t}} \leq C_{a} t^{\frac{1}{2}} e^{-\frac{(y+z)^{2}}{4 a t}}, \\
\text { and } t^{-\frac{1}{a}}|y-z|^{1+\frac{2}{\sigma}} e^{-\frac{(y-z)^{2}}{2 a t}} \leq C_{a} t^{\frac{1}{2}} e^{-\frac{(y-z)^{2}}{4 a t}}
\end{gathered}
$$

Using these, and recalling also (6.19), it follows from 6.22 and 6.24 that there exists a constant $C_{a}>0$ such that

$$
\mathbb{P}^{x_{0}}\left(X_{t \wedge \tau_{i}}^{i, N} \in S\right) \leq \int_{S_{t}} G_{t}(y, z) d y+C_{a} e^{\delta x_{0}^{2}} \int_{S_{t}} t^{-\frac{1}{a}} z^{\frac{1}{a}} y^{\frac{1}{a}} e^{-\frac{(y-z)^{2}}{4 a t}} d y .
$$


Step 5. It remains to observe that 6.25 also holds with 1 in place of $t^{-\frac{1}{a}} z^{\frac{1}{a}} y^{\frac{1}{a}}$. To see this, note that in Step 1 we also have $\left|\partial_{s} v(s, z)\right| \lesssim e^{\delta x_{0}^{2}}\left(J_{1}(s)+J_{2}(s)\right)$, where

$$
\left\{\begin{array}{l}
J_{1}(s):=s^{-\frac{1}{2 a}}(t-s)^{-1} \int_{S_{t}}\left(\int_{0}^{\infty}\left|\frac{y-x}{\sqrt{t-s}}\right|^{a} e^{-\frac{a(y-x)^{2}}{2(t-s)}} e^{-\frac{(x-z)^{2}}{2 s}} d x\right)^{\frac{1}{a}} d y \\
J_{2}(s):=s^{-\frac{1}{2 a}}(t-s)^{-1} \int_{S_{t}}\left(\int_{0}^{\infty}\left|\frac{y+x}{\sqrt{t-s}}\right|^{a} e^{-\frac{a(y+x)^{2}}{2(t-s)}} e^{-\frac{(x-z)^{2}}{2 s}} d x\right)^{\frac{1}{a}} d y .
\end{array}\right.
$$

Using Lemma 6.7 and performing the same changes of variables as in Steps 2 and 3, the computations simplify significantly and we obtain the desired bound.

Step 6. Recall that $S_{t}=\Upsilon_{t}(S)$ and $z=\Upsilon_{0}\left(x_{0}\right)$. In view of Step 4 and Step 5, the proof is therefore finished by performing the change of variables $y=\Upsilon_{t}(x)$.

\subsubsection{Estimates on the whole space}

If we ignore the absorption at the boundary, then the estimates are much simpler, and we get the following bound.

Proposition 6.9. For every $\delta>0$ there exists a $>1$ such that, for every $S \in \mathcal{B}(\mathbb{R})$, it holds uniformly in $N \geq 1$ that

$$
\mathbb{P}^{x_{0}}\left(X_{t}^{i, N} \in S\right) \leq \int_{S} p_{t}\left(\bar{\Upsilon}_{t}\left(x, x_{0}\right)\right) \partial_{x} \bar{\Upsilon}_{t}\left(x, x_{0}\right) d x+C_{a} \int_{S} e^{-\frac{\left(\bar{\Upsilon}_{t}\left(x, x_{0}\right)\right)^{2}}{4 a t}} \partial_{x} \bar{\Upsilon}_{t}\left(x, x_{0}\right) d x
$$

where $p_{t}(x)=(2 \pi t)^{-\frac{1}{2}} \exp \left\{x^{2} / 2 t\right\}$ and $\bar{\Upsilon}_{t}\left(x, x_{0}\right)=\int_{x_{0}}^{x} \sigma(t, y)^{-1} d y$.

Proof. Consider Lemma 6.3 with $\bar{Z}_{t}:=\bar{\Upsilon}_{t}\left(X_{t}^{i}, x_{0}\right)$ in place of $Z_{t}=\Upsilon_{t}\left(X_{t}^{i}\right)=\bar{\Upsilon}_{t}\left(X_{t}^{i}, 0\right)$ and let $\overline{\mathbb{Q}}$ denote the corresponding measure from Lemma 6.4 such that $\bar{Z}_{t}$ is a standard Brownian motion under $\overline{\mathbb{Q}}$, started at 0 when $X_{0}^{i}=x_{0}$. Setting $\bar{S}_{t}:=\bar{\Upsilon}_{t}\left(S, x_{0}\right)$, we have

$$
\mathbb{P}^{x_{0}}\left(X_{t}^{i, N} \in S\right)=\mathbb{P}^{0}\left(\bar{Z}_{t} \in \bar{S}_{t}\right) .
$$

From here, the bound follows by analogy with Proposition 6.8 for the new measure $\overline{\mathbb{Q}}$ and

$$
\bar{v}(s):=\mathbb{E}^{0}\left[\bar{u}\left(s, \bar{Z}_{s}\right)\right], \quad \bar{u}(s, x):=\mathbb{P}^{x}\left(W_{t-s} \in \bar{S}_{t}\right)=\int_{\bar{S}_{t}} p_{t-s}(y-x) d y .
$$

Since there are no boundary effects, the estimates simplify and, in fact, the work is the same as for $J_{1}$ in Step 5 of the proof of Proposition 6.8 with $z=0$.

\subsection{Proof of Proposition 3.3}

In view of Propositions 6.8 and 6.9 , only a few observations remain before we can deduce the density estimates $(3.2)$ and $(3.3)$, thus proving Proposition 3.3 Given Assumption 2.1 . it holds by construction of $\bar{\Upsilon}$ that there are constants $C_{1}, C_{2}>0$ such that $C_{1}\left|x-x_{0}\right| \leq$ $\left|\bar{\Upsilon}_{t}\left(x, x_{0}\right)\right| \leq C_{2}\left|x-x_{0}\right|$ and $\left|\partial_{x} \Upsilon_{t}(x)\right| \leq C_{2}$ for all $x \in \mathbb{R}$ and $t \in[0, T]$. Consequently, the whole-space estimate 3.3$)$ is an immediate consequence of Proposition 6.9.

For the estimate with boundary decay, we begin by recalling the standard bound

$$
G_{t}\left(x, x_{0}\right) \leq C \frac{1}{\sqrt{t}}\left(\frac{x}{\sqrt{t}} \wedge 1\right)\left(\frac{x_{0}}{\sqrt{t}} \wedge 1\right) \exp \left\{\frac{-\left(x-x_{0}\right)^{2}}{4 t}\right\} \text { for } \quad x, x_{0} \geq 0 .
$$


Next, we notice that, by definition of $\Upsilon$, there exist $C>0$ such that $\left|\Upsilon_{t}(x)\right| \leq C x$ and $\left|\partial_{x} \Upsilon_{t}(x)\right| \leq C$ for all $x \geq 0$ and $t \in[0, T]$. Finally, we can observe that

$$
\begin{aligned}
\left(\Upsilon_{t}(x)-\Upsilon_{0}\left(x_{0}\right)\right)^{2} & \geq\left(\int_{x \wedge x_{0}}^{x \vee x_{0}} \frac{d y}{\sigma(t, y)}\right)^{2}+2 \int_{x \wedge x_{0}}^{x \vee x_{0}} \frac{d y}{\sigma(t, y)} \int_{0}^{x \wedge x_{0}} \frac{1}{\sigma(t, y)}-\frac{1}{\sigma(0, y)} d y \\
& \geq C\left(x-x_{0}\right)^{2}-C^{\prime} t\left|x-x_{0}\right|\left(x \wedge x_{0}\right),
\end{aligned}
$$

by using the bounds on $\sigma$ and $\partial_{t} \sigma$ from Assumption 2.1. Combining these observations, the density estimate 3.2 follows from Proposition 6.8 by taking $c_{x, y}:=4 a C^{\prime}|x-y|(x \wedge y)$ and $\kappa:=1 / a$.

It remains to observe that we can take $c_{x, y} \equiv 0$ when $\sigma(t, x)=\sigma_{1}(t) \sigma_{2}(x)$ and that, in this case, we do not need any smoothness of $t \mapsto \sigma(t, x)$, as mentioned in Remark 2.2

To see this, the point is that it suffices to scale away the spatial component of the volatility. Specifically, we can consider the analogue of Lemma 5.2 with

$$
\tilde{\Upsilon}(x):=\int_{0}^{x} \frac{d y}{\sigma_{2}(y)} \quad \text { and } \quad \tilde{Z}_{t}^{i}:=\tilde{\Upsilon}\left(X_{t}^{i}\right)=\tilde{\Upsilon}\left(X_{0}^{i}\right)+\int_{0}^{t} \tilde{b}_{s}^{i} d s+\int_{0}^{t} \sigma_{1}(s) d B_{s} .
$$

Fix $S \in \mathcal{B}(0, \infty)$ and set $\tilde{S}:=\tilde{\Upsilon}(S)$. Fixing also $t \in(0, T]$, we can replicate Section 6.3 with $\tilde{v}(s, z):=\mathbb{E}^{z}\left[\tilde{u}\left(s, \tilde{Z}_{s \wedge \tilde{\tau}}\right)\right]$ and

$$
\tilde{u}(s, x):=\mathbb{P}^{x}\left(W_{\int_{s}^{t} \sigma_{1}(r)^{2} d r} \in \tilde{S}, \int_{s}^{t} \sigma_{1}(r)^{2} d r<\tau_{W}\right)=\int_{\tilde{S}} G_{s, t}^{\sigma_{1}}(y, x) d y,
$$

where $G_{s, t}^{\sigma_{1}}$ is the Green's function for $\partial_{s} f(s, x)+\frac{1}{2} \sigma_{1}(s)^{2} \Delta f(s, x)=0$ as a terminalboundary value problem on $[0, t) \times(0, \infty)$ with $f(s, 0)=0$. That is,

$$
G_{s, t}^{\sigma_{1}}(y, x)=\left(2 \pi \int_{s}^{t} \sigma_{1}(r)^{2} d r\right)^{-\frac{1}{2}}\left(\exp \left\{\frac{-(x-y)^{2}}{2 \int_{s}^{t} \sigma_{1}(r)^{2} d r}\right\}-\exp \left\{\frac{-(x+y)^{2}}{2 \int_{s}^{t} \sigma_{1}(r)^{2} d r}\right\}\right) .
$$

Since there are constants $c_{1}, c_{2}>0$ such that $c_{1}(t-s) \leq \int_{s}^{t} \sigma_{1}(r)^{2} d r \leq c_{2}(t-s)$, the same estimates as in the proof of Proposition 6.8 yield

$$
\mathbb{P}^{x_{0}}\left(X_{t \wedge \tau_{i}}^{i} \in S\right)=\int_{\tilde{S}} G_{s, t}^{\sigma_{1}}\left(y, \tilde{\Upsilon}\left(x_{0}\right)\right) d y+\int_{0}^{t} \partial_{s} \tilde{v}\left(s, \tilde{\Upsilon}\left(x_{0}\right)\right) d s
$$

and, using also the bounds $|\tilde{\Upsilon}(x)| \leq c x$ and $\left|\partial_{x} \tilde{\Upsilon}\right| \leq c$, we get

$$
\mathbb{P}^{x_{0}}\left(X_{t \wedge \tau_{i}}^{i} \in S\right) \lesssim \int_{S} G_{0, t}^{\sigma_{1}}\left(\tilde{\Upsilon}(x), \tilde{\Upsilon}\left(x_{0}\right)\right) d x+e^{\delta x_{0}^{2}} \int_{S}\left(t^{-\frac{1}{a}} x^{\frac{1}{a}} x_{0}^{\frac{1}{a}} \wedge 1\right) e^{-\frac{\left(\tilde{\Upsilon}(x)-\tilde{\Upsilon}\left(x_{0}\right)\right)^{2}}{4 a t}} d x
$$

As $\tilde{\Upsilon}$ does not depend on time, we have $\left|\tilde{\Upsilon}(x)-\tilde{\Upsilon}\left(x_{0}\right)\right|=\left|\int_{x_{0}}^{x} \sigma_{2}(y)^{-1} d y\right|$, so there exist $c_{1}^{\prime}, c_{2}^{\prime}>0$ such that $c_{1}^{\prime}\left|x-x_{0}\right| \leq\left|\tilde{\Upsilon}(x)-\tilde{\Upsilon}\left(x_{0}\right)\right| \leq c_{2}^{\prime}\left|x-x_{0}\right|$. Also, $G_{0, t}^{\sigma_{1}}$ satisfies an analogous bound to that of $G_{t}(x, y)$ above, so we conclude that the density estimate 3.2 holds with $c_{x, y} \equiv 0$, as desired. This finishes the proof of Proposition 3.3 . 


\section{A Appendix}

\section{A.1 Technical lemmas}

Lemma A.1. Suppose $\nu$ satisfies Assumption 2.3 and let $g_{s}(x)=g\left(s, x, \nu_{s}, L_{s}\right)$, where $g$ is any of $b, \mathfrak{b}, \sigma$, or $\sigma^{2}$, with $L_{s}=1-\nu_{s}(0, \infty)$. Define the error term

$$
\mathcal{E}_{t, \varepsilon}^{g}(x):=\left\langle\nu_{t}, g_{t}(\cdot) G_{\varepsilon}(x, \cdot)\right\rangle-g_{t}(x)\left(\mathcal{T}_{\varepsilon} \nu_{t}\right)(x) .
$$

Then we have

$$
\mathbb{E} \int_{0}^{T}\left\|\mathcal{E}_{t, \varepsilon}^{g}\right\|_{L^{2}(\mathbb{R})}^{2} d t \rightarrow 0 \quad \text { as } \quad \varepsilon \rightarrow 0 .
$$

Proof. This follows by a straightforward modification of Lemma 8.1 in [35. The only thing to note is that we can no longer use the crude bound $\left|g_{t}(x)-g_{t}(y)\right| \leq 2\left\|g_{t}\right\|_{\infty}$, as $g_{t}$ need not be bounded. However, the arguments from [35] are easily extended to the present case if we instead rely on $\left|g_{t}(x)-g_{t}(y)\right| \leq\left\|\partial_{x} g_{t}\right\|_{\infty}|x-y|$.

Lemma A.2. Suppose $\bar{\nu}$ satisfies the whole-space analogues of (iii)-(iv) in Assumption 2.3. Let $g_{s}=g\left(s, \cdot, \nu_{s}, L_{s}\right)$, where $g$ is any of $b, \mathfrak{b}, \sigma$, or $\sigma^{2}$, and define the error terms

$$
\begin{aligned}
\overline{\mathcal{E}}_{t, \varepsilon}^{g}(x) & :=\left\langle\bar{\nu}_{t}, g_{t}(\cdot) p_{\varepsilon}(x, \cdot)\right\rangle-g_{t}(x) \partial_{x}\left(\overline{\mathcal{T}}_{\varepsilon} \bar{\nu}_{t}\right)(x)+\partial_{x} g_{t}(x) \overline{\mathcal{H}}_{t, \varepsilon}^{g}(x), \\
\overline{\mathcal{H}}_{t, \varepsilon}^{g}(x) & :=\left\langle\bar{\nu}_{t},(x-\cdot) \partial_{x} p_{\varepsilon}(x-\cdot)\right\rangle .
\end{aligned}
$$

Then, for $k=1,2$, we have

$$
\mathbb{E} \int_{0}^{T}\left\|\overline{\mathcal{E}}_{t, \varepsilon}^{g}\right\|_{L^{2}(\mathbb{R})}^{2 k} d t \rightarrow 0, \quad \mathbb{E} \int_{0}^{T}\left\|x \overline{\mathcal{E}}_{t, \varepsilon}^{g}\right\|_{L^{2}(\mathbb{R})}^{2} d t \rightarrow 0, \quad \text { as } \quad \varepsilon \rightarrow 0 .
$$

Proof. This follows by a simple modification of Lemma 8.2 in 35 .

Lemma A.3. Let $\nu_{t}^{N}$ be as defined in 2.1) and let $\tilde{\nu}_{t}$ be any measure valued process satisfying Assumption 2.3. Then, for every a $>0$, we have

$$
\mathbb{E} \int_{0}^{T}\left\langle\tilde{\nu}_{t}, x^{k} \mathbf{1}_{[\lambda, \infty)}(x)\right\rangle d t=o\left(\lambda^{k} e^{-a \lambda}\right) \quad \text { as } \quad \lambda \rightarrow \infty,
$$

and, likewise, it holds uniformly in $N \geq 1$ and $t \in[0, T]$ that

$$
\mathbb{E}\left\langle\nu_{t}^{N}, x^{k} \mathbf{1}_{[\lambda, \infty)}(x)\right\rangle=o\left(\lambda^{k} e^{-a \lambda}\right) \quad \text { as } \quad \lambda \rightarrow \infty
$$

Proof. Fix an arbitrary $a>0$ and let $\varepsilon>0$ be given. By Corollary 3.4 , there exists $\lambda_{0} \geq 0$ such that

$$
\mathbb{E}\left[\nu_{t}^{N}(\lambda, \infty)\right] \leq \varepsilon e^{-a \lambda} \quad \forall \lambda \geq \lambda_{0},
$$

uniformly in $N \geq 1$ and $t \in[0, T]$. Given any $\lambda \geq \lambda_{0}$ we let $\left\{s_{0}, s_{1}, \ldots\right\}$ denote the partition of $[\lambda, \infty)$ with $s_{i}-s_{i-1}=1 / a$. By A.3 and monotone convergence, we get

$$
\mathbb{E}\left\langle\nu_{t}^{N}, x \mathbf{1}_{[\lambda, \infty)}(x)\right\rangle \leq \sum_{i=1}^{\infty} s_{i} \mathbb{E} \nu_{t}^{N}\left(s_{i-1}, \infty\right) \leq \varepsilon \sum_{i=1}^{\infty} s_{i} e^{-a s_{i-1}}=\varepsilon e \sum_{i=1}^{\infty} s_{i} e^{-a s_{i}}
$$


Now, $x \mapsto x e^{-a x}$ is decreasing for $x \geq 1 / a$, so taking $\lambda_{0}^{\prime}:=\max \left\{1 / a, \lambda_{0}\right\}$ and noting that $s_{0}=\lambda$, it holds for all $\lambda>\lambda_{0}^{\prime}$ that

$$
\mathbb{E}\left\langle\nu_{t}^{N}, x \mathbf{1}_{[\lambda, \infty)}(x)\right\rangle \leq \varepsilon e \int_{\lambda}^{\infty} x e^{-a x} d x=\varepsilon e\left(\frac{\lambda}{a}+\frac{1}{a^{2}}\right) e^{-a \lambda} .
$$

This proves A.2 for $k=1$ and the work for $k \geq 2$ is analogous. The claim in A.1 follows similarly, by relying on the exponential tail property from Assumption 2.3 .

Lemma A.4. Let $\nu$ be a limit point from Theorem 2.4 and let $\tilde{\nu}$ be any measure valued process satisfying Assumption 2.3. Then it holds with probability 1 that $($ as $\lambda \rightarrow \infty)$,

$$
\lim _{\varepsilon \downarrow 0} \frac{\nu_{t}(0, \varepsilon)}{\varepsilon}=0, \quad \nu_{t}(\lambda, \infty)=O\left(e^{-\lambda}\right), \quad \text { and } \quad \int_{0}^{T} \tilde{\nu}_{t}(\lambda, \infty) d t=O\left(e^{-\lambda}\right) .
$$

Proof. For the first claim, we recall from Proposition 4.3 that there exists $\delta \in(0,1]$ and $\beta>0$ such that $\mathbb{E} \nu_{t}(0, \varepsilon)=t^{-\frac{\delta}{2}} O\left(\varepsilon^{1+\beta}\right)$ as $\varepsilon \rightarrow 0$. Using Markov's inequality, we thus deduce that, for any $\theta>0$ and $n$ sufficiently large,

$$
\mathbb{P}\left(n^{2 / \beta} \nu_{t}\left(0, n^{-2 / \beta}\right)>\theta\right) \leq \theta^{-1} n^{2 / \beta} \mathbb{E} \nu_{t}\left(0, n^{-2 / \beta}\right) \leq C t^{-\frac{\delta}{2}} \theta^{-1} n^{-2} .
$$

Hence the Borel-Cantelli lemma gives $\lim \sup _{n} n^{2 / \beta} \nu_{t}\left(0, n^{-2 / \beta}\right)=0$ with probability 1 . Now, given $\varepsilon>0$, we have $(n+1)^{-2 / \beta}<\varepsilon \leq n^{-2 / \beta}$ for some $n \geq 1$, so we deduce that

$$
\limsup _{\varepsilon \downarrow 0} \frac{\nu_{t}(0, \varepsilon)}{\varepsilon} \leq \limsup _{n \geq 1} \frac{\nu_{t}\left(0, n^{-2 / \beta}\right)}{(n+1)^{-2 / \beta}} \leq \limsup _{n \geq 1} \frac{\nu_{t}\left(0, n^{-2 / \beta}\right)}{n^{-2 / \beta}}\left(\frac{n+1}{n}\right)^{\frac{2}{\beta}} .
$$

Since the latter is zero with probability 1 , this proves the first claim. The two remaining results follow by analogous considerations for the tail probabilities, using the exponential tail properties from Proposition 4.3 and Assumption 2.3 respectively.

\section{A.2 Proofs of Propositions 3.5 and 3.6}

Lemma A.5. Let $\Lambda_{t}^{i, N}=\left|X_{t}^{i}\right|+\sum_{j=1}^{N} a_{j}^{N}\left|X_{t}^{j}\right|$, as in 6.1.). Then it holds uniformly in $N \geq 1$ that

$$
\mathbb{P}\left(\sup _{t \leq T} \Lambda_{t}^{i, N} \geq \lambda\right)=o(1) \quad \text { as } \quad \lambda \rightarrow \infty .
$$

Proof. This is an immediate consequence of Corollary 6.2 .

Based on this lemma, we can adapt the arguments from Section 4 of [35] to prove Proposition 3.5 and Proposition 3.6.

Proof of Proposition 3.5. Arguing as in the proof of Proposition 4.6 in [35], for all $a>0$ and $\theta:=\frac{1}{2}(1-r)$, we have

$$
\mathbb{P}\left(L_{t+h}^{N}-L_{t}^{N}<\delta, L_{t}^{N}<r\right) \leq \mathbb{P}\left(L_{t+h}^{N}-L_{t}^{N}<\delta, \nu_{t}^{N}(0, a)>\theta\right)+o\left(e^{-a}\right) .
$$

Let $E:=\left\{L_{t+h}^{N}-L_{t}^{N}<\delta, \nu_{t}^{N}(0, a)>\theta\right\}$ and define a random index set $\mathcal{I}$ by

$$
\mathcal{I}:=\left\{1 \leq i \leq N: X_{t}^{i}<a, t<\tau_{i}, a_{i}^{N}>\theta / 2 N\right\} .
$$


Note that the particles with index in $\left\{1 \leq i \leq N: a_{i}^{N} \leq \frac{1}{2} \theta N^{-1}\right\}$ can contribute at most $\frac{1}{2} \theta$ towards $\nu_{t}^{N}(0, a)$. Recalling also that $a_{i}^{N} \leq m / N$ for some $m>0$, see 2.2 , it follows that on the event $E$ we must have $|\mathcal{I}|>N \theta / 2 m$. Hence

$$
\mathbb{P}(E) \leq \sum_{\mathcal{I}_{0}:\left|\mathcal{I}_{0}\right|>\frac{\theta N}{2 m}} \mathbb{P}\left(E \mid \mathcal{I}=\mathcal{I}_{0}\right) \mathbb{P}\left(\mathcal{I}=\mathcal{I}_{0}\right) .
$$

Moreover, since $L_{t+h}^{N}-L_{t}^{N}<\delta$ on $E$ while $a_{i}^{N}>\theta / 2 N$ for $i \in \mathcal{I}$, we deduce that

$$
\mathbb{P}\left(E \mid \mathcal{I}=\mathcal{I}_{0}\right) \leq \mathbb{P}\left(\#\left\{i \in \mathcal{I}_{0}: \inf _{u \leq h} X_{t+u}^{i} \leq 0\right\}<2 \delta N / \theta \mid \mathcal{I}=\mathcal{I}_{0}\right) .
$$

In order to estimate A.5, we let $Z_{t}^{i}=\Upsilon_{t}\left(X_{t}^{i}\right)$ as in Lemma 6.3 and recall that $Z_{t}^{i}$ then satisfies

$$
d Z_{t}^{i}=\hat{b}_{t}^{i} d t+d B_{t}^{i}, \quad\left|\hat{b}_{t}^{i}\right| \leq c_{1}\left(1+\Lambda_{t}^{i, N}\right), \quad\left|Z_{t}^{i}\right| \leq c\left(1+\left|X_{t}^{i}\right|\right) .
$$

Using that $Z_{t}^{i} \leq 0$ if and only if $X_{t}^{i} \leq 0$, the estimate in A.5 implies

$$
\mathbb{P}\left(E \mid \mathcal{I}_{0}=\mathcal{I}\right) \leq \mathbb{P}\left(\#\left\{i \in \mathcal{I}: \inf _{u \leq h} Z_{t+u}^{i} \leq 0\right\}<2 \delta N / \theta \mid \mathcal{I}_{0}=\mathcal{I}\right) .
$$

Moreover, it is immediate from that

$$
Z_{t+u}^{i} \leq Z_{t}^{i}+c_{1} h+\sup _{u \leq h} \Lambda_{t+u}^{i, N} c_{1} h+\tilde{B}_{u}^{i}, \quad \tilde{B}_{u}^{i}:=B_{t+u}^{i}-B_{t}^{i}, \quad \tilde{\Lambda}_{h}^{i, N}:=\sup _{u \leq h} \Lambda_{t+u}^{i, N} .
$$

In particular, if $\inf _{u \leq h} \tilde{B}_{u}^{i} \leq-Z_{t}^{i}-c_{1} h\left(1+\tilde{\Lambda}_{h}^{i, N}\right)$, then $\inf _{u \leq h} Z_{t+u}^{i} \leq 0$, and hence

$$
\mathbb{P}\left(E \mid \mathcal{I}_{0}=\mathcal{I}\right) \leq \mathbb{P}\left(\#\left\{i \in \mathcal{I}: \inf _{u \leq h} \tilde{B}_{u}^{i} \leq-Z_{t}^{i}-c_{1} h\left(1+\tilde{\Lambda}_{h}^{i, N}\right)\right\}<\frac{2}{\theta} \delta N \mid \mathcal{I}_{0}=\mathcal{I}\right) .
$$

Using the bound $\left|Z_{t}^{i}\right| \leq c\left|X_{t}^{i}\right|$ from A.6), the definition of $\mathcal{I}$ implies that there exists $c_{2}>0$ such that

$$
\mathbb{P}\left(E \mid \mathcal{I}_{0}=\mathcal{I}\right) \leq \mathbb{P}\left(\#\left\{i \in \mathcal{I}: \inf _{u \leq h} \tilde{B}_{u}^{i} \leq-c_{2} a-c_{2}\left(1+\tilde{\Lambda}_{h}^{i, N}\right)\right\}<\frac{2}{\theta} \delta N / \mid \mathcal{I}_{0}=\mathcal{I}\right) .
$$

Recalling the fact that

$$
\tilde{B}_{u}^{i}=\int_{t}^{t+u} \rho_{s} d W_{s}^{0}+\int_{t}^{t+u} \sqrt{1-\rho_{s}^{2}} d W_{s}^{i}=: I_{u}+J_{u}^{i},
$$

we split the above probability on the event $\left\{\sup _{u \leq h}\left|I_{u}\right|<c_{2} a\right\} \cap\left\{\sup _{u \leq h} \Lambda_{t+u}^{i, N}<a\right\}$ and its complement. In this way, we get

$$
\begin{gathered}
\mathbb{P}\left(E \mid \mathcal{I}_{0}=\mathcal{I}\right) \leq \mathbb{P}\left(\#\left\{i \in \mathcal{I}: \inf _{u \leq h} J_{u}^{i} \leq-3 c_{2} a-c_{2}\right\}<\frac{2}{\theta} \delta N \mid \mathcal{I}_{0}=\mathcal{I}\right) \\
+\mathbb{P}\left(\sup _{u \leq h}\left|I_{u}\right| \geq c_{2} a\right)+\mathbb{P}\left(\sup _{u \leq h} \Lambda_{t+u}^{i, N} \geq a\right) .
\end{gathered}
$$

From Lemma A.5 we know that the last term is $o(1)$ as $a \rightarrow \infty$ uniformly in $N \geq 1$. Similarly, $I_{u}$ is a martingale, and hence the second term is also $o(1)$ as $a \rightarrow \infty$ by Doob's Maximal Inequality. Concerning the first term, we can introduce a time-change to make each $J^{i}$ an independent Brownian motion. Recalling A.4, the proof can then be completed via a law of large numbers argument as in Proposition 4.6 of [35], by carefully choosing the free parameter $a$ as a function of $\delta$. 
Proof of Proposition 3.6. Arguing as in Proposition 4.7 of [35], it suffices to show that

$$
\lim _{\delta \rightarrow 0} \lim _{N \rightarrow \infty} \mathbb{P}\left(L_{t+\delta}^{N}-L_{t}^{N} \geq \eta, \nu_{t}^{N}(0, \varepsilon)<\eta / 2\right)=0 .
$$

Let $E:=\left\{L_{t+\delta}^{N}-L_{t}^{N} \geq \eta, \nu_{t}^{N}(0, \varepsilon)<\eta / 2\right\}$ and define the random index set

$$
\mathcal{I}:=\left\{1 \leq i \leq N: X_{t}^{i} \geq \varepsilon \text { or } t \geq \tau_{i}\right\} .
$$

By (2.2), there exists $m$ such that $a_{i}^{N} \leq m / N$, so noting that $\left\{\nu_{t}^{N}(0, \varepsilon)<\eta / 2\right\}$ is contained in $\left\{|\mathcal{I}| \geq \frac{N}{m}\left(1-\frac{\eta}{2}\right)\right\}$, we have

$$
\mathbb{P}(E) \leq \sum_{\mathcal{I}_{0}:\left|\mathcal{I}_{0}\right| \geq \frac{N}{m}\left(1-\frac{\eta}{2}\right)} \mathbb{P}\left(E \mid \mathcal{I}=\mathcal{I}_{0}\right) \mathbb{P}\left(\mathcal{I}=\mathcal{I}_{0}\right)
$$

The conditional probabilities can be estimated by

$$
\begin{aligned}
\mathbb{P}\left(E \mid \mathcal{I}=\mathcal{I}_{0}\right) & \leq \mathbb{P}\left(\#\left\{i \in \mathcal{I}_{0}: \inf _{s \in[t, t+\delta]} X_{s}^{i} \leq 0, \quad X_{t}^{i} \geq \varepsilon\right\} \geq \frac{N \eta}{2 m} \mid \mathcal{I}=\mathcal{I}_{0}\right) \\
& \leq \mathbb{P}\left(\#\left\{i \in \mathcal{I}_{0}: \inf _{s \in[t, t+\delta]}\left(X_{s}^{i}-X_{t}^{i}\right) \leq-\varepsilon\right\} \geq \frac{N \eta}{2 m} \mid \mathcal{I}=\mathcal{I}_{0}\right)
\end{aligned}
$$

Using the scale transform $\Upsilon$ from Lemma 6.3, we introduce $U_{s}^{i}:=\Upsilon_{t+s}\left(X_{t+s}^{i}-X_{t}^{i}\right)$ and note that, as in Lemma 6.3 .

$$
d U_{s}^{i}=u_{s}^{i} d s+\rho_{t+s} d W_{t+s}^{0}+\left(1-\rho_{t+s}^{2}\right)^{\frac{1}{2}} d W_{t+s}^{i}=: u_{s}^{i} d s+d I_{s}+d J_{s}^{i}
$$

where the drift satisfies $\left|u_{s}^{i}\right| \leq c_{1}\left(1+\Lambda_{t+s}^{i, N}\right)$. By construction of $\Upsilon$ and the boundedness from below of $1 / \sigma$, say $1 / \sigma \geq c_{2}>0$, it follows from A.7 that

$$
\mathbb{P}\left(E \mid \mathcal{I}=\mathcal{I}_{0}\right) \leq \mathbb{P}\left(\#\left\{i \in \mathcal{I}_{0}: \inf _{s \leq \delta} U_{s}^{i} \leq-c_{2} \varepsilon\right\} \geq \frac{N \eta}{2 m} \mid \mathcal{I}=\mathcal{I}_{0}\right) .
$$

Given $\delta>0$, fix a constant $a=a(\delta)>0$ to be specified later. Using the decomposition A.8) of $U_{s}^{i}$ and the growth estimate for its drift $u_{s}^{i}$, we see that, on the event

$$
\left\{\sup _{s \leq \delta} \Lambda_{t+s}^{i, N}<a / \delta\right\} \cap\left\{\sup _{s \leq \delta}\left|I_{s}\right|<a\right\}
$$

if $i \in \mathcal{I}_{0}$ is such that $\inf _{s \leq \delta} U_{s}^{i} \leq-c_{2} \varepsilon$, then

$$
\begin{aligned}
\inf _{s \leq \delta} J_{s}^{i} & \leq-c_{2} \varepsilon+\delta c_{1}\left(1+\sup _{s \leq \delta} \Lambda_{t+s}^{i, N}\right)+\sup _{s \leq \delta}\left|I_{s}\right| \\
& \leq-c_{2} \varepsilon+c_{1} \delta+\left(1+c_{1}\right) a \leq-c_{3}(\varepsilon-\delta-a)
\end{aligned}
$$

Consequently, splitting up the desired probability on the event A.9 and its complement,

$$
\begin{array}{r}
\mathbb{P}\left(E \mid \mathcal{I}=\mathcal{I}_{0}\right) \leq \mathbb{P}\left(\#\left\{i \in \mathcal{I}_{0}: \inf _{s \leq \delta} J_{s}^{i} \leq-c_{3}(\varepsilon-\delta-a)\right\} \geq \frac{N \eta}{2 m} \mid \mathcal{I}=\mathcal{I}_{0}\right) \\
+\mathbb{P}\left(\sup _{s \leq \delta} \Lambda_{t+s}^{i, N} \geq a / \delta\right)+\mathbb{P}\left(\sup _{s \leq \delta}\left|I_{s}\right| \geq a\right) .
\end{array}
$$


By Doob's maximal inequality, the last term is bounded by $\delta a^{-2}$, so choosing $a=a(\delta)$ s.t. $\delta a^{-2} \rightarrow 0$ as $\delta \rightarrow 0$, we get

$$
\mathbb{P}\left(\sup _{s \leq \delta}\left|I_{s}\right| \geq a\right)=o(1) \quad \text { as } \quad \delta \rightarrow 0 .
$$

Moreover, ensuring also that $\delta / a \rightarrow 0$ as $\delta \rightarrow 0$, it follows from Lemma A.5 that

$$
\mathbb{P}\left(\sup _{s \leq \delta} \Lambda_{t+s}^{i, N} \geq a / \delta\right)=o(1) \quad \text { as } \quad \delta \rightarrow 0 .
$$

The above requirements are satisfied by $a=a(\delta):=\delta^{1 / 2} \log \log (1 / \delta)$. With this choice for $a$, the proof can now be finished by the same arguments as in Proposition 4.7 of [35].

\section{References}

[1] Acharya, V.V., Pedersen, L.H., Philippon, T., Richardson, M.: Measuring Systemic Risk. Rev. Finan. Stud. 30, 2-47 (2017)

[2] Allen, F., Gale, D.: Financial Contagion. J. Polit. Economy 108, 1-33 (2000)

[3] Azizpour, S., Giesecke, K., Schwenkler, G.: Exploring the Sources of Default Clustering. J. Finan. Econ., forthcoming (2017). Available at SSRN:1127792.

[4] Banerjee, T., Bernstein, A., Feinstein, Z.: Dynamic clearing and contagion in financial networks. Preprint (2018). Available at arXiv:1801.02091.

[5] Benoit, S., Colliard, J.-E., Hurlin, C., Pérignon, C.: Where the Risks Lie: A Survey on Systemic Risk. Review of Finance, 21, 109-152 (2017)

[6] Berg, T., Gider, J.: What Explains the Difference in Leverage between Banks and Non-Banks? J. Finan. Quant. Anal. 52, 2677-2702 (2017)

[7] Brunnermeier, M.K.: Deciphering the Liquidity and Credit Crunch 2007-2008. J. Econ. Perspect. 23, 77-100 (2009)

[8] Brunnermeier, M.K., Gorton, G., Krishnamurthy, A.: Risk Topography. NBER Macroeconomics Annual 26, 149-176 (2012)

[9] Bush, N., Hambly B.M., Haworth, H., Jin, L., Reisinger, C.: Stochastic evolution equations in portfolio credit modelling. SIAM J. Fin. Math. 2, 627-664 (2011)

[10] Capponi, A., Bo, L.: Systemic Risk in Interbanking Networks. SIAM J. Fin. Math. 6, 386-424 (2015)

[11] Carmona, R., Fouque, J.-P., Sun, L.-H.: Mean Field Games and Systemic Risk. Comm. Math. Sci. 13, 911-933 (2015)

[12] Cho, S., Kim, P., and Park, H.: Two-sided estimates on Dirichlet heat kernels for timedependent parabolic operators with singular drifts in $C^{1, \alpha}$-domains. J. Differential Equations 252, 1101-1145 (2012) 
[13] Cochrane, J.H.: Toward a Run-Free Financial System. In: Baily, M.N., Taylor, J.B. (eds.) Across the Great Divide, 197-249. Hoover Press (2014)

[14] Cont, R, Moussa, A., Santos, E.B.: Network structure and systemic risk in banking systems. In: Fouque, J.-P., Langsam, J. (eds.) Handbook of Systemic Risk, 327-368. CUP (2013)

[15] Cont, R., Wagalath, L.: Running for the exit: distressed selling and endogenous correlation in financial markets. Math. Finance 23, 718-741 (2013)

[16] Cont, R., Wagalath, L.: Fire sale forensics: measuring endogenous risk. Math. Finance 26, 835-866 (2016)

[17] Cvitanic, J., Ma, J., Zhang, J.: The law of large numbers for self-exciting correlated defaults. Stoch. Proc. Appl. 122, 2781-2810 (2012)

[18] Danielsson, J., Shin, H.S., Zigrand, J.-P.: Endogenous Extreme Events and the Dual Role of Prices. Annu. Rev. Econ. 4, 111-129 (2012)

[19] Dees, S., Henry, J., Martin, R.: STAMPE: Stress-Test Analytics for Macroprudential Purposes in the Euro Area. ECB (2017)

[20] Delarue, F., Inglis, J., Rubenthaler, S., Tanré, E.: Global solvability of a networked integrate-and-fire model of McKean-Vlasov type. Ann. Appl. Probab. 25, 2096-2133 $(2015)$

[21] Delarue, F., Inglis, J., Rubenthaler, S., Tanré, E.: Particle systems with a singular mean-field self-excitation. Application to neuronal networks. Stoch. Proc. Appl. 125, 2451-2492 (2015)

[22] Duffie, D.: Financial Regulatory Reform After the Crisis: An Assessment. Management Science, Articles in Advance (2017)

[23] Duffie, D., Eckner, A., Horel, G., Saita, L.: Frailty correlated default. J. Finance 64, 2089-2123 (2009)

[24] Eisenberg, L., Noe, T.H.: Systemic risk in financial systems. Management Science 47, $236-249$ (2001)

[25] Fouque, J.-P., Ichiba, T.: Stability in a model of interbank lending. SIAM J. Fin. Math. 4, 784-803 (2013)

[26] Fouque, J.-P., Sun, L.-H. Systemic risk illustrated. In: Fouque, J.-P., Langsam, J. (eds.) Handbook on Systemic Risk, 444-452. CUP (2013)

[27] Garnier, J., Papanicolaou, G., Yang,.: Large deviations for a mean field model of systemic risk. SIAM J. Fin. Math. 4, 151-184 (2013)

[28] Garnier, J., Papanicolaou, G., Yang, T.-W.: A risk analysis for a system stabilized by a central agent. Risk and Decision Analysis 6, 97-120 (2017)

[29] Giesecke, K., Schwenkler, G., Sirignano, J.: Inference for Large Financial Systems. Questrom School of Business Research Paper (2017). Available at SSRN:3012751. 
[30] Giesecke, K., Spiliopoulos, K., Sowers, R.: Default clustering in large portfolios: Typical events. Ann. Appl. Probab. 23, 348-385 (2013)

[31] Giesecke, K., Spiliopoulos, K., Sowers, R., Sirignano, J.: Large portfolio asymptotics for loss from default. Math. Finance 25, 77-114 (2015)

[32] Glasserman, P., Young, H.P.: Contagion in Financial Networks. J. Econ. Literature 54, 779-831 (2016)

[33] Gornall, W., Strebulaev, I.A.: Financing as a Supply Chain: The Capital Structure of Banks and Borrowers. Working Paper (2017). Available at SSRN:2347107.

[34] Gyrya, P., Saloff-Coste L.: Neumann and Dirichlet Heat Kernels in Inner Uniform Domains. Astérisque 336. Société Mathématique de France (2011)

[35] Hambly, B.M., Ledger, S.: A Stochastic McKean-Vlasov Equation for Absorbing Diffusions on the Half-Line. Ann. Appl. Probab. 27, 2698-2752 (2017)

[36] Hambly, B.M., Ledger, S., Søjmark, A.: A McKean-Vlasov Equation with Positive Feedback and Blow-ups. Preprint (2018). Available at arXiv:1801.07703.

[37] Hellwig, M. F.: Systemic Risk in the Financial Sector: An Analysis of the SubprimeMortgage Financial Crisis. DE Economist 157, 129-207 (2009)

[38] Inglis, J., Talay, D.: Mean-Field Limit of a Stochastic Particle System Smoothly Interacting Through Threshold Hitting-Times and Applications to Neural Networks with Dendritic Component. SIAM J. Math. Anal. 47, 3884-3916 (2015)

[39] Jakubowski, A.: The Almost Sure Skorokhod Representation for Subsequences in Nonmetric Spaces. Theory Probab. Appl. 42, 167-175 (1998)

[40] Kallianpur, G. and Xiong, J.: Stochastic Differential Equations in Infinite Dimensional Spaces. Institute of Mathematical Statistics: Lecture notes, monograph series (1995)

[41] Lando, D., Nielsen, M.: Correlation in corporate defaults: Contagion or conditional independence? J. of Finan. Intermediation 19, 355-372 (2010)

[42] Ledger, S.: Sharp regularity near an absorbing boundary for solutions to second order SPDEs in a half-line with constant coefficients. Stoch. PDE: Anal. Comp. 2, 1-26 (2014)

[43] Ledger, S.: Skorokhod's M1 topology for distribution-valued processes. Electron. Commun. Probab. 21, 1-11 (2016)

[44] Moreno-Bote, R., Parga, N.: Response of integrate-and-fire neurons to noisy inputs filtered by synapses with arbitrary time scales: firing rate and correlations. Neural Comput. 22, 1528-1572 (2010)

[45] Nadtochiy, S., Shkolnikov, M.: Particle systems with singular interaction through hitting times: application in systemic risk modeling. Preprint (2017). Available at arXiv:1705.00691, 
[46] Ostojic, S., Brunel, N., Hakim, V.: Synchronization properties of networks of electrically coupled neurons in the presence of noise and heterogeneities. J. Comput. Neurosci. 26, 369-392 (2009)

[47] Pedersen, L.H.: When Everyone Runs for the Exit. Int. J. Central Banking 5, 177-199 (2009)

[48] Schwartz, L.: Radon Measures on Arbitrary Topological Spaces and Cylindrical Measures. Tata Institute of Fundamental Research, Oxford University Press, London (1973)

[49] Sowers, R., Spiliopoulos, K.: Default clustering in large pools: Large deviations. SIAM J. Fin. Math. 6, 86-116 (2015)

[50] Spiliopoulos, K.: Systemic Risk and Default Clustering for Large Financial Systems. In: Friz, P., Gatheral, J., Gulisashvili, A., Jacqier, A., Teichmann, J. (eds.) Large Deviations and Asymptotic Methods in Finance, 529-557. Springer (2015)

[51] Whitt, W.: Stochastic-Process Limits: An Introduction to Stochastic-Process Limits and Their Application to Queues. Springer (2002) 